\title{
KOSZUL COMPLEXES, HARMONIC OSCILLATORS, AND THE TODD CLASS
}

\author{
JEAN-MICHEL BISMUT
}

Dedicated to Professor M. F. Atiyah on his 60th birthday

\section{CONTENTS}

\section{0 . Introduction}

1. The Hodge theory of the resolution of a point
(a) The resolution of a point in a complex vector space
(b) The Laplacian associated with the operator $\bar{\partial}+\sqrt{-1} i_{y}$
(c) Description of the kernel of the Laplacian
(d) The cohomology of the complex $\left(\Gamma^{0}, \bar{\partial}+\sqrt{-1} i_{y}\right)$

2. The Hodge theory on the fibers of a holomorphic vector bundle
(a) A double complex on a vector bundle
(b) An enlarged double complex

3. The Levi-Civita superconnection of an acyclic chain complex
(a) A family of Dirac operators on $M$
(b) The Levi-Civita superconnection of $M$
(c) The Levi-Civita superconnection of the double complex
(d) Bianchi's identities for the superconnection $\mathscr{B}_{u}$

4. The generalized supertraces of the superconnection heat kernels
(a) The heat kernel associated with $\exp \left(-s \mathscr{B}_{u}^{2}+s b N_{H}\right)$
(b) The decay at infinity on $N_{R}$ of $P_{s}^{x, u, b}\left(Y, Y^{\prime}\right)$
(c) Translation invariance of the supertrace of the heat kernel
(d) A generalized supertrace
(e) A double transgression formula for generalized supertraces
(f) The asymptotics of the generalized supertrace as $u \rightarrow 0$
(g) A modified version of the superconnection $\mathscr{B}_{u}$

5. An extension of a formula of Mathai-Quillen: the time-dependent case

Received by the editors April 5, 1989.

1980 Mathematics Subject Classification (1985 Revision). Primary 32L10, 57R20.

Key words and phrases. Sheaves and cohomology of sections of holomorphic vector bundles, characteristic classes and numbers. 
6. A one-parameter deformation of the $\hat{A}$ Hirzebruch polynomial

(a) The function $\varphi(u, x)$

(b) The multiplicative genus $\Psi(u, \cdot)$

(c) A computation of certain finite-dimensional supertraces

(d) A computation of the trace of certain harmonic oscillators with a magnetic field

7. An explicit evaluation of the generalized supertraces

(a) A path integral formula for $\operatorname{Tr}_{s}\left[P_{1}^{x, u, b}(Y, Y)\right]$

(b) Generalized supertraces and infinite determinants

(c) The asymptotics of the generalized supertraces as $u \rightarrow+\infty$

8. Generalized supertraces, analytic torsion, and the Todd genus

(a) A construction of the generalized analytic torsion forms

(b) An evaluation of the analytic torsion forms

9. Equivariant intersection theory and the harmonic oscillator

(a) Equivariant exact sequences and differential forms

(b) The asymptotics of the forms $\eta(u, b)$

(c) An evaluation of the forms $\eta(u, b)$

(d) Fourier series and generalized analytic torsion forms

Appendix by J.-M. Bismut and C. Soulé

\section{INTRODUCTION}

The purpose of this paper is to construct and identify certain characteristic classes naturally associated with an acyclic chain complex of holomorphic Hermitian vector bundles

$$
0 \rightarrow L \underset{i}{\rightarrow} M \underset{j}{\rightarrow} N \rightarrow 0
$$

on a complex manifold $B$.

More precisely, let $g^{M}$ be a Hermitian metric on $M$, and let $g^{L}$ and $g^{N}$ be the Hermitian metrics induced by $g^{M}$ on $L$ and $N$. For $u>0$, we consider the family of operators $\bar{\partial}+\sqrt{-u} i_{j(y)}$ acting on smooth sections of $\Lambda\left(\bar{M}^{*}\right) \hat{\otimes} \Lambda\left(N^{*}\right)$ along fibers of $M_{R}$, naturally associated with the fiberwise resolution of the sheaf $i_{*} \mathscr{O}_{L}$ by the Koszul complex of the normal bundle $N$. We then construct the corresponding Hodge theory along the fibers of $M_{R}$, and we obtain a family of elliptic operators $\mathscr{L}_{u}=\bar{\partial}+\sqrt{-u} i_{j(y)}+\bar{\partial}^{*}-\sqrt{-u} i_{j(y)}^{*}$ along the fibers of $M_{R}$. We calculate the Levi-Civita superconnection $\mathscr{B}_{u}$ of the family in the sense of Bismut [B1, §3]. In fact, in [B1], the Levi-Civita superconnection of a fibration with compact fibers was essential in proving the local version of the Atiyah-Singer Index Theorem for families.

The curvature $\mathscr{B}_{u}^{2}$ of $\mathscr{B}_{u}$ in the sense of Quillen [Q1] is essentially a nontrivial coupling of a harmonic oscillator on $N_{R}$ to the Laplacian on $L_{R}$. Let $P^{x, u}\left(Y, Y^{\prime}\right)$ be the heat kernel associated with $\exp \left(-\mathscr{B}_{u}^{2}\right)$. The operator $\exp \left(-\mathscr{B}_{u}^{2}\right)$ is not fiberwise trace-class. However, we prove in Theorems 4.1 
and 4.2 that the supertrace $\operatorname{Tr}_{s}\left[P^{x, u}(Y, Y)\right]$ is fiberwise invariant by translations by elements of $L_{R}$ and decays quickly as $Y \in N_{R}$ tends to infinity. It is then natural to form a generalized supertrace

$$
\operatorname{Tr}_{s}\left[\exp \left(-\mathscr{B}_{u}^{2}\right)\right]=\int_{N_{R}} \operatorname{Tr}_{s}\left[P^{x, u}(Y, Y)\right] \frac{d v_{N}(Y)}{(2 \pi)^{\operatorname{dim} N}} .
$$

$\operatorname{Tr}_{s}\left[\exp \left(-\mathscr{B}_{u}^{2}\right)\right]$ is a smooth form on $B$. It is a sort of generalized Chern character for the family of operators $\mathscr{L}_{u}$.

Let $\operatorname{Td}\left(g^{L}\right), \operatorname{Td}\left(g^{M}\right)$, and $\operatorname{Td}\left(g^{N}\right)$ be the Chern-Weil representatives of the Todd class of $L, M$, and $N$ naturally associated with the holomorphic Hermitian connections on $L, M$, and $N$. Let $\Phi$ denote the scaling map $\alpha \in \Lambda^{\text {even }}\left(T_{R}^{*} B\right) \rightarrow(2 \pi i)^{-(\operatorname{deg} \alpha) / 2} \alpha \in \Lambda^{\text {even }}\left(T_{R}^{*} B\right)$. We then prove in Theorems 4.6, 4.8, and 7.7 that the forms $\Phi \operatorname{Tr}_{s}\left[\exp \left(-\mathscr{B}_{u}^{2}\right)\right]$ are sums of forms of type $(p, p)$ and closed, that their cohomology class does not depend on $u>0$, and that moreover

$$
\begin{aligned}
& \lim _{u \rightarrow 0} \Phi \operatorname{Tr}_{s}\left[\exp \left(-\mathscr{B}_{u}^{2}\right)\right]=\frac{\operatorname{Td}\left(g^{M}\right)}{\operatorname{Td}\left(g^{N}\right)}, \\
& \lim _{u \rightarrow \infty} \Phi \operatorname{Tr}_{s}\left[\exp \left(-\mathscr{B}_{u}^{2}\right)\right]=\operatorname{Td}\left(g^{L}\right) .
\end{aligned}
$$

The family of forms $\Phi \operatorname{Tr}_{s}\left[\exp \left(-\mathscr{B}_{u}^{2}\right)\right]$ interpolates between the form $\operatorname{Td}\left(g^{M}\right) / \operatorname{Td}\left(g^{N}\right)$ and $\operatorname{Td}\left(g^{L}\right)$, and is obtained by a construction in infinite dimensions. Quite remarkably, we prove double transgression formulas for generalized supertraces which are formally identical to similar formulas proved in Bismut-Gillet-Soulé [BGS1, Theorem 1.15; BGS2, Theorem 2.9] for finiteor infinite-dimensional ordinary supertraces. Namely, we prove in Theorem 4.6 that if $N_{H}$ is the number operator of the complex $\Lambda\left(N^{*}\right)$, then we have the identity of smooth forms on $B$

$$
\begin{aligned}
\frac{\partial}{\partial u} \operatorname{Tr}_{s}\left[\exp \left(-\mathscr{B}_{u}^{2}\right)\right] & =\frac{\bar{\partial} \partial}{u} \frac{\partial}{\partial b}\left[\operatorname{Tr}_{s}\left[\exp \left(-\mathscr{B}_{u}^{2}+b N_{H}\right)\right]\right]_{b=0} \\
& =\frac{\bar{\partial} \partial}{u} \operatorname{Tr}_{s}\left[N_{H} \exp \left(-\mathscr{B}_{u}^{2}\right)\right] .
\end{aligned}
$$

It is then possible to integrate equation $(0.4)$ by a zeta function technique inspired from Ray-Singer [RS] and very similar to [BGS1, Definition 1.16 and BGS2, Definition 2.19]. We thus obtain in $\S 8$ generalized analytic torsion forms $\mathbb{B}\left(L, M, g^{M}\right)$ on $B$ which, by Theorem 8.3 , verify the equation

$$
\frac{\bar{\partial} \partial}{2 i \pi} \mathbb{B}\left(L, M, g^{M}\right)=\operatorname{Td}\left(g^{L}\right)-\frac{\operatorname{Td}\left(g^{M}\right)}{\operatorname{Td}\left(g^{N}\right)} .
$$

The main purpose of this paper is to calculate the form $\mathbb{B}\left(L, M, g^{M}\right)$ modulo $\partial$ and $\bar{\partial}$ coboundaries. In fact by a construction of Bott-Chern [BoC], Donaldson [D], and Bismut-Gillet-Soulé [BGS1, §1f)], we know how to solve in a natural way the equation

$$
\frac{\bar{\partial} \partial}{2 i \pi} \widetilde{\mathrm{Td}}\left(L, M, g^{M}\right)=\operatorname{Td}\left(g^{M}\right)-\operatorname{Td}\left(g^{L}\right) \operatorname{Td}\left(g^{N}\right)
$$


so that if the complex $(0.1)$ splits, $\widetilde{T d}\left(L, M, g^{M}\right)=0$. The construction of $\widetilde{\mathrm{Td}}\left(L, M, g^{M}\right)$ is essentially finite dimensional. In view of $(0.5)$ and $(0.6)$, the question arises to calculate $\mathbb{B}\left(L, M, g^{M}\right)+\mathrm{Td}^{-1}\left(g^{N}\right) \widetilde{\mathrm{Td}}\left(L, M, g^{M}\right)$.

We owe a word of explanation to the reader to describe the fundamental importance of this calculation in our strategy to understand the behavior of the Quillen metrics on the determinant of the cohomology of a complex vector bundle by an immersion of complex manifolds. In fact if $i: Y \rightarrow X$ is an immersion of compact complex manifolds, and if $\xi \rightarrow i_{*} \eta \rightarrow 0$ is a resolution of the sheaf of sections of a holomorphic vector bundle $\eta$ on $Y$ by a complex of holomorphic vector bundles $\xi$ on $X$, then the determinants of the cohomology $\lambda(\xi)$ and $\lambda(\eta)$ are canonically isomorphic.

If metrics are introduced on $X, Y, \xi$, and $\eta$, then $\lambda(\xi)$ and $\lambda(\eta)$ carry natural metrics called Quillen metrics [Q2, BGS3]. It is then natural to compare these metrics. In our sketched proof of the comparison formula, the form $\mathbb{B}\left(T Y, T X_{\mid Y}, g^{T X_{\mid Y}}\right)$ (where $g^{T X}$ is the metric on $T X$ ) appears naturally. This is why its explicit calculation (modulo irrelevant $\partial$ and $\bar{\partial}$ coboundaries) is so important. The general program of describing the behavior of Quillen metrics by immersions has recently come to a successful conclusion in a joint work by Lebeau and ourselves [BL1, BL2]. The results contained in the present paper play an important role in establishing the final formula of [BL1, BL2].

We now briefly explain the main results contained in this paper. We introduce the function of $u \geq 0$ and $x \in \mathbb{C}$ :

$$
\varphi(u, x)=\frac{4}{u} \sinh \left(\frac{x+\sqrt{x^{2}+4 u}}{4}\right) \sinh \left(\frac{-x+\sqrt{x^{2}+4 u}}{4}\right) .
$$

The function $\varphi(u, x)$ has two fundamental properties. A first property is that

$$
\varphi(0, x)=\frac{\sinh (x / 2)}{x / 2} .
$$

$\varphi(u, x)$ is then a one-parameter deformation of the inverse of the Hirzebruch polynomial $\widehat{A}(x)$. A second property of $\varphi(u, x)$ is that, as shown in Theorem 6.2 ,

$$
\varphi(u, x)=\prod_{k=1}^{+\infty}\left(1+\frac{i x}{2 k \pi}+\frac{u}{4 k^{2} \pi^{2}}\right)\left(1-\frac{i x}{2 k \pi}+\frac{u}{4 k^{2} \pi^{2}}\right) .
$$

Let $C(s, x)$ be the Mellin transform of $-\frac{\partial \varphi}{\partial x}(u, x) / \varphi(u, x)$, i.e., for $|x|<2 \pi$ and $0<\operatorname{Re}(s)<\frac{1}{2}$, set

$$
C(s, x)=\frac{1}{\Gamma(s)} \int_{0}^{+\infty} u^{s-1}\left(\frac{-\frac{\partial \varphi}{\partial x}(u, x)}{\varphi(u, x)}\right) d u .
$$

Then $C$ extends holomorphically at $s=0$. Set

$$
D(x)=\frac{\partial C}{\partial s}(0, x) \text {. }
$$

Let $D(N)$ be the corresponding additive class associated with the vector bundle $N$, and let $\operatorname{Td}(L)$ be the Todd class of $L$. Then $\operatorname{Td}(L) D(N)$ is a well-defined class of sums of $(p, p)$ forms modulo $\partial$ and $\bar{\partial}$ coboundaries. 
We then prove in Theorem 8.5 the following identity modulo $\partial$ and $\bar{\partial}$ coboundaries:

$$
\mathbb{B}\left(L, M, g^{M}\right)=-\operatorname{Td}^{-1}\left(g^{N}\right) \widetilde{\operatorname{Td}}\left(L, M, g^{M}\right)+\operatorname{Td}(L) D(N) .
$$

Let $\zeta(s)$ be the Riemann zeta function. In a joint appendix with C. Soulé, we calculate the function $D(x)$ in the form

$$
D(x)=\sum_{\substack{n \geq 1 \\ \text { odd }}}\left(\Gamma^{\prime}(1)+\sum_{j=1}^{n} \frac{1}{j}+2 \frac{\zeta^{\prime}(-n)}{\zeta(-n)}\right) \zeta(-n) \frac{x^{n}}{n !}, \quad|x|<2 \pi .
$$

(0.12) is an extremely interesting formula. In fact in [GS], Gillet and Soulé introduced the power series

$$
R(x)=\sum_{\substack{n \geq 1 \\ \text { odd }}}\left(\sum_{1}^{n} \frac{1}{j}+2 \frac{\zeta^{\prime}(-n)}{\zeta(-n)}\right) \zeta(-n) \frac{x^{n}}{n !}
$$

by a complicated calculation (with D. Zagier) of the Ray-Singer analytic torsion of $\mathbb{P}^{n}(\mathbb{C})$. Gillet and Soule conjectured that the additive genus $R(x)$ should play an important role in an arithmetic version of a theorem of Riemann-RochGrothendieck with metrics. It is remarkable that an entirely different construction from [GS] produces a closely related genus $D(x)$.

Finally in Theorem 9.13, we show that if $p_{t}(x)$ is the standard heat kernel on $S_{1}$, then

$$
D(x)=-\int_{0}^{+\infty}(\log r) \frac{\partial p_{r}}{\partial x}(r x) d r .
$$

We now say just a few words about our techniques. We use Quillen's superconnections [Q1] in an infinite-dimensional context in a rather different way from our work [B1] on the families Index Theorem. Harmonic oscillators play a key role in the whole paper. Local index cancellation techniques [ABP, B1, B8, $\mathrm{Ge}$ ] also appear in the course of the proofs. To establish our main results on the forms $\operatorname{Tr}_{s}\left[\exp \left(-\mathscr{B}_{u}^{2}\right)\right]$, we calculate these forms in a completely explicit way in Theorem 7.3 as the ratio of determinants of differential operators of order two on the circle. This in turn uses Brownian motion and also some rigorous version of fermionic integration which is developed in $\S 5$.

As shown in $\S 9$, the computations in this paper can also be understood from the point of view of infinite-dimensional intersection theory. We show in $\S 9$ (d) that $\mathbb{B}\left(L, M, g^{M}\right)$ is simply the integral along the fiber of an infinitedimensional Euler-Green current in the sense of Bismut-Gillet-Soule [BGS5, $\S 3]$. In fact, we first calculated the form $\mathbb{B}\left(L, M, g^{M}\right)$ in the path integral formalism, obtained formula (0.11), calculated formula $(0.12)$ with C. Soule, and then reconstructed the form $\mathbb{B}\left(L, M, g^{M}\right)$ in the superconnection formalism.

Needless to say, it is of fundamental importance that the form $\mathbb{B}\left(L, M, g^{M}\right)$ can be constructed from an analytic point of view-this is the superconnection construction-and from a geometric point of view-which is implicit in the path integral formalism. 
This paper contains nine sections and an appendix. In $\S 1$, we describe the Hodge theory of the resolution of a point in a Hermitian complex vector space. In $\S 2$, we consider the Hodge theory for the resolution of a point in the fibers of a holomorphic Hermitian vector bundle. In $\S 3$, we introduce the superconnection $\mathscr{B}_{u}$, and we prove nontrivial Bianchi identities on the curvature $\mathscr{B}_{u}{ }_{u}$. In $\S 4$, we construct the heat kernels $\exp \left(-\mathscr{B}_{u}^{2}\right)$ and the generalized supertraces $\operatorname{Tr}_{s}\left[\exp \left(-\mathscr{B}_{u}^{2}\right)\right]$. We prove the double transgression formula (0.4), and the first identity in (0.3). In $\S 5$, we prove a time dependent version of an identity of Mathai-Quillen [MQ] for finite-dimensional supertraces. In $\S 6$, we introduce the function $\varphi(u, x)$ and describe its main properties. In $\S 7$, we calculate explicitly the forms $\operatorname{Tr}_{s}\left[\exp \left(-\mathscr{B}_{u}^{2}+b N_{H}\right)\right]$ and we prove the second identity in (0.3). In $\S 8$, we construct the form $\mathbb{B}\left(L, M, g^{M}\right)$ and we prove (0.5) and (0.11). In $\S 9$, we develop a formalism for the equivariant intersection theory for holomorphic Hermitian vector bundles, and we relate the corresponding results with the results of $\S \S 3-8$. We also prove formula (0.14). Finally, in a joint appendix with C. Soulé, we prove formula (0.12).

We assume the reader has some familarity with superconnections. We refer to Quillen [A1] and to the present author [B1, B5] for an introduction to the subject. Let us just say that if $A$ is a $\mathbb{Z}_{2}$ graded algebra, and if $f, f^{\prime} \in A$, then the supercommutator $\left[f, f^{\prime}\right]$ is defined by

$$
\left[f, f^{\prime}\right]=f f^{\prime}-(-1)^{\operatorname{deg}(f) \operatorname{deg}\left(f^{\prime}\right)} f^{\prime} f .
$$

If $E=E_{+} \oplus E_{-}$is a $\mathbb{Z}_{2}$ graded vector space, then End $E$ is naturally $\mathbb{Z}_{2}$ graded, with the even (resp. odd) elements of End $E$ preserving (resp. exchanging) $E_{+}$ and $E_{-}$. If $\tau$ is the involution defining the grading, the supertrace $\operatorname{Tr}_{s}(f)$ of $f \in$ End $E$ is by definition

$$
\operatorname{Tr}_{s}(f)=\operatorname{Tr}(\tau f) \text {. }
$$

If $V$ is another vector space, if $\omega \in \Lambda\left(V^{*}\right)$, and if $f \in \operatorname{End} E$, then set

$$
\operatorname{Tr}_{s}(\omega f)=\omega \operatorname{Tr}_{s}(f) \text {. }
$$

We then extend by linearity $\operatorname{Tr}_{s}$ into a linear map from $\Lambda\left(V^{*}\right) \hat{\otimes}$ End $E$ into $\Lambda\left(V^{*}\right)$. Throughout the paper, it is essential to take into account the natural commutation rules on graded algebras to get the right signs.

In the sequel, if $Y$ is a Brownian motion, $d Y$ and $\delta Y$ denote respectively its Stratonovitch and Itô differentials [IkW, Chapters II and III]. For an introduction to the probabilistic techniques used in this paper, we refer to our survey [B6].

The results contained in this paper were announced in [B12].

\section{THE Hodge theORY OF THE RESOlUtion OF A POINT}

Let $V$ be a complex Hermitian vector space, and let $\left(\Lambda V^{*}, i_{y}\right)$ be the Koszul complex of $V$. In this section, we study the Hodge theory associated with the double complex $\left(\Gamma\left(\Lambda\left(\bar{V}^{*}\right) \hat{\otimes} \Lambda\left(V^{*}\right)\right), \bar{\partial}+\sqrt{-1} i_{y}\right)$. The corresponding Hodge Laplacian is a harmonic oscillator. The form $\beta$ on $V_{R}$, which is a Hodge 
representative of the one-dimensional cohomology of the double complex, is also described.

This section is organized as follows. In (a), we construct the double complex $\left(\Gamma\left(\Lambda\left(\bar{V}^{*}\right) \hat{\otimes} \Lambda\left(V^{*}\right)\right), \bar{\partial}+\sqrt{-1} i_{y}\right)$. In (b), we calculate the corresponding Hodge Laplacian. In (c), we calculate the kernel of the Laplacian. Finally, in (d), we relate our calculations in $L_{2}$ cohomology with the standard sheaf theoretic results on the resolution of a point.

Although they are elementary, the results of this section will play an essential role in the sequel. Also let us point out that they are formally related to similar calculations by Witten [W] in de Rham cohomology.

(a) The resolution of a point in a complex vector space. Let $V_{R}$ be a real vector space of even dimension $2 n$. Let $\mathbf{J}$ be a complex structure on $V_{K}$, i.e., a linear map in $\operatorname{End}\left(V_{R}\right)$ which is such that $\mathrm{J}^{2}=-1$. Let $V$ and $\bar{V}$ be the eigenspaces in $V_{R} \otimes_{R} \mathbb{C}$ associated with the eigenvalues of $\mathbf{J} \sqrt{-1}$ and $-\sqrt{-1}$. As indicated by the notation, $\bar{V}$ is conjugate to $V$.

Let $V_{R}^{*}$ be the set of $\mathbb{R}$ linear forms on $V_{R}$, and let $V^{*}$ and $\bar{V}^{*}$ be the set of $\mathbb{C}$ linear forms on $V$ and $\bar{V}$.

The exterior algebras $\Lambda\left(V^{*}\right)$ and $\Lambda\left(\bar{V}^{*}\right)$ are naturally $\mathbb{Z}$ graded. Let $N_{H}$ and $N_{V}$ be the linear maps defining the $\mathbb{Z}$ grading on $\Lambda\left(V^{*}\right)$ and $\Lambda\left(\bar{V}^{*}\right)$, so that $N_{H}$ and $N_{V}$ act by multiplication by $p$ on $\Lambda^{p}\left(V^{*}\right)$ and $\Lambda^{p}\left(\bar{V}^{*}\right)$ respectively. Note the identity

$$
\Lambda\left(V_{R}^{*}\right) \otimes_{R} \mathbb{C}=\Lambda\left(\bar{V}^{*}\right) \hat{\otimes} \Lambda\left(V^{*}\right) .
$$

The algebra $\Lambda\left(V_{R}^{*}\right) \otimes_{R} \mathbb{C}$ possesses a natural $\mathbb{Z}$ grading given by the operator $N_{V} \otimes 1+1 \otimes N_{H}$. Here we will instead use the grading of $\Lambda\left(V_{R}^{*}\right) \otimes_{R} \mathbb{C}$ by the operator $N_{V} \otimes 1-1 \otimes N_{H}$ (which we will also write in the form $N_{V}-N_{H}$ ).

Let $\Gamma$ be the set of smooth sections of $\Lambda\left(\bar{V}^{*}\right) \hat{\otimes} \Lambda\left(V^{*}\right)$ on $V_{R}$. We also grade $\Gamma$ by the operator $N_{V}-N_{H}$. Let $\bar{\partial}$ be the Dolbeault operator acting on $\Gamma$. For $y \in V$, the interior multiplication operator $i_{y}$ acts on $\Lambda\left(\bar{V}^{*}\right) \hat{\otimes} \Lambda\left(V^{*}\right)$. If $y \in V$, set $Y=y+\bar{y} \in V_{R}$. Then the operator $i_{y}$ acts naturally on $\Gamma$, with the convention that if $f \in \Gamma$, then $\left(i_{y} f\right)(Y)=i_{y}(f(Y))$. Both operators $\bar{\partial}$ and $i_{y}$ now act on $\Gamma$ and increase the total degree of $\Gamma$ by one. Moreover,

$$
\left(\bar{\partial}+\sqrt{-1} i_{y}\right)^{2}=0 .
$$

Let $i$ be the embedding of complex manifolds: $\{0\} \rightarrow V$.

Note that the $\bar{\partial}$ cohomology of $\{0\}$ is concentrated in degree zero, i.e., $H^{q}\{0\}=\mathbb{C}$ if $q=0$, and $H^{q}\{0\}=0$ if $q>0$. The Dolbeault complex of $\{0\}$ is here simply $\Omega^{(0,0)}\{0\}=\mathbb{C} \underset{\bar{\partial}^{\{0\}}}{\rightarrow} 0$, i.e., $\bar{\partial}^{\{0\}}$ is the zero map. We give the degree zero to $\Omega^{(0,0)}\{0\}$.

If $\omega \in \Lambda\left(\bar{V}^{*}\right) \widehat{\otimes} \Lambda\left(V^{*}\right)$, let $\omega^{0}$ denote the component of $\omega$ in $\Lambda\left(\bar{V}^{*}\right) \otimes \mathbb{C}=$ $\Lambda\left(\bar{V}^{*}\right)$. Let $r$ denote the map $\omega \in \Gamma \rightarrow i^{*} \omega^{0} \in \mathbb{C}=\Omega^{(0,0)}\{0\}$. Note that if $\omega \in \Lambda^{q}\left(\bar{V}^{*}\right) \hat{\otimes} \Lambda^{p}\left(V^{*}\right), p, q \in \mathbb{N}, p+q>0$, then $r \omega=0$. In particular if $\omega$ has total degree $k$ in $\Gamma, k \neq 0$, then $r \omega=0$. 
Proposition 1.1. The map

$$
r:\left(\Gamma, \bar{\partial}+\sqrt{-1} i_{y}\right) \rightarrow\left(\Omega^{(0,0)}, \bar{\partial}^{\{0\}}\right)
$$

is a quasi-isomorphism of $\mathbb{Z}$ graded complexes.

Proof. Clearly if $\omega \in \Gamma$, then

$$
r\left(\bar{\partial}+\sqrt{-1} i_{y}\right) \omega=0
$$

and so $r$ is a map of complexes. The complex $\left(\Gamma, \bar{\partial}+\sqrt{-1} i_{y}\right)$ is a double complex. To calculate its cohomology, we use a spectral sequence. Let $\mathscr{O}\left(\Lambda V^{*}\right)$ be the set of holomorphic sections of $\Lambda V^{*}$ on $V_{R}$. By the Poincare lemma [GH, p. 25], we know that

$$
H^{q}(\Gamma, \bar{\partial})= \begin{cases}\mathscr{O}\left(\Lambda V^{*}\right) & \text { if } q=0 \\ 0 & \text { if } q>0\end{cases}
$$

The complex $\left(\mathscr{O}\left(\Lambda V^{*}\right), i_{y}\right)$ is exactly the Koszul complex of $V$. Let $r_{0}$ be the restriction map

$$
r_{0}: f \in \mathscr{O} \rightarrow f(0) \in \mathbb{C} .
$$

By [GH, p. 687], we have the exact sequence

$$
0 \rightarrow \mathscr{O}\left(\Lambda^{n} V^{*}\right) \underset{i_{y}}{\rightarrow} \cdots \underset{i_{y}}{\rightarrow} \mathscr{O}\left(V^{*}\right) \underset{i_{y}}{\rightarrow} \underset{r_{0}}{\rightarrow} \mathbb{C} \rightarrow 0 .
$$

Equivalently,

$$
H_{q}\left(\mathscr{O}\left(\Lambda V^{*}\right), i_{y}\right)= \begin{cases}\mathbb{C} & \text { if } q=0 \\ \{0\} & \text { if } q \neq 0\end{cases}
$$

Also the map $r_{0}$ induces the isomorphism $H^{0}\left(\mathscr{O}\left(\Lambda V^{*}\right), i_{y}\right) \cong \mathbb{C}$. Moreover if $\omega \in \mathscr{O}$, then $r \omega=r_{0} \omega$. The proposition follows.

(b) The Laplacian associated with the operator $\bar{\partial}+\sqrt{-1} i_{y}$. Let $\langle$,$\rangle be a J-$ invariant scalar product on $V_{R}$. Then $y, y^{\prime} \in V \rightarrow\left\langle y, \bar{y}^{\prime}\right\rangle \in \mathbb{C}$ is a Hermitian product on $V$. For $y \in V$, set $|y|=\langle y, \bar{y}\rangle^{1 / 2}$. To $y \in V$, we associate $Y=y+\bar{y} \in V_{R}$. Note that if $|Y|^{2}=\langle Y, Y\rangle$, then

$$
|Y|^{2}=2|y|^{2} \text {. }
$$

Let $\theta$ be the Kähler form on $V_{R}$, i.e., the two-form

$$
X, Y \rightarrow \theta(X, Y)=\langle X, \mathrm{~J} Y\rangle .
$$

Then $\theta$ is a form of complex type $(1,1)$ and has total degree zero in $\Lambda\left(\bar{V}^{*}\right) \hat{\otimes}$ $\Lambda\left(V^{*}\right)$.

Let $\left(\partial / \partial a^{1}, \partial / \partial b^{1}, \ldots, \partial / \partial a^{n}, \partial / \partial b^{n}\right)$ be an orthonormal base of $V_{R}$, with $\partial / \partial b^{j}=\mathbf{J} \partial / \partial a^{j}, 1 \leq j \leq n$. Let $\left(d a^{1}, d b^{1}, \ldots, d a^{n}, d b^{n}\right)$ be the corresponding dual base in $V_{R}^{*}$. Set

$$
\begin{array}{rlrl}
\frac{\partial}{\partial y^{j}}= & \frac{1}{2}\left(\frac{\partial}{\partial a^{j}}-\sqrt{-1} \frac{\partial}{\partial b^{j}}\right), & & \frac{\partial}{\partial \bar{y}^{j}}=\frac{1}{2}\left(\frac{\partial}{\partial a^{j}}+\sqrt{-1} \frac{\partial}{\partial b^{j}}\right), \\
d y^{j}=d a^{j}+\sqrt{-1} d b^{j}, & d \bar{y}^{j}=d a^{j}-\sqrt{-1} d b^{j} .
\end{array}
$$


Then $\theta$ is given by

$$
\theta=-\frac{\sqrt{-1}}{2} \sum_{1}^{n} d y^{j} \wedge d \bar{y}^{j}
$$

Note that if $y=\sum_{1}^{n} y^{i} \partial / \partial y^{i}$, then

$$
|y|^{2}=\frac{1}{2} \sum_{1}^{n}\left|y^{i}\right|^{2} .
$$

Let $\Gamma^{0}$ be the set of square-integrable sections of $\Lambda\left(\bar{V}^{*}\right) \hat{\otimes} \Lambda\left(V^{*}\right)$ on $V_{R}$. By [GH, p. 82], the star operator $*$ acts naturally on $\Lambda\left(V_{R}^{*}\right) \otimes_{R} \mathbb{C}=\Lambda\left(\bar{V}^{*}\right) \hat{\otimes} \Lambda\left(V^{*}\right)$. We equip $\Gamma^{0}$ with the Hermitian product

$$
f, g \in \Gamma^{0} \rightarrow\langle f, g\rangle=\left(\frac{1}{2 \pi}\right)^{n} \int_{V_{R}} f \wedge * g .
$$

$\Lambda\left(V_{R}^{*}\right) \otimes_{R} \mathbb{C}$ inherits a Hermitian product $\langle$,$\rangle from the scalar product of V_{R}$. If $d v=(-\theta)^{n} / n$ ! is the volume form on $V_{R}$, then for $f, g \in \Gamma^{0}$

$$
\langle f, g\rangle=\left(\frac{1}{2 \pi}\right)^{n} \int_{V_{R}}\langle f, g\rangle d v .
$$

Let $\bar{\partial}^{*}$ be the formal adjoint of $\bar{\partial}$, with respect to the Hermitian product (1.11), and let $i_{y}^{*}$ be the adjoint of the operator $i_{y}$. Then the operator $\bar{\partial}^{*}-$ $\sqrt{-1} i{ }_{y}^{*}$ also acts on $\Gamma$, and decreases the degree in $\Gamma$ by 1 .

We first calculate the operators $\bar{\partial}+\sqrt{-1} i i_{y}$ and $\bar{\partial}^{*}-\sqrt{-1} i_{y}^{*}$ in the coordinates $y^{1}, \ldots, y^{n}$.

Proposition 1.2. The following identities hold:

$$
\begin{gathered}
\bar{\partial}+\sqrt{-1} i_{y}=\sum_{1}^{n} d \bar{y}^{j} \wedge \frac{\partial}{\partial \bar{y}^{j}}+\sqrt{-1} \sum_{1}^{n} y^{j} i_{\partial / \partial y^{j}}, \\
\bar{\partial}^{*}-\sqrt{-1} i_{y}^{*}=-2 \sum_{1}^{n} i_{\partial / \partial \bar{y}^{j}} \frac{\partial}{\partial y^{j}}-\frac{\sqrt{-1}}{2} \sum_{1}^{n} \bar{y}^{j} d y^{j} \wedge .
\end{gathered}
$$

Proof. The first line in (1.13) is obvious. Note that

$$
\left|d y^{i}\right|^{2}=2 ; \quad\left|\frac{\partial}{\partial y^{i}}\right|^{2}=\frac{1}{2} .
$$

The second line in (1.13) now follows from (1.14).

Let $c\left(V_{R}\right)$ be the Clifford algebra in $V_{R}$. Then $\Lambda\left(\bar{V}^{*}\right)$ and $\Lambda\left(V^{*}\right)$ are $c\left(V_{R}\right)$ Clifford modules. In fact if $X \in V$, let $X^{*} \in \bar{V}^{*}$ be the element which corresponds to $X$ by the Hermitian product of $V$. Then if $X \in V$ and $X^{\prime} \in \bar{V}$, set

$$
c(X)=\sqrt{2} X^{*} \wedge ; \quad c\left(X^{\prime}\right)=-\sqrt{2} i_{X^{\prime}}
$$


We then extend by $\mathbb{C}$ linearity the map $X \in V_{R} \otimes_{R} \mathbb{C} \rightarrow c(X)$. One verifies easily that if $X, X^{\prime} \in V_{R} \otimes_{R} \mathbb{C}$, then

$$
c(X) c\left(X^{\prime}\right)+c\left(X^{\prime}\right) c(X)=-2\left\langle X, X^{\prime}\right\rangle .
$$

Similarly if $X^{\prime} \in \bar{V}$, let $X^{\prime *}$ be the corresponding element in $V^{*}$. If $X \in V$ and $X^{\prime} \in \bar{V}$, set

$$
\hat{c}(X)=-\sqrt{2} \sqrt{-1} i_{X^{\prime}} ; \quad \hat{c}\left(X^{\prime}\right)=-\sqrt{2} \sqrt{-1} X^{\prime *} \wedge .
$$

We extend $\hat{c}$ to the whole $V_{R} \otimes_{R} \mathbb{C}$ by $\mathbb{C}$ linearity. Again if $X, X^{\prime} \in V_{R} \otimes_{R} \mathbb{C}$, then

$$
\hat{c}(X) \hat{c}\left(X^{\prime}\right)+\hat{c}\left(X^{\prime}\right) \hat{c}(X)=-2\left\langle X, X^{\prime}\right\rangle .
$$

Of course, we make the operators $c(X)$ and $\hat{c}(X)$ act as odd operators on $\Lambda\left(V_{R}^{*}\right) \otimes_{R} \mathbb{C}=\Lambda\left(\bar{V}^{*}\right) \hat{\otimes} \Lambda\left(V^{*}\right)$. If $X, X^{\prime} \in V_{R} \otimes_{R} \mathbb{C}$, we then also have

$$
c(X) \hat{c}\left(X^{\prime}\right)+\hat{c}\left(X^{\prime}\right) c(X)=0 .
$$

Let $L$ be the operator

$$
\alpha \in \Lambda\left(V_{R}^{*}\right) \otimes_{R} \mathbb{C} \rightarrow L \alpha=\theta \wedge \alpha .
$$

Let $\Lambda$ be the adjoint of $L$. Using the notations in (1.13), we find that

$$
L=\frac{-\sqrt{-1}}{2} \sum_{1}^{n} d y^{j} \wedge d \bar{y}^{j} \wedge, \quad \Lambda=2 \sqrt{-1} \sum_{1}^{n} i_{\partial / \partial \bar{y}^{j}} i_{\partial / \partial y^{j}}
$$

Definition 1.3. $S$ denotes the operator

$$
S=-(L+\Lambda) .
$$

Let $e_{1}, \ldots, e_{2 n}$ be a real orthonormal base of $V_{R}$. Let $\Delta$ be the standard Laplacian on $V_{R}$. If $f \in V_{R}$, then $\nabla_{f}$ denotes the differentiation operator along the vector $f$. We now calculate the Laplacian associated with the Hermitian chain complex $\left(\Gamma^{0}, \bar{\partial}+\sqrt{-1} i_{y}\right)$.

Proposition 1.4. The following identities hold:

$$
\begin{gathered}
\left(\bar{\partial}+\sqrt{-1} i_{y}\right)^{2}=0 ; \quad\left(\bar{\partial}^{*}-\sqrt{-1} i_{y}^{*}\right)^{2}=0, \\
\bar{\partial}+\sqrt{-1} i_{y}+\bar{\partial}^{*}-\sqrt{-1} i_{y}^{*}=\frac{1}{\sqrt{2}}\left(\sum_{1}^{2 n} c\left(e_{i}\right) \nabla_{e_{i}}+\sqrt{-1} \hat{c}(\mathbf{J} Y)\right), \\
S=\frac{\sqrt{-1}}{2} \sum_{1}^{2 n} c\left(e_{i}\right) \hat{c}\left(\mathbf{J} e_{i}\right), \\
\left(\bar{\partial}+\sqrt{-1} i_{y}+\bar{\partial}^{*}-\sqrt{-1} i_{y}^{*}\right)^{2}=-\frac{\Delta}{2}+\frac{|Y|^{2}}{2}+S .
\end{gathered}
$$

Proof. The first line in (1.23) is obvious. The second line in (1.23) is a consequence of Proposition 1.2 and of the fact that $Y=y+\bar{y}$. Let $w_{1}, \ldots, w_{n}$ be 
an orthonormal base in $V$, let $w^{1}, \ldots, w^{n}$ be the dual base in $V^{*}$, and let $\bar{w}_{1}, \ldots, \bar{w}_{n}$ and $\bar{w}^{1}, \ldots, \bar{w}^{n}$ be the conjugate bases in $\bar{V}$ and $\bar{V}^{*}$. Then

$$
\begin{aligned}
\frac{\sqrt{-1}}{2} \sum_{1}^{2 n} c\left(e_{j}\right) \hat{c}\left(\mathbf{J} e_{j}\right) & =\frac{1}{2} \sum_{1}^{n}\left(c\left(w_{j}\right) \hat{c}\left(\bar{w}_{j}\right)-c\left(\bar{w}_{j}\right) \hat{c}\left(w_{j}\right)\right) \\
& =\sqrt{-1} \sum_{1}^{n}\left(-\bar{w}^{j} \wedge w^{j} \wedge+i_{w^{j}} i_{\bar{w}^{j}}\right)=-L-\Lambda .
\end{aligned}
$$

The third line in (1.23) follows. The fourth line in (1.23) is an easy consequence of lines two and three in (1.23).

(c) Description of the kernel of the Laplacian. We now study the kernel of the operator $\left(\bar{\partial}+\sqrt{-1} i_{y}+\bar{\partial}^{*}-\sqrt{-1} i_{y}^{*}\right)^{2}$.

The algebra $\Lambda^{\text {even }}\left(V_{R}\right) \otimes_{R} \mathbb{C}$ is commutative, and so the exponential maps $\Lambda^{\text {even }}\left(V_{R}\right) \otimes_{R} \mathbb{C}$ into itself. In particular, $\exp (\theta) \in \Lambda^{\text {even }}\left(V_{R}\right) \otimes_{R} \mathbb{C}$. Note that if we use the coordinates $y^{1}, \ldots, y^{n}$, we have the identity

$$
\exp (\theta)=\prod_{1}^{n}\left(1-\sqrt{-1} \frac{d y^{i} \wedge d \bar{y}^{i}}{2}\right) \text {. }
$$

Proposition 1.5. The operator $S \in \operatorname{End}\left(\Lambda\left(V_{R}\right) \otimes_{R} \mathbb{C}\right)$ is self-adjoint. Its lowest eigenvalues is equal to $-n$, and the corresponding eigenspace is spanned by the form of degree zero $\exp (\theta)$.

Proof. By (1.21), we know that

$$
S=\sqrt{-1} \sum_{1}^{n}\left(\frac{1}{2} d y^{i} \wedge d \bar{y}^{i}-2 i_{\partial / \partial \bar{y}^{i}} i_{\partial / \partial y^{i}}\right) .
$$

The operators in the sum in the right-hand side of (1.26) commute, so we may as well assume that $n=1$. One then easily verifies that $S$ has three distinct eigenvalues:

- the eigenvalue +1 , the corresponding eigenspace being spanned by $1+$ $\sqrt{-1} d y^{1} \wedge d \bar{y}^{1} / 2$

- the eigenvalue 0 , the corresponding eigenspace being spanned by $d y^{1}$ and $d \bar{y}^{1}$;

- the eigenvalue -1 , the corresponding eigenspace being spanned by $1-$ $\sqrt{-1} d y^{1} \wedge d \bar{y}^{1} / 2$.

The proposition is proved.

Let $\Gamma_{0}$ be the set of elements of $\Gamma$ which have compact support.

Theorem 1.6. The operator $\left(\bar{\partial}+\sqrt{-1} i_{y}+\bar{\partial}^{*}-\sqrt{-1} i_{y}^{*}\right)^{2}$ is essentially self-adjoint on $\Gamma_{0}$. Its closure has discrete spectrum and finite-dimensional eigenspaces. If $\beta \in \Gamma^{0}$ is given by

$$
\beta=\exp \left(\theta-|Y|^{2} / 2\right)
$$


then $\beta$ has total degree zero, and also

$$
\left(\frac{1}{2 \pi}\right)^{n} \int_{V_{R}} \beta \wedge * \beta=1 .
$$

$\beta$ spans the one-dimensional kernel of the operator $\left(\bar{\partial}+\sqrt{-1} i_{y}+\bar{\partial}^{*}-\sqrt{-1} i_{y}^{*}\right)^{2}$. Finally,

$$
\begin{gathered}
\left(\bar{\partial}+\sqrt{-1} i_{y}\right) \beta=0, \\
\left(\bar{\partial}^{*}-\sqrt{-1} i_{y}^{*}\right) \beta=0, \\
\left\langle\left(N_{H}-n / 2\right) \beta, \beta\right\rangle=\left\langle\left(N_{V}-n / 2\right) \beta, \beta\right\rangle=0 .
\end{gathered}
$$

Proof. Let $C_{0}^{\infty}$ be the set of $C^{\infty}$ functions on $V_{R}$ with values in $R$ which have compact support. Let $\mathscr{L}$ be the partial differential operator:

$$
\mathscr{L}=\frac{1}{2}\left(-\Delta+|Y|^{2}-2 n\right) \text {. }
$$

$\mathscr{L}$ is the harmonic oscillator on $V_{R}$. By [GIJ, Theorem 1.5.1], we know that $\mathscr{L}$ is essentially self-adjoint on $C_{0}^{\infty}$, that its closure is nonnegative, and that it has compact resolvent. In particular, the kernel of $\mathscr{L}$ is one-dimensional and spanned by the function $\exp \left(-|Y|^{2} / 2\right) . \mathscr{L}$ also acts naturally on $\Gamma$.

The matrix operator $S$ commutes with $\mathscr{L}$. Using Propositions 1.4 and 1.5 , we then find that the operator $\left(\bar{\partial}+\sqrt{-1} i_{y}+\bar{\partial}^{*}-\sqrt{-1} i_{y}^{*}\right)^{2}$ is essentially self-adjoint on $\Gamma_{0}$, and that $\beta$ spans its one-dimensional kernel.

Now by (1.14), we get

$$
\left|1-\sqrt{-1} d y^{i} \wedge d \bar{y}^{i} / 2\right|^{2}=2 .
$$

Also

$$
\int_{V_{R}} \exp -\left(|Y|^{2}\right) d v=\pi^{n}
$$

From (1.31) and (1.32), we get (1.28). Since $\theta$ has total degree zero, $\beta$ also has total degree zero. Clearly,

$$
|Y|^{2}=\sum_{1}^{n}\left|y^{i}\right|^{2}
$$

Using Proposition 1.2, (1.9), and (1.33), we get

$$
\left(\bar{\partial}+\sqrt{-1} i_{y}\right)\left(\theta-|Y|^{2} / 2\right)=0,
$$

and so

$$
\left(\bar{\partial}+\sqrt{-1} i_{y}\right) \exp \left(\theta-|Y|^{2} / 2\right)=0 .
$$

If $n=1$, still using Proposition 1.2, (1.9), and (1.33), we have

$$
\begin{aligned}
\left(\bar{\partial}^{*}-\right. & \left.\sqrt{-1} i_{y}^{*}\right) \exp \left(\theta-\frac{|Y|^{2}}{2}\right) \\
& =\left(\bar{\partial}^{*}-\sqrt{-1} i_{y}^{*}\right)\left(1-\frac{\sqrt{-1}}{2} d y^{1} \wedge d \bar{y}^{1}\right) \exp \left(\frac{-|Y|^{2}}{2}\right)=0 .
\end{aligned}
$$


The second line in (1.29) follows when $n=1$. By using the more general formula (1.25), we get the second line of (1.29) in full generality.

Observe that $N_{H}$ is a derivation on $\Lambda\left(V_{R}^{*}\right) \otimes_{R} \mathbb{C}$. Then since $N_{H} \theta=\theta$, we get

$$
N_{H} \beta=\left(N_{H} \theta\right) \beta=\theta \beta .
$$

Using (1.9) and (1.25), we find that

$$
\theta \beta=-\sum_{j=1}^{n} \sqrt{-1} \frac{d y^{j} \wedge d \bar{y}^{j}}{2} \prod_{k \neq j}\left(1-\sqrt{-1} \frac{d y^{k} \wedge d \bar{y}^{k}}{2}\right) \exp \left(\frac{-|Y|^{2}}{2}\right),
$$

and so

$$
\langle\theta \beta, \beta\rangle=\left(\frac{1}{2 \pi}\right)^{n} \sum_{j=1}^{n} 2^{n-1} \pi^{n}=\frac{n}{2} .
$$

From (1.37) and (1.39), we deduce that

$$
\left\langle\left(N_{H}-n / 2\right) \beta, \beta\right\rangle=0 \text {. }
$$

The proof that $\left\langle\left(N_{V}-n / 2\right) \beta, \beta\right\rangle=0$ is identical.

Remark 1.7. The vector field $\mathbf{J} Y=\sqrt{-1}(y-\bar{y})$ is a holomorphic Killing vector field on $V_{R}$. Also $\theta$ is $\mathbf{J} Y$ invariant, i.e.,

$$
L_{\mathrm{J} Y} \theta=0 \text {. }
$$

Let $\partial$ be the conjugate of the operator $\bar{\partial}$. The exterior differentiation operator $d$ is given by $d=\partial+\bar{\partial}$. Also

$$
\left(\bar{\partial}+\sqrt{-1} i_{y}\right)^{2}=0, \quad\left(\partial-\sqrt{-1} i_{\bar{y}}\right)^{2}=0 .
$$

We deduce from (1.41) that since $L_{\mathrm{J} Y}=\left(d+i_{\mathrm{J} Y}\right)^{2}$, then

$$
\left(\bar{\partial}+\sqrt{-1} i_{y}\right)\left(\partial-\sqrt{-1} i_{\bar{y}}\right) \theta+\left(\partial-\sqrt{-1} i_{\bar{y}}\right)\left(\bar{\partial}+\sqrt{-1} i_{y}\right) \theta=0 .
$$

We also easily see that

$$
\sqrt{-1}\left(\bar{\partial}+\sqrt{-1} i_{y}\right)\left(\partial-\sqrt{-1} i_{\bar{y}}\right) \theta=\theta-|Y|^{2} / 2 .
$$

Therefore,

$$
\beta=\exp \left\{\sqrt{-1}\left(\bar{\partial}+\sqrt{-1} i_{y}\right)\left(\partial-\sqrt{-1} i_{\bar{y}}\right) \theta\right\} .
$$

As observed in our earlier work [B3], the operators $\bar{\partial}+\sqrt{-1} i_{y}$ and $\partial-\sqrt{-1} i_{\bar{y}}$ are intimately related with the de Rham equivariant theory of $V_{R}$ with respect to the action of the holomorphic Killing vector field $\mathbf{J} Y$. Perhaps more surprising is that the form $\beta$ was also considered in an entirely different context in [B3] in relation with localization formulas in complex equivariant cohomology, along the lines of our version [B2, Theorem 1.3] of localization formulas in equivariant cohomology. 
(d) The cohomology of the complex $\left(\Gamma^{0}, \bar{\partial}+\sqrt{-1} i_{y}\right)$. Recall that due to $(1.23)$

$$
\begin{aligned}
& \left(\bar{\partial}+\sqrt{-1} i_{y}+\bar{\partial}^{*}-\sqrt{-1} i_{y}^{*}\right)^{2} \\
& \quad=\left(\bar{\partial}+\sqrt{-1} i_{y}\right)\left(\bar{\partial}^{*}-\sqrt{-1} i_{y}^{*}\right)+\left(\bar{\partial}^{*}-\sqrt{-1} i_{y}^{*}\right)\left(\bar{\partial}+\sqrt{-1} i_{y}\right) .
\end{aligned}
$$

Also if $f, g \in \Gamma_{0}$, then

$$
\begin{aligned}
& \left\langle\left(\bar{\partial}+\sqrt{-1} i_{y}+\bar{\partial}^{*}-\sqrt{-1} i_{y}^{*}\right)^{2} f, g\right\rangle \\
& \quad=\left\langle\left(\bar{\partial}+\sqrt{-1} i_{y}\right) f,\left(\bar{\partial}+\sqrt{-1} i_{y}\right) g\right\rangle+\left\langle\left(\bar{\partial}^{*}-\sqrt{-1} i_{y}^{*}\right) f,\left(\bar{\partial}^{*}-\sqrt{-1} i_{y}^{*}\right) g\right\rangle .
\end{aligned}
$$

Set

$$
\begin{aligned}
& Z=\left\{f \in \Gamma^{0},\left(\bar{\partial}+\sqrt{-1} i_{y}\right) f=0\right\}, \\
& B=\left\{f \in \Gamma^{0}, \exists g \in \Gamma^{0}, f=\left(\bar{\partial}+\sqrt{-1} i_{y}\right) g\right\}, \\
& H^{*}\left(\Gamma^{0}, \bar{\partial}+\sqrt{-1} i_{y}\right)=Z / B .
\end{aligned}
$$

Then one verifies easily as in [B4, $\S 2 \mathrm{~h})]$ that, using (1.23) and the properties of the harmonic oscillator, one can develop the Hodge theory for the $L_{2}$ cohomology of the complex $\left(\Gamma^{0}, \bar{\partial}+\sqrt{-1} i_{y}\right)$ which is formally similar to the usual Hodge theory for compact manifolds. In particular, there is a canonical isomorphism of $\mathbb{Z}$ graded vector spaces

$$
H^{*}\left(\Gamma^{0}, \bar{\partial}+\sqrt{-1} i_{y}\right) \cong \operatorname{Ker}\left(\bar{\partial}+\sqrt{-1} i_{y}+\bar{\partial}^{*}-\sqrt{-1} i_{y}^{*}\right)^{2} .
$$

From Theorem 1.6, we immediately deduce.

Theorem 1.8. For $p \neq 0, H^{p}\left(\Gamma^{0}, \bar{\partial}+\sqrt{-1} i_{y}\right)=\{0\}$. Also $H^{0}\left(\Gamma^{0}, \bar{\partial}+\sqrt{-1} i_{y}\right)$ is one-dimensional, and spanned by the $\bar{\partial}+\sqrt{-1} i{ }_{y} \quad L_{2}$ cohomology class of $\beta$.

Remark 1.9. Let $t \in R \rightarrow||_{t}$ be a smooth family of Hermitian metrics on $V$, and let $t \in R \rightarrow \theta_{t}$ be the corresponding family of Kähler forms. Set

$$
\beta_{t}=\exp \left(\theta_{t}-|Y|_{t}^{2} / 2\right) \text {. }
$$

Using (1.45), we find that

$$
\frac{\partial \beta_{t}}{\partial t}=\sqrt{-1}\left(\bar{\partial}+\sqrt{-1} i_{y}\right)\left(\partial-\sqrt{-1} i_{\bar{y}}\right)\left(\frac{\partial \theta_{t}}{\partial t} \beta_{t}\right) .
$$

(1.51) shows directly that the $L_{2}$ cohomology class of $\beta_{t}$ does not vary with $t$. In particular, if $\theta$ is taken as before, we get

$$
\begin{aligned}
& \frac{\partial}{\partial t} \exp \left\{\sqrt{-1}\left(\bar{\partial}+\sqrt{-1} i_{y}\right)\left(\partial-\sqrt{-1} i_{\bar{y}}\right) t \theta\right\} \\
& \quad=\sqrt{-1}\left(\bar{\partial}+\sqrt{-1} i_{y}\right)\left(\partial-\sqrt{-1} i_{\bar{y}}\right)\left(\theta \exp \left\{\sqrt{-1}\left(\bar{\partial}+\sqrt{-1} i_{y}\right)\left(\partial-\sqrt{-1} i_{\bar{y}}\right) t \theta\right\}\right) .
\end{aligned}
$$

By integrating (1.52) from 0 to 1 , we find in particular that $\beta$ is cohomologous in $\left(\Gamma, \bar{\partial}^{V}+\sqrt{-1} i_{y}\right)$ to the function 1 (which is not square-integrable!). If we use the notation of Proposition 1.1, this is related to the fact that

$$
r \beta=1 \text {. }
$$


We have thus shown that the complexes $\left(\Gamma, \bar{\partial}+\sqrt{-1} i_{y}\right)$ and $\left(\Gamma^{0}, \bar{\partial}+\sqrt{-1} i_{y}\right)$ have the same cohomology. Equivalently, the complex $\left(\Gamma^{0}, \bar{\partial}+\sqrt{-1} i_{y}\right)$ calculates the cohomology of the point 0 .

Also observe that if - denotes Fourier transform, then

$$
\begin{gathered}
\widehat{\bar{\partial}+\sqrt{-1} i_{y}}=\partial^{*}+\sqrt{-1} i_{\bar{y}}^{*}, \\
\overline{\bar{\partial}^{*}-\sqrt{-1} i_{y}^{*}}=\partial-\sqrt{-1} i_{\bar{y}}, \\
\hat{\beta}=(2 \pi)^{n} \beta .
\end{gathered}
$$

Of course the third line in (1.54) can be viewed as a consequence of the first two lines in (1.54).

Remark 1.10. Consider the Witten operator $[W]$

$$
d^{\prime}=e^{-|Y|^{2} / 2} d e^{|Y|^{2} / 2}
$$

and its formal adjoint $d^{\prime *}$, and form the Witten Laplacian $d^{\prime} d^{\prime *}+d^{\prime *} d^{\prime}$. The operator $d^{\prime}+d^{\prime *}$ is then given by a formula closely related to the second line of (1.23). The formula for the Witten Laplacian is very close to the fourth line of (1.23), and the study of the corresponding harmonic forms [W] is immediately related to the proof of Theorem 1.6.

Still these formal analogies obscure the fact that the analysis which was previously done makes use of the complex structure of $V_{R}$ in an essential way. This will become clearer in the sequel, where no real analogue of what is done can be found.

\section{THE HODGE THEORY ON THE FIBERS OF A HOLOMORPHIC VECTOR BUNDLE}

Let $B$ be a compact complex manifold and let $N$ be a holomorphic Hermitian vector bundle on $B$. In this section, we apply the results of $\S 1$ to the fibers of $N$. In particular, the form $\beta \in \Lambda\left(\bar{N}^{*}\right) \hat{\otimes} \Lambda\left(N^{*}\right)$ is now a canonical trivialization of the $L_{2}$ cohomology of the double complex $\left(\Gamma\left(\Lambda\left(\bar{N}^{*}\right) \hat{\otimes} \Lambda\left(N^{*}\right)\right), \bar{\partial}+\right.$ $\sqrt{-1} i_{y}$ ) in the fibers of $N_{R}$.

Also if

$$
0 \rightarrow L \underset{i}{\rightarrow} M \underset{j}{\rightarrow} N \rightarrow 0
$$

is an exact sequence of holomorphic vector bundles, where $M$ is equipped with a hermitian metric $g^{M}$ which induces Hermitian metrics $g^{L}$ and $g^{N}$ on $L$ and $N$, then we identify the holomorphic Hermitian connection $\nabla^{L}$ on $\Lambda\left(\bar{L}^{*}\right)$ with the natural connection on the $L^{2}$ cohomology in the fibers $N_{R}$ of the double complex $\left(\Gamma\left(\Lambda\left(\bar{M}^{*}\right) \hat{\otimes} \Lambda\left(N^{*}\right)\right), \bar{\partial}+\sqrt{-1} i_{y}\right)$.

This section is organized as follows. In (a) we apply the results of $\S 1$ to the vector bundle $N$. In (b), we calculate the natural connection on the $L_{2}$ cohomology of the complex $\left(\Gamma\left(\Lambda\left(\bar{M}^{*}\right) \hat{\otimes} \Lambda\left(N^{*}\right)\right), \bar{\partial}+\sqrt{-1} i_{y}\right)$.

(a) A double complex on a vector bundle. Let $B$ be a compact connected complex manifold of complex dimension $k$, let $N$ be a complex holomorphic vector 
bundle on $B$ of complex dimension $n$, let $\pi$ be the projection $N \rightarrow B$, and let $g^{N}$ be a smooth Hermitian metric on $N$. For $x \in B$, let $\theta_{x} \in \Lambda\left(N_{R}^{*}\right)$ be the Kähler form of the fiber $N_{x}$, which we define as in (1.7).

Let $\nabla^{N}$ be the holomorphic Hermitian connection on the vector bundle $N$. $\nabla^{N}$ induces a connection on $\Lambda\left(N_{R}^{*}\right)$, which we still denote $\nabla^{N}$.

For $x \in B, \Gamma_{x}$ denotes the set of smooth sections of $\Lambda\left(N_{R}^{*}\right)_{x} \otimes_{R} \mathbb{C}$ on the fiber $N_{R, x}$ and $\Gamma_{x}^{0}$ is the set of square-integrable sections of $\Lambda\left(N_{R}^{*}\right)_{x} \otimes_{R} \mathbb{C}$ on the fiber $N_{R, x}$. We equip $\Gamma_{x}^{0}$ with the Hermitian product

$$
f, g \in \Gamma_{x}^{0} \rightarrow\langle f, g\rangle=\left(\frac{1}{2 \pi}\right)^{n} \int_{N_{R, x}} f \wedge * g .
$$

As in [B1, §1f)], we will consider $\Gamma$ as an infinite-dimensional vector bundle on $B$. The set of smooth sections of $\Gamma$ is identified with the set of smooth sections of $\Lambda\left(N_{R}^{*}\right) \otimes_{R} \mathbb{C}$ on the total space of $N$.

Let $N_{V}$ and $N_{H}$ be the operators which define the $\mathbb{Z}$ grading of $\Lambda\left(\bar{N}^{*}\right)$ and $\Lambda\left(N^{*}\right)$. As in $\S 1(\mathrm{a})$, we grade $\Lambda\left(N_{R}^{*}\right) \otimes_{R} \mathbb{C}=\Lambda\left(\bar{N}^{*}\right) \widehat{\otimes} \Lambda\left(N^{*}\right)$ by the operator $N_{V}-N_{H}$. We then obtain a $\mathbb{Z}$ grading on $\Gamma$ and $\Gamma^{0}$.

We now denote by $\bar{\partial}^{N_{x}}$ the Dolbeault operator acting on the fiber $\Gamma_{x}$. If $y \in N_{x}$, the interior multiplication operator $i_{y}$ also acts on $\Gamma_{x}$.

The connection $\nabla^{N}$ defines a complex subbundle $T^{H} N$ of $T N$ such that we have the identification of $C^{\infty}$ complex vector bundles on $N$ :

$$
T N=N \oplus T^{H} N \text {. }
$$

If $U \in T_{R} B$, let ${ }^{0} U^{H}$ be the lift of $U$ in $T_{R}^{H} N$, so that ${ }^{0} U_{H} \in T_{R}^{H} N$ and $\pi_{*}^{0} U^{H}=U$.

Definition 2.1. If $h$ is a smooth section of $\Gamma$ on $B$ and $U \in T_{R} B$, set

$$
\widetilde{\nabla}_{U}^{\Gamma} h=\nabla_{0}^{N} U^{H} h .
$$

As in [B1, §1f)], one easily verifies that $\tilde{\nabla}^{\Gamma}$ is a connection on $\Gamma$.

Theorem 2.2. The curvature $\left(\tilde{\nabla}^{\Gamma}\right)^{2}$ of $\tilde{\nabla}^{\Gamma}$ is a $(1,1)$ form on $B$ with values in the set of formally skew-adjoint first order differential operators acting on the fibers $\Gamma$. Also

$$
\begin{gathered}
{\left[\tilde{\nabla}^{\Gamma}, \bar{\partial}^{N}+\sqrt{-1} i_{y}\right]=0 ; \quad\left[\tilde{\nabla}^{\Gamma}, \bar{\partial}^{N *}-\sqrt{-1} i_{y}^{*}\right]=0,} \\
{\left[\tilde{\nabla}^{\Gamma},\left(\bar{\partial}^{N}+\sqrt{-1} i_{y}+\bar{\partial}^{N *}-\sqrt{-1} i_{y}^{*}\right)^{2}\right]=0 .}
\end{gathered}
$$

If $\mu=\operatorname{Ker}\left(\bar{\partial}^{N}+\sqrt{-1} i_{y}+\bar{\partial}^{N *}-\sqrt{-1} i_{y}^{*}\right)^{2}, \mu$ is a one-dimensional trivial line bundle on $B$ which is spanned by

$$
\beta=\exp \left(\theta-|Y|^{2} / 2\right) ; \quad Y \in N_{R} .
$$


$\beta$ has total degree zero and for any $x \in B$

$$
\left(\frac{1}{2 \pi}\right)^{n} \int_{N_{R, x}} \beta_{x} \wedge * \beta_{x}=1
$$

Also

$$
\begin{gathered}
\tilde{\nabla}^{\Gamma} \beta=0, \\
\left(\bar{\partial}^{N}+\sqrt{-1} i_{y}\right) \beta=0, \\
\left(\bar{\partial}^{N *}-\sqrt{-1} i_{y}^{*}\right) \beta=0, \\
\left\langle\left(N_{H}-n / 2\right) \beta, \beta\right\rangle=\left\langle\left(N_{V}-n / 2\right) \beta, \beta\right\rangle=0 .
\end{gathered}
$$

Proof. Let $\left(\nabla^{N}\right)^{2}$ be the curvature of $\nabla^{N}$. By proceeding as in [B1, Proposition $1.11]$, one easily verifies that

$$
\left(\tilde{\nabla}^{\Gamma}\right)^{2}=\left(\nabla^{N}\right)^{2}-\nabla_{\left(\nabla^{N}\right)^{2} Y}
$$

$\left(\nabla^{N}\right)^{2}$ is a form on $B$ of type $(1,1)$ with values in matrix valued operators acting on $\Gamma .\left(\nabla^{N}\right)^{2} Y$ is a $(1,1)$ form on $B$ with values in $N_{R}$, and so $\nabla_{\left(\nabla^{N}\right)^{2} Y}$ is a $(1,1)$ form on $B$ with values in the set of first order differential operators acting on $\Gamma$. From (2.7), we find easily that $\left(\tilde{\nabla}^{\Gamma}\right)^{2}$ takes values in skew-adjoint first order differential operators acting on $\Gamma$.

The fact that $\mu$ is spanned by $\beta$ was proved in Theorem 1.6 , together with (2.5) and the last three lines of (2.6). (2.3) is trivial. Since $\nabla^{N}$ preserves the Hermitian metric $g^{N}$, then

$$
\widetilde{\nabla}^{\Gamma}\left(\theta-|Y|^{2} / 2\right)=0,
$$

and so $\tilde{\nabla}^{\Gamma} \beta=0$. Our theorem is proved.

Remark 2.3. By the first line in (2.6), it is clear that the connection $\tilde{\nabla}^{\Gamma}$ induces a Hermitian connection $\nabla^{\mu}$ on $\mu$, whose curvature vanishes. By [AHS, Theorem 5.1], there exists a holomorphic structure on $\mu$, and $\nabla^{\mu}$ is the corresponding holomorphic Hermitian connection on $\mu$. From (2.6), we find that $\beta$ is a holomorphic section of $\mu$ of norm 1, i.e., $\beta$ trivializes $\mu$ as a holomorphic Hermitian line bundle.

By imitating the argument in [BGS3, §3], where the holomorphic structure on the determinant of a direct image was constructed by the method of Grothendieck-Knudsen-Mumford [KM], one can prove that $\beta$ defines a holomorphic section of the trivial holomorphic line bundle $H^{0}\left(\Gamma^{0}, \bar{\partial}^{N}+\sqrt{-1} i_{y}\right)$.

(b) An enlarged double complex. Let

$$
0 \rightarrow L \underset{i}{\rightarrow} M \underset{j}{\rightarrow} N \rightarrow 0
$$

be a holomorphic acyclic chain complex of vector bundles $L, M$, and $N$ on the manifold $B$. Let $l, m$, and $n$ be the complex dimensions of $L, M$, and $N$. We identify $L$ with a holomorphic vector subbundle of $M$, and $N$ with $M / L$. 
Let $g^{M}$ be a smooth Hermitian metric on the vector bundle $M$. As a vector subbundle of $M, L$ inherits a smooth Hermitian metric $g^{L}$. We identify $N$ (as a smooth vector bundle on $B$ ) with the orthogonal $L^{\perp}$ to $L$ in $M$. Therefore $N$ is equipped with a Hermitian metric $g^{N}$. We then have the identity

$$
M=L \oplus N
$$

in the class of smooth Hermitian vector bundles on $Y$, but of course (2.9) is not in general a holomorphic splitting of $M$.

Let $P^{L}$ and $P^{N}$ be the orthogonal projection operators from $M$ on $L$ and $N$ respectively. Let $\nabla^{L}, \nabla^{M}$, and $\nabla^{N}$ be the holomorphic Hermitian connections on the vector bundles $L, M$, and $N$. We still denote by $\nabla^{L}$, $\nabla^{M}$, and $\nabla^{N}$ the connections induced by $\nabla^{L}, \nabla^{M}$, and $\nabla^{N}$ on the tensor algebras of $L, M$, and $N$.

If $f$ is a smooth section of $L$, it is clear that $\nabla^{L} f=P^{L} \nabla^{M} f$. We now prove the corresponding results for $\nabla^{N}$.

Proposition 2.4. If $f$ is a smooth section of the vector bundle $N$, then

$$
\nabla^{N} f=P^{N} \nabla^{M} f .
$$

Proof. Let $f$ be a locally defined holomorphic section of $N$. There is a locally defined holomorphic section $g$ of $M$ such that $j(g)=f$, and so if $h=g-f$, then $h \in L$. Clearly, $\nabla^{M^{\prime \prime}} g=0$, and so

$$
\nabla^{M^{\prime \prime}} h+\nabla^{M^{\prime \prime}} f=0
$$

Since $L$ is a holomorphic subbundle of $M, \nabla^{M^{\prime \prime}} h \in L$, and so from (2.12) we deduce that

$$
P^{N} \nabla^{M^{\prime \prime}} f=0 .
$$

From (2.13), we find that the connection $P^{N} \nabla^{M^{\prime \prime}}$ on $N$ is holomorphic. Since it preserves the metric of $N$, it coincides with $\nabla^{N}$.

Let ${ }^{0} \nabla^{M}=\nabla^{L} \oplus \nabla^{N}$ be the direct sum of the connections $\nabla^{L}$ and $\nabla^{N}$ on $M=L \oplus N$. Set

$$
A=\nabla^{M}-{ }^{0} \nabla^{M} .
$$

Then $A$ is a one-form on $B$ with values in skew-adjoint elements of $\operatorname{End}(M)$ which interchange $L$ and $N$.

From (2.10), we deduce the identification of smooth Hermitian vector bundles on $B$ :

$$
\Lambda\left(\bar{M}^{*}\right)=\Lambda\left(\bar{L}^{*}\right) \hat{\otimes} \Lambda\left(\bar{N}^{*}\right) .
$$

Of course the identification (2.15) depends on the metric $g^{M}$. It is not an identification of antiholomorphic vector bundles on $B$. From (2.15), we get the identification of smooth vector bundles:

$$
\Lambda\left(\bar{M}^{*}\right) \hat{\otimes} \Lambda\left(N^{*}\right)=\Lambda\left(\bar{L}^{*}\right) \hat{\otimes}\left(\Lambda\left(N_{R}^{*}\right) \otimes_{R} \mathbb{C}\right) .
$$


For $x \in B$, let $E_{x}$ (resp. $E_{x}^{0}$ ) be the set of smooth (resp. square-integrable) sections of $\left(\Lambda\left(\bar{M}^{*}\right) \hat{\otimes} \Lambda\left(N^{*}\right)\right)_{x}$ over the fiber $N_{R, x}$. As in $\S 2($ a), we consider $E$ and $E^{0}$ as infinite dimensional vector bundles on $B$. Let $d v_{N}$ be the volume element on $N_{R, x}$. We equip $E_{x}^{0}$ with the Hermitian product

$$
f, g \in E_{x}^{0} \rightarrow\langle f, g\rangle=\left(\frac{1}{2 \pi}\right)^{n} \int_{N_{R, x}}\langle f, g\rangle d v_{N} .
$$

Let $N^{L}$ be the operator defining the $\mathbb{Z}$ grading on $\Lambda\left(\bar{L}^{*}\right)$.

As in $\S 2(\mathrm{a}), N_{V}$ and $N_{H}$ denote the operators defining the $\mathbb{Z}$ grading on $\Lambda\left(\bar{N}^{*}\right)=\bigoplus_{p=0}^{n} \Lambda^{p}\left(\bar{N}^{*}\right)$ and $\Lambda\left(N^{*}\right)=\bigoplus_{p=0}^{n} \Lambda^{p}\left(N^{*}\right)$ respectively. The operator $N_{V}-N_{H}$ defines the $\mathbb{Z}$ grading on $\Lambda\left(N_{R}^{*}\right) \otimes_{R} \mathbb{C}=\Lambda\left(\bar{N}^{*}\right) \widehat{\otimes} \Lambda\left(N^{*}\right)$. The $\mathbb{Z}$ grading on $\Lambda\left(\bar{L}^{*}\right) \widehat{\otimes}\left(\Lambda\left(N_{R}^{*}\right) \otimes_{R} \mathbb{C}\right)$ is defined by the operator $N^{L}+N_{V}-N_{H}$.

Let $\nabla$ denote the connection on $\Lambda\left(\bar{M}^{*}\right) \widehat{\otimes} \Lambda\left(N^{*}\right)$ :

$$
\nabla=\nabla^{M} \otimes 1+1 \otimes \nabla^{N}
$$

$y$ denotes the general element in $N$ and $Y=y+\bar{y}$ is the corresponding element in $N_{R}$. For every $x \in B$, the operator $\bar{\partial}^{N_{x}}+\sqrt{-1} i_{y}$ and its formal adjoint $\bar{\partial}^{N_{x} *}-\sqrt{-1} i_{y}^{*}$ act on $E_{x}$.

If $U \in T_{R} B,{ }^{0} U^{H}$ still denotes the horizontal lift of $U$ in $T_{R}^{H} N .{ }^{0} U^{H}$ was explicitly constructed in $\S 2(a)$.

Definition 2.5. If $h$ is a smooth section of $E$ and $U \in T_{R} B$, set

$$
\tilde{\nabla}_{U}^{E} h=\nabla_{U_{U}} h .
$$

$\tilde{\nabla}^{E}$ is a connection on the infinite-dimensional vector bundle $E$.

Remark 2.6. By (2.16), we have an isomorphism of $\mathbb{Z}$ graded vector bundles

$$
\begin{aligned}
E & =\Lambda\left(\bar{L}^{*}\right) \widehat{\otimes} \Gamma, \\
E^{0} & =\Lambda\left(\bar{L}^{*}\right) \hat{\otimes} \Gamma^{0} .
\end{aligned}
$$

$E$ is a $\Lambda\left(\bar{L}^{*}\right)$ graded module, and the operators $\bar{\partial}^{N}+\sqrt{-1} i_{y}$, and $\bar{\partial}^{N *}-\sqrt{-1} i_{y}^{*}$ are $\Lambda\left(\bar{L}^{*}\right)$ linear.

It is essential to observe that since the identification $M=L \oplus N$ is only an identification of smooth vector bundles, in general the connection $\tilde{\nabla}^{E}$ on $E=\Lambda\left(\bar{L}^{*}\right) \hat{\otimes} \Gamma$ does not coincide with the connection $\nabla^{L} \otimes 1+1 \otimes \widetilde{\nabla}^{\Gamma}$.

By $\S 1(\mathrm{~b}), \Lambda\left(\bar{L}^{*}\right)$ is a $c\left(L_{R}\right)$ Clifford module, $\Lambda\left(\bar{M}^{*}\right)$ is a $c\left(M_{R}\right)$ Clifford module, and so $\Lambda\left(\bar{M}^{*}\right)$ is also a $c\left(L_{R}\right)$ Clifford module. The identification $\Lambda\left(\bar{M}^{*}\right)=\Lambda\left(\bar{L}^{*}\right) \hat{\otimes} \Lambda\left(\bar{N}^{*}\right)$ is an identification of $c\left(L_{R}\right)$ Clifford modules.

From (2.20), we deduce that $E$ and $E^{0}$ are $c\left(L_{R}\right)$ Clifford modules. 
Theorem 2.7. For $x \in B$, set

$$
\nu_{x}=\left\{h \in E_{x}^{0} ; h \in \operatorname{Ker}\left(\bar{\partial}^{N_{x}}+\sqrt{-1} i_{y}+\bar{\partial}^{N_{x}^{*}}-\sqrt{-1} i_{y}^{*}\right)^{2}\right\} .
$$

Then the $\nu_{x}$ 's are the fibers of a complex vector bundle $\nu$, which is a $c\left(L_{R}\right)$ $C l i f f o r d$ submodule of $E$. If we equip $\nu$ with the Hermitian metric induced by the Hermitian metric on $E^{0}$, then the linear map

$$
\psi: \alpha \in \Lambda\left(\bar{L}^{*}\right) \rightarrow \alpha \beta \in \nu
$$

is an isomorphism of Hermitian $\mathbb{Z}$ graded $c\left(L_{R}\right)$ Clifford modules.

Let $Q$ be the orthogonal projection from $E^{0}$ on $\nu$ and let $\nabla^{\nu}$ be the connection on $\nu$ given by

$$
\nabla^{\nu}=Q \widetilde{\nabla}^{E}
$$

Then

$$
\psi_{*}\left(\nabla^{L}\right)=\nabla^{\nu}
$$

Proof. The first part of our theorem is a trivial consequence of Theorem 2.2 and of Remark 2.6. Clearly, we have the identity of connections on $L$

$$
\nabla^{L}=P^{L} \nabla^{M} \text {. }
$$

Also, by Proposition 2.4,

$$
\nabla^{N}=Q \nabla^{M}
$$

The Kähler form $\theta$ on $N$ lies in $\Lambda\left(N_{R}^{*}\right) \otimes_{R} \mathbb{C}=\Lambda\left(\bar{N}^{*}\right) \widehat{\otimes} \Lambda\left(N^{*}\right)$. Remember that $\Lambda\left(\bar{N}^{*}\right) \subset \Lambda\left(\bar{M}^{*}\right)$. So $\theta$ will be regarded as an element of $\Lambda\left(\bar{M}^{*}\right) \widehat{\otimes} \Lambda\left(N^{*}\right)$. $\beta$ is then considered as a section of $\Lambda\left(\bar{M}^{*}\right) \widehat{\otimes} \Lambda\left(N^{*}\right)$ over $N_{R}$.

Let $\alpha$ be a smooth section on $B$ of $\Lambda\left(\bar{L}^{*}\right) \subset \Lambda\left(\bar{M}^{*}\right)$. Then if $U \in T_{R} B$

$$
\tilde{\nabla}_{U}^{E}(\alpha \beta)=\left(\widetilde{\nabla}_{U}^{E} \alpha\right) \beta+\alpha \tilde{\nabla}_{U}^{E} \beta
$$

By definition,

$$
\widetilde{\nabla}_{U}^{E} \alpha=\nabla_{{ }^{H}}^{M} \alpha \text {. }
$$

$\operatorname{End}(M)$ acts as a derivation on the algebra $\Lambda\left(\bar{M}^{*}\right)$. From (2.14), we find that

$$
\nabla_{U}^{M} \alpha=\nabla_{U}^{L} \alpha+A(U) \alpha .
$$

Since $A(U)$ interchanges $L$ and $N$, we get

$$
N_{V} A(U) \alpha=A(U) \alpha .
$$

Since $N_{H} A(U) \alpha=0$, we find that

$$
\left(N_{V}-N_{H}\right) A(U) \alpha=A(U) \alpha .
$$

By Theorem 2.2, $\left(N_{V}-N_{H}\right) \beta=0$. From (2.31) we deduce that

$$
\left(N_{V}-N_{H}\right)((A(U) \alpha) \beta)=(A(U) \alpha) \beta
$$


By Theorem 2.2, we know that $\nu \subset \operatorname{Ker}\left(N_{V}-N_{H}\right)$. From (2.32), we deduce that since $N_{V}-N_{H}$ is a self-adjoint operator

$$
Q(A(U) \alpha) \beta=0 .
$$

From (2.27)-(2.29) and (2.33) we get

$$
Q\left(\tilde{\nabla}_{U}^{E} \alpha\right) \beta=\left(\nabla_{U}^{L} \alpha\right) \beta .
$$

By Theorem 2.2, we know that $\widetilde{\nabla}_{U}^{\Gamma} \beta=0$. Using (2.14) again, we obtain

$$
\tilde{\nabla}_{U}^{E} \beta=A(U) \beta .
$$

Note that in (2.35), $\beta$ is considered as an element of $\Lambda\left(\bar{M}^{*}\right) \hat{\otimes} \Lambda\left(N^{*}\right)$ and that $A(U)$ acts only on $\Lambda\left(\bar{M}^{*}\right)$ and not on $\Lambda\left(N^{*}\right)$. From (2.4), we get

$$
A(U) \beta=(A(U) \theta) \beta \text {. }
$$

Since $A(U)$ interchanges $L$ and $N$, we find that

$$
N_{V} A(U) \theta=0 ; \quad N_{H} A(U) \theta=A(U) \theta,
$$

and so

$$
\left(N_{V}-N_{H}\right) A(U) \theta=-A(U) \theta .
$$

Since $\left(N_{V}-N_{H}\right) \beta=0$, using (2.36) and (2.38), we get

$$
\left(N_{V}-N_{H}\right) \alpha A(U) \beta=-\alpha A(U) \beta \text {. }
$$

From (2.39), we deduce as before that

$$
Q \alpha A(U) \beta=0 .
$$

From (2.35) and (2.40), we obtain

$$
Q \alpha \widetilde{\nabla}_{U}^{E} \beta=0 .
$$

From (2.27), (2.34), and (2.41), we get

$$
Q \widetilde{\nabla}_{U}^{E}(\alpha \beta)=\left(\nabla_{U}^{L} \alpha\right) \beta
$$

Our theorem is proved.

\section{The LeVI-Civita SUPERCONNECTION OF AN ACYCLIC CHAIN COMPLEX}

Let

$$
0 \rightarrow L \underset{i}{\rightarrow} M \underset{j}{\rightarrow} N \rightarrow 0
$$

be a holomorphic acyclic chain complex of vector bundles on a complex manifold $B$. In this section, we introduce operators and superconnections naturally associated with the embedding $L \rightarrow M$.

Namely for $u>0$, we consider the family of operators $\bar{\partial}+\sqrt{-u} i_{j(y)}$ acting on the smooth sections of $\Lambda\left(\bar{M}^{*}\right) \hat{\otimes} \Lambda\left(N^{*}\right)$ over the fibers of $M_{R}$. If $M$ is equipped with a metric $g^{M}$ which induces metrics $g^{L}$ and $g^{N}$ on $L$ and $N$, we construct the family of Hodge Dirac operators acting on the fibers of $M_{R}$, 
and the associated Levi-Civita superconnection $\mathscr{B}_{u}$ in the sense of Bismut [B1, $\S 3]$. We also calculate the curvature $\mathscr{B}_{u}^{2}$ of the superconnection $\mathscr{B}_{u}$, and we establish highly nontrivial Bianchi identities.

This section is organized as follows. In (a), we introduce a family of Dirac operators acting on the fibers of $M$. In (b), we construct the Levi-Civita superconnection, and in (c) a twisted version of the Levi-Civita superconnection. Finally in (d), we establish Bianchi identities for the Levi-Civita superconnection.

The Levi-Civita superconnection of a Euclidean vector bundle with connection is intimately related to Index Theory. In particular, we show in Remark 3.7 that the Levi-Civita superconnection of the tangent space $T B$ is exactly the operator introduced by Getzler $[\mathrm{Ge}]$ at the final stage of his proof of the Atiyah-Singer Index Theorem.

(a) A family of Dirac operators on $M$. We now make the same assumptions and we use the same notations as in $\S 2(\mathrm{~b})$.

Definition 3.1. For $x \in B$, let $I_{x}$ (resp. $I_{x}^{0}$ ) be the set of smooth (resp. squareintegrable) sections of $\left(\Lambda\left(\bar{M}^{*}\right) \hat{\otimes} \Lambda\left(N^{*}\right)\right)_{x}$ over the fiber $M_{R, x}$.

The $\mathbb{Z}$ grading on $I_{x}$ is still defined by the operator $N^{L}+N_{V}-N_{H}$. Let $d v_{M}$ be the volume form on the fibers of $M_{R}$. We equip $I_{x}^{0}$ with the Hermitian product

$$
f, g \in I_{x}^{0} \rightarrow\langle f, g\rangle=\left(\frac{1}{2 \pi}\right)^{m} \int_{M_{x}}\langle f, g\rangle d v_{M}
$$

Let $\bar{\partial}^{M_{x}}$ be the Dolbeault operator acting on $I_{x}$, and let $\bar{\partial}^{M_{x} *}$ be its formal adjoint with respect to the Hermitian product (3.1) on $I_{x}^{0}$. Set

$$
D_{x}^{M}=\bar{\partial}^{M_{x}}+\bar{\partial}^{M_{x} *} \text {. }
$$

The connection $\nabla^{M}$ defines a natural splitting of $T M$ into

$$
T M=M \oplus T^{H} M .
$$

If $U \in T_{R} B$, let $U^{H}$ be the horizontal lift of $U$ in $T_{R}^{H} M$. If $\pi$ is the projection $M \rightarrow B$, then $U^{H}$ is characterized by the relations

$$
U^{H} \in T_{R}^{H} M, \quad \pi_{*} U^{H}=U .
$$

Remember that $\nabla$ is the connection on $\Lambda\left(\bar{M}^{*}\right) \hat{\otimes} \Lambda\left(N^{*}\right)$ :

$$
\nabla=\nabla^{M} \otimes 1+1 \otimes \nabla^{N} \text {. }
$$

Definition 3.2. If $h$ is a smooth section of the vector bundle $I$ and $U \in T_{R} B$, set

$$
\tilde{\nabla}_{U}^{I} h=\nabla_{U^{H}} h .
$$

Then $\tilde{\nabla}^{I}$ is a connection on the vector bundle $I$. Note that if $h$ is a smooth section of $I$ with compact support, then $h \in I^{0}$. If $U \in T_{R} B$, then $\widetilde{\nabla}_{U} h \in I$ 
has compact support, and so $\tilde{\nabla}_{U} h \in I^{0}$. One then easily verifies that $\tilde{\nabla}$ is a unitary connection on $I^{0}$, in the sense that if $h$ and $h^{\prime}$ are smooth sections of $I$ with compact support, then

$$
U\left\langle h, h^{\prime}\right\rangle=\left\langle\widetilde{\nabla}_{U} h, h^{\prime}\right\rangle+\left\langle h, \widetilde{\nabla}_{U} h^{\prime}\right\rangle .
$$

Let $R^{L}, R^{M}$, and $R^{N}$ be the curvatures of the connections $\nabla^{L}, \nabla^{M}$, and $\nabla^{N}$. We still denote by $R^{M}$ and $R^{N}$ the curvatures of the connections $\nabla^{M}$, and $\nabla^{N}$ on $\Lambda\left(\bar{M}^{*}\right)$ and $\Lambda\left(N^{*}\right)$. If $R$ is the curvature of the connection $\nabla$, then

$$
R=R^{M} \otimes I_{\Lambda\left(N^{*}\right)}+I_{\Lambda\left(\bar{M}^{*}\right)} \otimes R^{N} .
$$

If $y \in M$, let $\bar{y} \in \bar{M}$ be the conjugate of $y . y \in M$ represents $Y=y+\bar{y} \in$ $M_{R}$.

Proposition 3.3. If $U, V \in T_{R} B$, then $\left(\widetilde{\nabla}^{I}\right)^{2}(U, V)$ is the first order differential operator on $I$ :

$$
\left(\tilde{\nabla}^{I}\right)^{2}(U, V)=R(U, V)-\nabla_{R^{M}(U, V) Y} \cdot
$$

In particular, the two-form $\left(\widetilde{\nabla}^{I}\right)^{2}$ is of complex type $(1,1)$. Also

$$
\left[\tilde{\nabla}^{I}, D^{M}\right]=0 ; \quad\left[\tilde{\nabla}^{I}, \bar{\partial}^{M}\right]=0 ; \quad\left[\tilde{\nabla}^{I}, \bar{\partial}^{M *}\right]=0 .
$$

Proof. (3.8) follows from [B1, Proposition 1.11]. Since $R$ and $R^{M}$ are forms of type $(1,1),\left(\tilde{\nabla}^{I}\right)^{2}$ is also of type $(1,1)$. The holonomy group of the connection $\nabla^{M}$ consists of linear complex isometries of $M_{R}$. We then find that $\left[\nabla^{I}, \bar{\partial}^{M}\right]=0$ and $\left[\nabla^{I}, \bar{\partial}^{M *}\right]=0$. Our proposition is proved.

Remark 3.4. If $L_{R^{M}(U, V) Y}$ denotes the Lie derivative with respect to the vector field $R^{M}(U, V) Y,(3.8)$ can be rewritten in the form

$$
\left(\widetilde{\nabla}^{I}\right)^{2}=-L_{R^{M} Y}+I_{\Lambda\left(\bar{M}^{*}\right)} \otimes R^{N} .
$$

(b) The Levi-Civita superconnection of $M$. If $Y \in M_{R}$, then $R^{M} Y$ is a twoform on the manifold $B$ with values in $M_{R}$.

Remember that if $X \in M_{R}$, then $c(X)$ denotes the corresponding element in the Clifford algebra $c\left(M_{R}\right)$, and, by $\S 1(\mathrm{~b}), \Lambda\left(\bar{M}^{*}\right)$ and also $\Lambda\left(\bar{M}^{*}\right) \widehat{\otimes} \Lambda\left(N^{*}\right)$ are $c\left(M_{R}\right)$ Clifford modules.

Let $f_{1}, \ldots, f_{2 k}$ be a base of $T_{R} B$, and let $f^{1}, \ldots, f^{2 k}$ be the corresponding dual base of $T_{R}^{*} B$. Let $c\left(R^{M} Y\right) \in\left(\Lambda\left(T_{R}^{*} B\right) \widehat{\otimes} c\left(M_{R}\right)\right)^{\text {odd }}$ be given by

$$
c\left(R^{M} Y\right)=\frac{1}{2} \sum f^{\alpha} f^{\beta} c\left(R^{M}\left(f_{\alpha}, f_{\beta}\right) Y\right) .
$$

The $\mathbb{Z}$ grading of $\Lambda\left(\bar{M}^{*}\right) \hat{\otimes} \Lambda\left(N^{*}\right)$ induces a corresponding $\mathbb{Z}_{2}$ grading. Then the vector bundles $I$ and $I^{0}$ split into $I=I_{+} \oplus I_{-}$and $I^{0}=I_{+}^{0} \oplus I_{-}^{0}$. Therefore, we may apply to these $\mathbb{Z}_{2}$ graded vector bundles the superconnection formalism of Quillen [Q1]. In particular, the algebra End $I$ is naturally $\mathbb{Z}_{2}$ graded, 
with the even (resp. odd) elements in End $I$ preserving (resp. exchanging) $I_{+}$ and $I_{-}$. The computations which follow will be done in the graded algebra $\Lambda\left(T_{R}^{*} B\right) \widehat{\otimes}$ End $I$.

Definition 3.5. Let $\mathscr{A}$ be the superconnection on the $\mathbb{Z}_{2}$ graded vector bundle $I=I_{+} \oplus I_{-}$:

$$
\mathscr{A}=\widetilde{\nabla}^{I}+D^{M}-\frac{c\left(R^{M} Y\right)}{2 \sqrt{2}} .
$$

Note that $\pi: M \rightarrow B$ is a fibration whose fibers are vector spaces. Also as pointed out in (3.3), the connection $\nabla^{M}$ defines a horizontal complex vector bundle $T^{H} M \cong \pi^{*} T B$ such that $T M=M \oplus T^{H} M$.

We claim that $\mathscr{A}$ is exactly the Levi-Civita superconnection of the fibration $\pi: M \rightarrow B$ defined in Bismut $[\mathrm{B} 1, \S 3 \mathrm{c})]$ associated with the given metric on $M$, the horizontal vector bundle $T^{H} M$ and the connection $\nabla^{N}$ on $\Lambda\left(N^{*}\right)$ (with the factor $t$ in [B1, §3c)] set equal to $\frac{1}{2}$ ). In fact the vector bundle with connection $M$ is a $U(m)$ vector bundle. The situation described here is then a special case of the $G$-equivariant situation considered in [B1, §3, Remark 2]. The fact that $\mathscr{A}$ is the Levi-Civita superconnection now follows easily from the considerations in [B1].

Since $T_{R}^{H} M \cong \pi^{*} T_{R} B$, we have an isomorphism of smooth vector bundles

$$
\Lambda\left(T_{R}^{*} M\right)=\Lambda\left(T_{R}^{*} B\right) \widehat{\otimes} \Lambda\left(M_{R}^{*}\right) .
$$

Therefore if $\alpha \in \Lambda\left(M_{R}^{*}\right)$, we may associate to $\alpha$ a form in $\Lambda\left(T_{R}^{*} M\right)$ which we still note $\alpha$. Let $\gamma$ be the Kähler form of the fibers $M_{R}$. One then easily verifies that

$$
\bar{\partial} \partial|y|^{2}=-2 i \gamma+\left\langle R^{M} y, \bar{y}\right\rangle .
$$

From (3.14), we deduce that the fibration $\pi: M \rightarrow B$ is locally Kähler (at least when $\pi$ is restricted to bounded subsets of $M$ ), in the sense of [BGS3, Definition 1.25]. Therefore the considerations in [BGS3] can also be used in this situation.

Let $\operatorname{Tr}\left[R^{M}\right]$ be the trace of $R^{M} \in \Lambda^{2}\left(T_{R}^{*} B\right) \otimes \operatorname{End} M . \operatorname{Tr}\left[R^{M}\right]$ is a section of $\Lambda^{2}\left(T_{R}^{*} B\right)$. In the sequel, and to avoid possible confusion, we denote by $\widehat{R^{N}}$ the action of $R^{N}$ on $\Lambda N^{*} . \widehat{R^{N}}$ acts like $1 \otimes \widehat{R^{N}}$ on $\Lambda\left(\bar{M}^{*}\right) \widehat{\otimes} \Lambda\left(N^{*}\right)$.

Let $e_{1}, \ldots, e_{2 m}$ be an orthonormal base of $M_{R}$.

Theorem 3.6. The curvature $\mathscr{A}^{2}$ of the superconnection $\mathscr{A}$ is given by

$$
\mathscr{A}^{2}=-\frac{1}{2} \sum_{1}^{2 m}\left(\nabla_{e_{i}}+\frac{1}{2}\left\langle R^{M} Y, e_{i}\right\rangle\right)^{2}+\frac{1}{2} \operatorname{Tr}\left[R^{M}\right]+\widehat{R^{N}} .
$$

Proof. (3.15) can of course be proved directly. We will obtain (3.15) as a special case of formulas in Bismut [B1, Theorem 3.6]. Let $(\operatorname{det} T M)^{1 / 2}$ be a locally defined holomorphic square root of the line bundle det $T M$. Set

$$
F=\Lambda\left(\bar{M}^{*}\right) \otimes(\operatorname{det} T M)^{-1 / 2} \text {. }
$$


Then by [H, Theorem 2.2], $F$ can be identified with the $\mathbb{Z}_{2}$ graded Hermitian vector bundle of $T_{R} M$ spinors.

As explained after (3.14), the Levi-Civita superconnection $\mathscr{A}$ is a special case of the superconnection considered in a complex geometric setting in BismutGillet-Soule [BGS2, §2]. As shown in [BGS2, proof of Theorem 2.2], the formula of [B1, Theorem 3.6] for $\mathscr{A}^{2}$ (with $t=\frac{1}{2}$ ) can be used in this situation. In particular, with the notation of [B1, 11$]$, using [B1, (1.30)], $T$ is purely horizontal, i.e., if $U \in M_{R}$, then $i_{U} T=0$. Also if $V, W \in T_{R} B$ and $V^{H}, W^{H}$ denote the horizontal lifts of $V, W$ in $T_{R}^{H} M$, then one gets

$$
T\left(V^{H}, W^{H}\right)=R^{M}(V, W) Y .
$$

By [B1, (1.28)], we find that if $X \in M_{R}$, then the two-form $\langle S(X) \cdot, \cdot\rangle$ is purely horizontal. More precisely if $V, W \in T_{R} B$, we get from (3.16) that

$$
2\left\langle S(X) V^{H}, W^{H}\right\rangle=\left\langle R^{M}(V, W) Y, X\right\rangle .
$$

To obtain (3.15), we now use [B1, Theorem 3.6] with $t$ equal to $\frac{1}{2}$.

Remark 3.7. Note that by [BGS2, (1.41)], if $D^{\prime M}$ is the standard Dirac operator in the fibers $M_{R}$, then $D^{\prime M}=\sqrt{2} D^{M}$.

Assume temporarily that $M_{R}$ is instead an ordinary smooth Euclidean spin vector bundle on $B$ equipped with a Euclidean connection, and that $D^{\prime M}$ is now the family of Dirac operators acting on $M_{R}$ spinors. Let $\widetilde{\nabla}^{\prime}$ denote the obvious analogue of $\tilde{\nabla}^{I}$. In this case, the Levi-Civita superconnection $\mathscr{A}^{\prime}$ with parameter $t=\frac{1}{2}$ is given by the formula

$$
\mathscr{A}^{\prime}=\tilde{\nabla}^{\prime}+\frac{D^{\prime M}}{\sqrt{2}}-\frac{c\left(R^{M} Y\right)}{2 \sqrt{2}} .
$$

Using again [B1, Theorem 3.6], we obtain the formula

$$
\mathscr{A}^{\prime 2}=-\frac{1}{2} \sum_{1}^{2 \mathrm{dim} M}\left(\nabla_{e_{i}}+\frac{1}{2}\left\langle R^{M} Y, e_{i}\right\rangle\right)^{2} .
$$

Formulas (3.15) and (3.19) are very interesting. In fact if $M_{R}=T_{R} B$, the operator in the right-hand side of (3.19) is exactly the operator introduced by Getzler [Ge] in his proof of the local index theorem. It is remarkable that the Getzler operator-which is the limit of rescaled squares of Dirac operators on the manifold $B$-is itself the curvature of the Levi-Civita superconnection $\mathscr{A}^{\prime}$ associated with $M=T B$. Formulas (3.15) and (3.19) will be interpreted in the context of index theory in Remark 4.11.

(c) The Levi-Civita superconnection of the double complex. Let $\mathbf{J}$ denote the complex structure of $L, M$, or $N$. If $y^{\prime} \in N$, the operators $i_{y^{\prime}}$ and $i_{y^{\prime}}^{*}$ are odd operators in $\operatorname{End}\left(\Lambda\left(N^{*}\right)\right)$, i.e., they interchange $\Lambda^{\text {even }}\left(N^{*}\right)$ and $\Lambda^{\text {odd }}\left(N^{*}\right)$. $y^{\prime} \in N$ represents $Y^{\prime}=y^{\prime}+\bar{y}^{\prime} \in N_{R}$. For $Y^{\prime} \in N_{R}$, set

$$
V\left(Y^{\prime}\right)=\sqrt{-1}\left(i_{y^{\prime}}-i_{y^{\prime}}^{*}\right) \text {. }
$$


Equivalently, with the notations of $\S 1(b)$, we have

$$
V\left(Y^{\prime}\right)=\frac{\sqrt{-1}}{\sqrt{2}} \hat{c}\left(\mathbf{J} Y^{\prime}\right)
$$

The operators $i_{y^{\prime}}, i_{y^{\prime}}^{*}$, and $V\left(Y^{\prime}\right)$ also act naturally as odd operators on

$$
\Lambda\left(\bar{M}^{*}\right) \hat{\otimes} \Lambda\left(N^{*}\right)=\Lambda\left(\bar{L}^{*}\right) \widehat{\otimes}\left(\Lambda\left(N_{R}^{*}\right) \otimes_{R} \mathbb{C}\right) .
$$

Definition 3.8. For $u \geq 0, \mathscr{B}_{u}$ denotes the superconnection on the $\mathbb{Z}_{2}$ graded vector bundle $I=I_{+} \oplus I_{-}$:

$$
\mathscr{B}_{u}=\tilde{\nabla}^{I}+D^{M}+\sqrt{u} V\left(P^{N} Y\right)-\frac{c\left(R^{M} Y\right)}{2 \sqrt{2}} .
$$

Clearly,

$$
D^{M}+\sqrt{u} V\left(P^{N} Y\right)=\bar{\partial}^{M}+\sqrt{-u} i_{P^{N} y}+\bar{\partial}^{M *}-\sqrt{-u} i_{P^{N} y}^{*}
$$

Also

$$
\left(\bar{\partial}^{M}+\sqrt{-u} i_{P^{N} y}\right)^{2}=0 ; \quad\left(\bar{\partial}^{M *}-\sqrt{-u} i_{P^{N} y}^{*}\right)^{2}=0 .
$$

The connection $\tilde{\nabla}^{I}$ splits into

$$
\tilde{\nabla}^{I}=\widetilde{\nabla}^{I^{\prime}}+\widetilde{\nabla}^{I^{\prime \prime}}
$$

where $\tilde{\nabla}^{I^{\prime}}$ and $\widetilde{\nabla}^{I^{\prime \prime}}$ are the holomorphic and antiholomorphic parts of the connection $\tilde{\nabla}^{I}$. Set

$$
\begin{aligned}
& \mathscr{B}_{u}^{\prime}=\tilde{\nabla}^{I^{\prime}}+\bar{\partial}^{M *}+\sqrt{u} \frac{\hat{c}\left(P^{N} \bar{y}\right)}{\sqrt{2}}-\frac{c\left(R^{M} \bar{y}\right)}{2 \sqrt{2}}, \\
& \mathscr{B}_{u}^{\prime \prime}=\tilde{\nabla}^{I^{\prime \prime}}+\bar{\partial}^{M}-\sqrt{u} \frac{\hat{c}\left(P^{N} y\right)}{\sqrt{2}}-\frac{c\left(R^{M} y\right)}{2 \sqrt{2}} .
\end{aligned}
$$

Equivalently, using (1.15) and (1.17), we have

$$
\begin{aligned}
& \mathscr{B}_{u}^{\prime}=\widetilde{\nabla}^{I^{\prime}}+\bar{\partial}^{M *}-\sqrt{-u} i_{P^{N} y}^{*}+i_{R^{M} \bar{y} / 2}, \\
& \mathscr{B}_{u}^{\prime \prime}=\widetilde{\nabla}^{I^{\prime \prime}}+\bar{\partial}^{M}+\sqrt{-u} i_{P^{N} y}-i_{R^{M} \bar{y} / 2}^{*} .
\end{aligned}
$$

Proposition 3.9. The following identities hold:

$$
\begin{aligned}
& \mathscr{B}_{u}=\mathscr{B}_{u}{ }^{\prime}+\mathscr{B}_{u}{ }^{\prime \prime}, \\
& \mathscr{B}_{u}{ }^{2}=0 ; \quad \mathscr{B}_{u}{ }^{\prime 2}=0, \\
& \mathscr{B}_{u}{ }^{2}=\left[\mathscr{B}_{u}^{\prime}, \mathscr{B}_{u}^{\prime \prime}\right] .
\end{aligned}
$$

Proof. In view of the considerations in $\S 3$ (b), (3.27) follows from [BGS2, Theorem 2.6]. More directly, observe that by Proposition 3.3

$$
\left(\widetilde{\nabla}^{I^{\prime \prime}}\right)^{2}=0 ; \quad\left(\nabla^{I^{\prime}}\right)^{2}=0
$$


Also, by Proposition 3.3

$$
\left[\tilde{\nabla}^{I}, \bar{\partial}^{M}\right]=0 ; \quad\left[\tilde{\nabla}^{I}, \bar{\partial}^{M *}\right]=0 .
$$

Moreover the map $y \in M \rightarrow P^{N} y=j(y) \in N$ is holomorphic and so

$$
\left[\widetilde{\nabla}^{I^{\prime \prime}}, i_{P^{N}}\right]=0 \text {. }
$$

Taking adjoints in (3.30), we also get

$$
\left[\widetilde{\nabla}^{I^{\prime}}, i_{P^{N}}^{*}\right]=0 .
$$

By Bianchi's identities, we find that

$$
\left[\widetilde{\nabla}^{I}, i_{R^{M} \bar{y}}\right]=0, \quad\left[\widetilde{\nabla}^{I}, i_{R^{M} \bar{y}}^{*}\right]=0 .
$$

Finally since $i_{R^{M} \bar{y}}^{*}$ (resp. $i_{R^{M} \bar{y}}$ ) is a holomorphic (resp. antiholomorphic) function of $y$, we get

$$
\begin{aligned}
& \left(\bar{\partial}^{M}+\sqrt{-u} i_{P^{N} y}-i_{R^{M} \bar{y} / 2}^{*}\right)^{2}=0, \\
& \left(\bar{\partial}^{M *}-\sqrt{-u} i_{P^{N} y}^{*}+i_{R^{M} \bar{y} / 2}\right)^{2}=0 .
\end{aligned}
$$

(3.27) now follows from (3.28)-(3.33).

Remember that $A \in \Lambda^{1}\left(T_{R}^{*} B\right) \widehat{\otimes}$ End $M$ was defined in (2.14). In particular, for $U \in T_{R} B, A(U)$ exchanges $L$ and $N$. Let $f_{1}, \ldots, f_{2 k}$ be a real base of $T_{R} B$, and let $f^{1}, \ldots, f^{2 k}$ be the corresponding dual base of $T_{R}^{*} B$. Set

$$
\hat{c}\left(\mathbf{J} A P^{L} Y\right)=-\sum_{1}^{2 k} f^{j} \hat{c}\left(\mathbf{J} A\left(f_{j}\right) P^{L} Y\right) \in\left(\Lambda\left(T_{R}^{*} B\right) \widehat{\otimes} \operatorname{End} \Lambda\left(N^{*}\right)\right)^{\text {even }} .
$$

Our choice of signs in (3.34) takes into account the fact that $\hat{c}$ should be thought of as an odd operator.

Let $e_{1}, \ldots, e_{2 m}$ be an orthonormal base of $M_{R}$ as in Theorem 3.6. The operator $S \in$ End $^{\text {even }}\left(\Lambda\left(N^{*}\right) \widehat{\otimes} \Lambda\left(N^{*}\right)\right)$ can be defined as in (1.22). It obviously extends into an even operator acting on

$$
\Lambda\left(\bar{M}^{*}\right) \widehat{\otimes} \Lambda\left(N^{*}\right)=\Lambda\left(L^{*}\right) \widehat{\otimes}\left(\Lambda\left(\bar{N}^{*}\right) \widehat{\otimes} \Lambda\left(N^{*}\right)\right) .
$$

From (1.23), we deduce in particular that

$$
S=\frac{\sqrt{-1}}{2} \sum_{1}^{2 m} c\left(P^{N} e_{j}\right) \hat{c}\left(\mathbf{J} P^{N} e_{j}\right)
$$

Theorem 3.10. For any $u \geq 0$, the following identity holds:

$$
\begin{aligned}
\mathscr{B}_{u}^{2}= & -\frac{1}{2} \sum_{1}^{2 m}\left(\nabla_{e_{i}}+\frac{1}{2}\left\langle R^{M} Y, e_{i}\right\rangle\right)^{2}+\frac{u\left|P^{N} Y\right|^{2}}{2} \\
& +\sqrt{u} S+\frac{\sqrt{-1} \sqrt{u}}{\sqrt{2}} \hat{c}\left(\mathbf{J} A P^{L} Y\right)+\frac{1}{2} \operatorname{Tr}\left[R^{M}\right]+\widehat{R^{N}} .
\end{aligned}
$$


Proof. If $m$ is a section of $M_{R}$ such that $\nabla^{M} m=0$, then

$$
\nabla^{N} P^{N} m=-A P^{L} m \text {. }
$$

Formula (3.36) now follows from Proposition 1.4, Theorem 3.6, and (3.37).

(d) Bianchi's identities for the superconnection $\mathscr{B}_{u}$. The split connection ${ }^{0} \nabla^{M}$ $=\nabla^{L} \oplus \nabla^{N}$ was defined in $\S 2(\mathrm{~b}) .{ }^{0} \nabla^{M}$ induces a connection on $\Lambda\left(\bar{M}^{*}\right)=$ $\Lambda\left(\bar{L}^{*}\right) \hat{\otimes} \Lambda\left(\bar{N}^{*}\right)$. We still denote by ${ }^{0} \nabla^{M}$ the connection ${ }^{0} \nabla^{M} \otimes 1+1 \otimes \nabla^{N}$ on $\Lambda\left(\bar{M}^{*}\right) \hat{\otimes} \Lambda\left(N^{*}\right)$.

The connection ${ }^{0} \nabla^{M}$ defines a complex horizontal subbundle ${ }^{0} T^{H} M$ of $T M$. If $U \in T_{R} B$, let ${ }^{0} U^{H}$ be the lift of $U$ in ${ }^{0} T_{R}^{H} M$.

Definition 3.11. If $h$ is a smooth section of $I$ and $U \in T_{R} B$, set

$$
{ }^{0} \tilde{\nabla}_{U}^{I} h={ }^{0} \nabla_{0}^{M} U^{H} \text {. }
$$

${ }^{0} \widetilde{\nabla}^{I}$ is a connection on $I$.

If $U \in T_{R} B$, then $L_{A(U) Y}$ is the Lie derivative with respect to the vector field $A(U) Y . L_{A(U) Y}$ acts on smooth sections of $\Lambda\left(\bar{M}^{*}\right)$ and also as a standard derivation on smooth sections of $\Lambda\left(N^{*}\right)$. So $L_{A(U) Y}$ acts naturally on $I$.

Let $\bar{\partial}^{L}$ and $\bar{\partial}^{N}$ be the $\bar{\partial}$ operators on $L_{R}$ and $N_{R}$, and let $\bar{\partial}^{L *}$ and $\bar{\partial}^{N *}$ be their formal adjoints. These operators act on $I$ in the obvious way. In particular,

$$
\bar{\partial}^{M}=\bar{\partial}^{L}+\bar{\partial}^{N}, \quad \bar{\partial}^{M *}=\bar{\partial}^{L *}+\bar{\partial}^{N *} .
$$

Set

$$
D^{L}=\bar{\partial}^{L}+\bar{\partial}^{L *}, \quad D^{N}=\bar{\partial}^{N}+\bar{\partial}^{N *}
$$

Then

$$
D^{M}=D^{L}+D^{N}
$$

We now prove remarkable commutation relations verified by the curvature $\mathscr{B}_{u}^{2}$ of $\mathscr{B}_{u}$.

Theorem 3.12. The following identities hold:

Proof. By (2.14), if $U \in T_{R} B$, then

$$
\begin{gathered}
\tilde{\nabla}^{I}={ }^{0} \tilde{\nabla}^{I}-L_{A Y} \\
{\left[{ }^{0} \tilde{\nabla}^{I}-\nabla_{A Y}+D^{N}+\sqrt{u} V\left(P^{N} Y\right)-\frac{c\left(P^{N} R^{M} Y\right)}{2 \sqrt{2}}, \mathscr{B}_{u}^{2}\right]=0} \\
{\left[A+D^{L}-\frac{c\left(P^{L} R^{M} Y\right)}{2 \sqrt{2}}, \mathscr{B}_{u}^{2}\right]=0 .}
\end{gathered}
$$

$$
\nabla_{U}^{M}={ }^{0} \nabla_{U}^{M}+A(U) .
$$


From (3.43) it is clear that if $U \in T_{R} B$, then

$$
U^{H}={ }^{0} U^{H}-A(U) Y .
$$

From (3.43) and (3.44), we deduce that

$$
\tilde{\nabla}^{I}={ }^{0} \tilde{\nabla}^{I}-L_{A Y} .
$$

As pointed out after (3.38), $L_{A Y}$ acts like a Lie derivative on the algebra $\Lambda\left(\bar{M}^{*}\right)$ and like a standard derivation on $\Lambda\left(N^{*}\right)$. In particular $A$ acts like $A \otimes 1$ on $\Lambda\left(\bar{M}^{*}\right) \hat{\otimes} \Lambda\left(N^{*}\right)$. Also, we have the trivial

$$
\left[\mathscr{B}_{u}, \mathscr{B}_{u}^{2}\right]=0 .
$$

Using (3.22), (3.40), and (3.45), we rewrite (3.46) in the form

$$
\begin{aligned}
& {\left[{ }^{0} \widetilde{\nabla}^{I}-\nabla_{A Y}+D^{N}+\sqrt{u} V\left(P^{N} Y\right)-\frac{c\left(P^{N} R^{M} Y\right)}{2 \sqrt{2}}, \mathscr{B}_{u}^{2}\right]} \\
& \quad+\left[A+D^{L}-\frac{c\left(P^{L} R^{M} Y\right)}{2 \sqrt{2}}, \mathscr{B}_{u}^{2}\right]=0 .
\end{aligned}
$$

Remember that $\Lambda\left(\bar{M}^{*}\right) \hat{\otimes} \Lambda\left(N^{*}\right) \cong \Lambda\left(\bar{L}^{*}\right) \hat{\otimes} \Lambda\left(\bar{N}^{*}\right) \hat{\otimes} \Lambda\left(N^{*}\right)$. Formula (3.36) shows that $\mathscr{B}_{u}^{2}$ preserves the partial grading in $\Lambda\left(\bar{L}^{*}\right)$, or equivalently that

$$
\left[\mathscr{B}_{u}^{2}, N^{L}\right]=0 \text {. }
$$

On the other hand the operator

$$
{ }^{0} \tilde{\nabla}^{I}-\nabla_{A Y}+D^{N}+\sqrt{u} V\left(P^{N} Y\right)-\frac{c\left(P^{N} R^{M} Y\right)}{2 \sqrt{2}}
$$

also preserves the partial degree in $\Lambda\left(\bar{L}^{*}\right)$ while the operator

$$
A+D^{L}-\frac{c\left(P^{L} R^{M} Y\right)}{2 \sqrt{2}}
$$

does not preserve this partial degree. It is in fact the sum of two pieces, one increasing the partial degree by one, and the other decreasing it by one. It immediately follows that each of the two terms in the left-hand side of (3.47) vanishes identically. Our theorem is proved.

Remark 3.13. The third identity in (3.42) can be proved directly. In fact, an easy computation shows that

$$
\begin{gathered}
{\left[D^{L}-\frac{c\left(P^{L} R^{M} Y\right)}{2 \sqrt{2}}, \sum_{1}^{2 m}\left(\nabla_{e_{i}}+\frac{1}{2}\left\langle R^{M} Y, e_{i}\right\rangle\right)^{2}\right]=0,} \\
{[A, S]+\left[D^{L}, \frac{\sqrt{-1}}{\sqrt{2}} \hat{c}\left(\mathbf{J} A P^{L} Y\right)\right]=0,}
\end{gathered}
$$

from which the third line in (3.42) trivially follows. The second identity in (3.42) can also be proved along the same lines. 
Definition 3.14. For $x \in B$, let $\mathscr{J}_{x}$ (resp. $\mathscr{J}_{x}^{0}$ ) be the set of smooth (resp. square-integrable) sections of $\Lambda\left(N_{R}^{*}\right)_{x}=\left(\Lambda\left(\bar{N}^{*}\right) \hat{\otimes} \Lambda\left(N^{*}\right)\right)_{x}$ over the fiber $M_{R, x}$.

Clearly, $\mathscr{J}$ and $\mathscr{J}^{0}$ are $\mathbb{Z}_{2}$ graded vector bundles on $B$, and moreover

$$
I=\Lambda\left(\bar{L}^{*}\right) \hat{\otimes} \mathcal{J}, \quad I^{0}=\Lambda\left(\bar{L}^{*}\right) \hat{\otimes} \mathcal{J}^{0} .
$$

Definition 3.15. If $h$ is a smooth section of $\mathscr{J}$ and $U \in T_{R} B$, set

$$
{ }^{0} \widetilde{\nabla}_{U}^{\mathcal{J}} h=\nabla_{0}^{N} U^{H} h \text {. }
$$

Clearly, ${ }^{0} \tilde{\nabla}^{\mathscr{J}}$ is a connection on $\mathscr{J}$. Also by (3.53), $\mathscr{J}$ is embedded naturally in $I$ by the map $f \in \mathscr{J} \rightarrow 1 \otimes f \in I .{ }^{0} \widetilde{\nabla}^{I}$ preserves $\mathscr{J}$ and its restriction to $\mathscr{J}$ coincides with ${ }^{0} \tilde{\nabla}^{\mathscr{J}}$.

Moreover, as is obvious from formula (3.36), $\mathscr{B}_{u}^{2}$ can be considered as an even section of $\Lambda\left(T_{R}^{*} B\right) \widehat{\otimes}$ End $\mathscr{J}$, which acts like $1 \widehat{\otimes} \mathscr{B}_{u}^{2}$ on $I=\Lambda\left(\bar{L}^{*}\right) \widehat{\otimes} \mathscr{J}$.

Theorem 3.16. The following identity holds:

$$
\left[{ }^{0} \widetilde{\nabla}^{\mathscr{J}}-\nabla_{A Y}+D^{N}+\sqrt{u} V\left(P^{N} Y\right)-\frac{c\left(P^{N} R^{M} Y\right)}{2 \sqrt{2}}, \mathscr{B}_{u}^{2}\right]=0 .
$$

Proof. Taking into account the considerations after (3.53), (3.54) is an obvious consequence of Theorem 3.12.

\section{THE GENERALIZED SUPERTRACES OF THE SUPERCONNECTION HEAT KERNELS}

In this section, we construct the kernels $P_{s}^{x, u, b}\left(Y, Y^{\prime}\right)$ on the fibers $M_{R, x}$ associated with the operators $\exp \left(-s \mathscr{B}_{u}^{2}+s b N_{H}\right)$. We prove that the forms $\operatorname{Tr}_{s}\left[P_{s}^{x, u, b}(Y, Y)\right]$ on $B$ are invariant by translation by elements of $L_{R}$. By integration on $N_{R}$, we so define generalized supertraces $\operatorname{Tr}_{s}\left[\exp \left(-\mathscr{B}_{u}^{2}+b N_{H}\right)\right]$ which are smooth forms on $B$.

We prove the key results that the forms $\operatorname{Tr}_{s}\left[\exp \left(-\mathscr{B}_{u}^{2}\right)\right]$ are closed, and that their cohomology class does not depend on $u>0$. More precisely, we obtain the double transgression formulas $(0.4)$, which are the obvious analogue of [BGS1, Theorem 1.15; BGS2, Theorem 2.9] for generalized supertraces. We also prove that if $R^{M}$ and $R^{N}$ are the curvatures of the holomorphic Hermitian connections on $M$ and $N$, then as $u \rightarrow 0$

$$
\operatorname{Tr}_{s}\left[\exp \left(-\mathscr{B}_{u}^{2}\right)\right]=\frac{\operatorname{Tr}\left(-R^{M}\right)}{\operatorname{Tr}\left(-R^{N}\right)}+O(u) .
$$

The proof of this result involves local index cancellations techniques. It will be reestablished in $\S 7$ by the explicit computation of the forms $\operatorname{Tr}_{s}\left[\exp \left(-\mathscr{B}_{u}^{2}\right)\right]$.

This section is organized as follows. In (a), we construct the heat kernels $P_{s}^{x, u, b}\left(Y, Y^{\prime}\right)$. In (b), we prove that these kernels decay fast enough at infinity 
on $N_{R}$. In (c), we prove the translation invariance of $\operatorname{Tr}_{s}\left[P_{s}^{x, u, b}(Y, Y)\right]$. In (d), we construct the generalized supertraces $\operatorname{Tr}_{s}\left[\exp \left(-\mathscr{B}_{u}^{2}+b N_{H}\right)\right]$, and in (e), we establish the double transgression formulas (0.4). In (f), we study the asymptotics of these forms on $B$ as $u \rightarrow 0$. Finally in (g), we construct a modified version $\mathscr{C}_{u}$ of the superconnection $\mathscr{B}_{u}$.

Our assumptions and notations are the same as in $\S 3$.

(a) The heat kernel associated with $\exp \left(-s \mathscr{B}_{u}^{2}+s b N_{H}\right)$. From now on, $\mathscr{B}_{u}^{2}$ will be considered as a section of $\left(\Lambda\left(T_{R}^{*} B\right) \hat{\otimes} \text { End } \mathscr{J}\right)^{\text {even }}$. Also $N_{H}$ lies in $(\text { End } \mathscr{J})^{\text {even }}$. Therefore for $b \in \mathbb{C},-\mathscr{B}_{u}{ }^{2}+b N_{H}$ is a section of the bundle of algebras $\left(\Lambda\left(T_{R}^{*} B\right) \widehat{\otimes} \text { End } \mathscr{J}\right)^{\text {even }}$. Recall that $d v_{M}$ is the volume element in the fibers $M_{R}$.

We now claim that there is an unambiguously defined heat equation semigroup $\exp \left(-s \mathscr{B}_{u}^{2}+s b N_{H}\right) \quad(s \geq 0)$. More precisely there is a uniquely defined smooth kernel $P_{s}^{x, u, b}\left(Y, Y^{\prime}\right) \quad\left(s>0, x \in B, Y, Y^{\prime} \in M_{R, x}\right)$ such that if $h$ is a smooth bounded section of $\left(\Lambda\left(T_{R}^{*} B\right) \widehat{\otimes} \Lambda\left(N_{R}^{*}\right)\right)_{x}$, then

$$
\exp \left(-s \mathscr{B}_{u}^{2}+s b N_{H}\right) h(Y)=\int_{M_{R, x}} P_{s}^{x, u, b}\left(Y, Y^{\prime}\right) h\left(Y^{\prime}\right) \frac{d v_{M}\left(Y^{\prime}\right)}{(2 \pi)^{m}} .
$$

In fact, if $B$ is reduced to one point and if $\Delta^{M_{R}}$ is the standard Laplacian on $M_{R}$, then by (3.36)

$$
\mathscr{B}_{u}^{2}=-\frac{\Delta^{M_{R}}}{2}+\frac{u\left|P^{N} Y\right|^{2}}{2}+\sqrt{u} S .
$$

Since $\Delta^{M_{R}}=\Delta^{L_{R}}+\Delta^{N_{R}}$, we find that, if $B$ is reduced to a point, then

$$
\mathscr{B}_{u}^{2}=-\frac{\Delta^{L_{R}}}{2}+\left(-\frac{\Delta^{N_{R}}}{2}+\frac{u\left|P^{N} Y\right|^{2}}{2}+\sqrt{u} S\right) .
$$

Therefore $\mathscr{B}_{u}^{2}$ is the sum of half the standard Laplacian on $L_{R}$ and of the harmonic oscillator introduced in Proposition 1.4 on $N_{R}$. The existence and uniqueness of the heat kernel $P_{s}^{x, u, b}$ is now standard.

More generally, since the terms $\left\langle R^{M} Y, e_{i}\right\rangle$ and $\hat{c}\left(A P^{L} Y\right)$ contain Grassmann variables in $\Lambda\left(T_{R}^{*} B\right)$, Duhamel's formula can be used to construct the heat kernel $P_{s}^{u, x, b}\left(Y, Y^{\prime}\right)$ and prove its main properties. Also, we can use stochastic differential equations techniques to construct the semigroup $\exp \left(-s \mathscr{B}_{u}^{2}+s b N_{H}\right)$ and the kernel $P_{s}^{x, u, b}\left(Y, Y^{\prime}\right)$. This last method will be used in the following subsection to obtain rough estimates on the kernels $P_{s}^{x, u, b}\left(Y, Y^{\prime}\right)$.

(b) The decay at infinity on $N_{R}$ of $P_{s}^{x, u, b}\left(Y, Y^{\prime}\right)$. As we shall see in a precise form in subsection (c), the kernel $P_{s}^{x, u, b}\left(Y, Y^{\prime}\right)$ does not tend to 0 as $|Y| \rightarrow$ $+\infty$, or $\left|Y^{\prime}\right| \rightarrow+\infty$. 
Here we will show that if we restrict $Y$ and $Y^{\prime}$ to vary in $N_{R, x}$, then $P_{s}^{u, x, b}\left(Y, Y^{\prime}\right)$ tends to 0 as $|Y| \rightarrow+\infty$, or $\left|Y^{\prime}\right| \rightarrow+\infty$. More precisely, we have the following result.

Theorem 4.1. Let $a, a^{\prime}, c \in R$ such that $0<a<a^{\prime}<+\infty$ and $0<c<1$, and let $k \in \mathbb{N}$. There exist $C, C^{\prime}>0$ such that for any $x \in B, u \in\left[a, a^{\prime}\right]$, $s \in[c, 1], b \in \mathbb{C}$, and $|b| \leq 1$, for any $Y, Y^{\prime} \in N_{R, x}$ and any multi-indices $\alpha, \alpha^{\prime}$ such that $|\alpha| \leq k,\left|\alpha^{\prime}\right| \leq k$, then

$$
\left|\frac{\partial^{|\alpha|+\left|\alpha^{\prime}\right|}}{\partial Y^{\alpha} \partial Y^{\prime \alpha^{\prime}}} P_{s}^{x, u, b}\left(Y, Y^{\prime}\right)\right| \leq C \exp \left(-C^{\prime}\left(|Y|^{2}+\left|Y^{\prime}\right|^{2}\right)\right) .
$$

Proof. If $B$ is reduced to a single point, (4.4) is a simple consequence of (4.3) and of the fact that if $p_{s}^{u}\left(x, x^{\prime}\right) \quad\left(x, x^{\prime} \in R\right)$ is the heat kernel associated to the operator $\Delta / 2-u|x|^{2} / 2$ with respect to the measure $d x^{\prime} / \sqrt{2 \pi}$, then by Mehler's formula [GIJ, Theorem 1.5.10]

$$
\begin{aligned}
& p_{s}^{u}\left(x, x^{\prime}\right) \\
& \quad=\frac{1}{\sqrt{s}} \sqrt{\frac{u^{1 / 2} s}{\sinh \left(u^{1 / 2} s\right)}} \exp \left(\frac{-u^{1 / 2}}{2 \sinh \left(u^{1 / 2} s\right)}\left[\cosh \left(u^{1 / 2} s\right)\left(x^{2}+x^{12}\right)-2 x x^{\prime}\right]\right) .
\end{aligned}
$$

Now let $Y_{s}(0 \leq s<+\infty)$ be a standard Brownian motion in $M_{R, x}$ starting at $Y_{0} \subset M_{R, x}$, let $E$ be the corresponding expectation operator, and let $U_{s}$ be the solution of the differential equation

$$
\begin{gathered}
\frac{d U_{s}}{d s}=U_{s}\left[-\sqrt{u} S-\frac{\sqrt{-1}}{\sqrt{2}} \sqrt{u} \hat{c}\left(\mathbf{J} A P^{L} Y_{s}\right)-\widehat{R^{N}}+b N_{H}\right], \\
U(0)=I_{\Lambda N_{R, x}^{*} \otimes_{R} \mathrm{C}} .
\end{gathered}
$$

In (4.6), $U_{s}$ is an even element of $\Lambda\left(T_{R}^{*} B\right) \hat{\otimes} \operatorname{End} \Lambda\left(\left(N_{R}^{*}\right) \otimes_{R} \mathbb{C}\right)_{x}$.

We claim that if $h$ is a bounded smooth section of $\Lambda\left(T_{R}^{*} B\right) \widehat{\otimes}\left(\Lambda\left(N_{R}^{*}\right) \otimes_{R} \mathbb{C}\right.$ on the fiber $M_{R, x}$, then

$$
\begin{aligned}
& \exp \left(-s \mathscr{B}_{u}^{2}+s b N_{H}\right) h\left(Y_{0}\right) \\
& \quad=E\left[\exp \left\{\frac{1}{2} \int_{0}^{s}\left\langle R^{M} Y, d Y\right\rangle-\frac{u}{2} \int_{0}^{s}\left|P^{N} Y_{t}\right|^{2} d t-\frac{1}{2} s \operatorname{Tr}\left[R^{M}\right]\right\} U_{s} h\left(Y_{s}\right)\right],
\end{aligned}
$$

where, in (4.7), $\int_{0}^{s}\left\langle R^{M} Y, d Y\right\rangle$ is a Stratonovitch stochastic integral [IkW, Chapter III; B6, §3] (which turns out to be an Ito stochastic integral since $R^{M}$ takes values in antisymmetric matrices). To prove (4.7), we first show that the right-hand side makes sense, i.e., the expression which appears under the expectation sign is integrable. In the sequel, the constants $C$ may vary from line to line. Clearly,

$$
\left|U_{s}\right| \leq C \exp \left(C(1+\sqrt{u}) s+C \int_{0}^{s}\left|P^{L} Y_{t}\right| d t\right) .
$$


Also we can write the expansion

$$
\exp \left\{\frac{1}{2} \int_{0}^{s}\left\langle R^{M} Y, d Y\right\rangle\right\}=1+\frac{1}{2} \int_{0}^{s} \frac{\left\langle R^{M} Y, d Y\right\rangle}{1 !}+\cdots ;
$$

the key point being that the right-hand side of (4.9) contains only a finite number of terms.

Now by [IMK, p. 27], for any $C>0$

$$
E\left[\exp \left(C \sup _{0 \leq t \leq 1}\left|P_{L} Y_{t}\right|\right)\right]<+\infty
$$

Also by [IMK, p. 27] and the Burkholder-Davis-Gundy inequalities [DeM, p. 304], the stochastic integral $\int_{0}^{s}\left\langle R^{M} Y, d Y\right\rangle$ lies in $\bigcap_{1 \leq p<+\infty} L_{p}$ (with respect to the Brownian measure). Using (4.10) and Hölder's inequality, it is now clear that the expectation in the right-hand side of (4.7) makes sense. Once this is proved, equality (4.7) is now a standard consequence of formula (3.35) for $\mathscr{B}_{u}^{2}$ and of Ito's formula, as used in [B8, Theorem 2.5].

Let $Q_{\left(Y, Y^{\prime}\right)}^{s}$ be the probability law on $\mathscr{C}\left([0, s] ; M_{R, x}\right)$ of the Brownian bridge $Y_{t}$ such that $Y_{0}=Y$ and $Y_{s}=Y^{\prime}$ [Si1, p. 40]. Then by disintegration of equality (4.7) as in [B1, Corollary of Theorem 4.2], we get

$$
\begin{aligned}
& P_{s}^{x, u, b}\left(Y, Y^{\prime}\right)=\frac{\exp \left(-\left|Y-Y^{\prime}\right|^{2} / 2 s\right)}{s^{m}} \\
& \quad \times E^{Q_{\left(Y, Y^{\prime}\right)}^{s}}\left[\exp \left\{\frac{1}{2} \int_{0}^{s}\left\langle R^{M} Y, d Y\right\rangle-\frac{u}{2} \int_{0}^{s}\left|P^{N} Y_{t}\right|^{2} d t-\frac{s}{2} \operatorname{Tr}\left[R^{M}\right]\right\} U_{s}\right] .
\end{aligned}
$$

Using (4.8), (4.11), and Hölder's inequality we find that

$$
\begin{aligned}
& \left|P_{s}^{x, u, b}\left(Y, Y^{\prime}\right)\right| \leq \frac{\exp \left(-\left|Y-Y^{\prime}\right|^{2} / 2 s\right)}{s^{m}} \\
& \times C\left\{E^{Q_{\left(Y, Y^{\prime}\right)}^{s}}\left[\left\|\exp \left\{\frac{1}{2} \int_{0}^{s}\left\langle R^{M} Y, d Y\right\rangle\right\}\right\|^{3}\right]\right. \\
& \times E^{Q_{\left(Y, Y^{\prime}\right)}^{s}}\left[\exp \left\{-\frac{3 u}{2} \int_{0}^{1}\left|P^{N} Y_{t}\right|^{2} d t\right\}\right] \\
& \left.\times E^{Q_{\left(Y, Y^{\prime}\right)}^{s}}\left[\exp \left\{3 C \int_{0}^{s}\left|P^{L} Y_{t}\right| d t\right\}\right]\right\}^{1 / 3} \exp (C(1+\sqrt{u}) s) \text {. }
\end{aligned}
$$

Let $w_{t}$ be a standard Brownian motion in $M_{R, x}$ such that $w_{0}=0$. Then by [Sil, p. 41], if $Y_{t}(0 \leq t \leq s)$ is the process

$$
Y_{t}=\left(1-\frac{t}{s}\right) Y+\frac{t}{s} Y^{\prime}+w_{t}-\frac{t}{s} w_{s}
$$


then the probability law of the process $Y_{t}(0 \leq t \leq s)$ is exactly $Q_{\left(Y, Y^{\prime}\right)}^{s}$. If $E$ now denotes expectation with respect to $w$, we deduce from (4.13) that

$$
\begin{aligned}
& E^{Q_{\left(Y, Y^{\prime}\right)}^{s}}\left[\left\|\exp \left\{\frac{1}{2} \int_{0}^{s}\left\langle R^{M} Y, d Y\right\rangle\right\}\right\|^{3}\right] \\
& =E\left[\| \exp \left\{\frac { 1 } { 2 } \int _ { 0 } ^ { s } \left\langleR^{M}\left(\left(1-\frac{t}{s}\right) Y+\frac{t}{s} Y^{\prime}+w_{t}-\frac{t}{s} w_{s}\right),\right.\right.\right. \\
& \left.\left.\left.\qquad\left(Y^{\prime}-Y-w_{s}\right) \frac{d t}{s}+d w_{t}\right\rangle\right\} \|^{3}\right] .
\end{aligned}
$$

We now expand the exponential appearing in the right-hand side of (4.14) in a finite power series as in (4.9). To dominate (4.14), we only need to bound a finite number of integrals $I_{s}^{p}$ (with $1 \leq p<+\infty$ ) given by

$$
\begin{aligned}
I_{s}^{p}=E\left[\| \int_{0}^{s}\left\langle R^{M}\left(\left(1-\frac{t}{s}\right) Y+\frac{t}{s} Y^{\prime}+w_{t}-\frac{t}{s} w_{s}\right)\right.\right. \\
\left.\left.\left(Y^{\prime}-Y-w_{s}\right) \frac{d t}{s}+d w_{t}\right\rangle \|^{p}\right] .
\end{aligned}
$$

Note that in the stochastic integral $\int_{0}^{s}\left\langle R^{M}(\cdots), d w_{t}\right\rangle$, the term $w_{s}$ can be factored out, so that the integral can be expressed by means of classical, i.e., nonanticipating, stochastic integrals [IkW, Chapters II and III].

We can then use the Burkholder-Davis-Gundy martingale inequalities [DeM, p. 304] and we easily obtain the bound

$$
\left|I_{s}^{p}\right| \leq C\left(1+|Y|^{2 p}+\left|Y^{\prime}\right|^{2 p}\right), \quad s \leq 1 .
$$

From (4.15) and (4.16) we deduce that there exist $C>0$ and $q \in \mathbb{N}$ such that if $s \leq 1$

$$
E^{Q_{\left(Y, Y^{\prime}\right)}^{s}}\left[\left\|\exp \left\{\frac{1}{2} \int_{0}^{s}\left\langle R^{M} Y, d Y\right\rangle\right\}\right\|^{3}\right] \leq C\left(1+|Y|^{q}+\left|Y^{\prime}\right|^{q}\right) .
$$

Also if $Y, Y^{\prime} \in N_{R}$, under $Q_{\left(Y, Y^{\prime}\right)}^{s}, P^{L} Y$ is a standard Brownian bridge in $L_{R}$ such that $P^{L} Y_{0}=P^{L} Y_{s}=0$. Therefore by [IMK, p. 27] there exists $M>0$ such that if $Y \in N_{R, x}$ and $s \in[0,1]$

$$
E^{Q_{\left(Y, Y^{\prime}\right)}^{s}}\left[\exp \left(3 C \int_{0}^{s}\left|P^{L} Y_{t}\right| d t\right)\right] \leq M
$$

By (4.5) and (4.11), we know that if $Y, Y^{\prime} \in N_{R, x}$, then

$$
\begin{aligned}
& E^{Q_{\left(Y, Y^{\prime}\right)}^{s}}\left[\exp \left\{\frac{-3 u}{2} \int_{0}^{s}\left|P^{N} Y_{t}\right|^{2} d t\right\}\right] \\
& \quad=\left(\frac{(3 u)^{1 / 2} s}{\sinh (3 u)^{1 / 2} s}\right)^{n} \\
& \quad \times \exp \left\{\frac{\left|Y-Y^{\prime}\right|^{2}}{2 s}-\frac{(3 u)^{1 / 2}}{2 \sinh \left((3 u)^{1 / 2} s\right)}\left[\cosh \left((3 u)^{1 / 2} s\right)\left(|Y|^{2}+\left|Y^{\prime}\right|^{2}\right)-2\left\langle Y, Y^{\prime}\right\rangle\right]\right\} .
\end{aligned}
$$


From (4.19), we deduce that there are $C, C^{\prime}>0$ such that if $u, s, Y$, and $Y^{\prime}$ are chosen as indicated in the statement of our theorem, then

$$
\begin{aligned}
& \exp \left(-\frac{\left|Y-Y^{\prime}\right|^{2}}{2 s}\right)\left\{E^{Q_{\left(Y, Y^{\prime}\right)}^{s}} \exp \left(\frac{-3 u}{2} \int_{0}^{s}\left|P^{N} Y\right|^{2} d t\right)\right\}^{1 / 3} \\
& \quad \leq C \exp \left(-C^{\prime}\left(|Y|^{2}+\left|Y^{\prime}\right|^{2}\right)\right) .
\end{aligned}
$$

When $\alpha, \alpha^{\prime}=0$, the bound (4.4) now follows from (4.12), (4.17), (4.18), and (4.20). For general $\alpha, \alpha^{\prime}$ one can easily use the Malliavin calculus [Ma, B7], to obtain the bound (4.4). This is especially easy here, since we work only with the flat Brownian motion. Details are left to the reader.

Another method of proof of Theorem 4.1 is to start from Mehler's formula (4.5) and to use Duhamel's formula to estimate the kernel $P_{s}^{x, u, b}$ and its derivatives.

(c) Translation invariance of the supertrace of the heat kernel. For any $Y \in M_{R, x}$ and $s>0$,

$$
P_{s}^{x, u, b}(Y, Y) \in\left(\Lambda\left(T_{R}^{*} B\right) \widehat{\otimes} \operatorname{End}\left(\Lambda\left(N_{R}^{*}\right)\right)\right)_{x}^{\text {even }} .
$$

Therefore, if we use the conventions in Quillen [Q1], the supertrace $\operatorname{Tr}_{s}\left[P_{s}^{x, u, b}(Y, Y)\right]$ lies in $\left(\Lambda^{\text {even }}\left(T_{R}^{*} B\right)\right)_{x}$. If $Y_{0} \in L_{R, x}$, we define $c\left(A Y_{0}\right)$ as in (3.34).

We now prove a key translation invariance property of $\operatorname{Tr}_{s}\left[P_{s}^{x, u, b}(Y, Y)\right]$.

Theorem 4.2. For any $u>0, s>0, x \in B, b \in \mathbb{C}, Y, Y^{\prime} \in M_{R, x}$, and $Y_{0} \in L_{R, x}$, we have

$$
\begin{gathered}
P_{s}^{x, u, b}\left(Y+Y_{0}, Y^{\prime}+Y_{0}\right)=\exp \left(-\frac{c\left(A Y_{0}\right)}{\sqrt{2}}-\frac{\left\langle R^{M} Y_{0}, Y\right\rangle}{2}\right), \\
P_{s}^{x, u, b}\left(Y, Y^{\prime}\right) \exp \left(\frac{c\left(A Y_{0}\right)}{\sqrt{2}}+\frac{\left\langle R^{M} Y_{0}, Y^{\prime}\right\rangle}{2}\right) .
\end{gathered}
$$

In particular, for any $u>0, s>0, x \in B, b \in \mathbb{C}, Y \in M_{R, x}$, and $Y_{0} \in L_{R, x}$

$$
\begin{aligned}
\operatorname{Tr}_{s}\left[P_{s}^{x, u, b}(Y, Y)\right] & =\operatorname{Tr}_{s}\left[P_{s}^{x, u, b}\left(Y+Y_{0}, Y+Y_{0}\right)\right], \\
\operatorname{Tr}_{s}\left[N_{H} P_{s}^{x, u, b}(Y, Y)\right] & =\operatorname{Tr}_{s}\left[N_{H} P_{s}^{x, u, b}\left(Y+Y_{0}, Y+Y_{0}\right)\right] .
\end{aligned}
$$

Proof. As is obvious from Theorem 3.10, the operator $\mathscr{B}_{u}^{2}$ is not invariant under translations by elements of $L_{R}$. So (4.21) is a nontrivial statement.

Let $e_{1}, \ldots, e_{2 m}$ be an orthonormal base of $M_{R}$ such that $e_{1}, \ldots, e_{2 n}$ is an orthonormal base of $N_{R}$ and $e_{2 n+1}, \ldots, e_{2 m}$ is an orthonormal base of $L_{R}$. We also take $f_{1}, \ldots, f_{2 k}$ and $f^{1}, \ldots, f^{2 k}$ as in (3.34). Then

$$
c\left(A Y_{0}\right)=-\sum_{1}^{2 k} f^{j} c\left(A\left(f_{j}\right) Y_{0}\right) .
$$


Clearly,

$$
\left[c\left(A Y_{0}\right), c\left(e_{i}\right)\right]=2\left\langle A Y_{0}, e_{i}\right\rangle, \quad\left[c\left(A Y_{0}\right), \hat{c}\left(e_{i}\right)\right]=0 .
$$

Using formula (3.35) for $S$, we deduce from (4.24) that since $A Y_{0}$ takes values in $N_{R}$

$$
\left[c\left(A Y_{0}\right), S\right]=-\sqrt{-1} \hat{c}\left(\mathbf{J} A Y_{0}\right) .
$$

From (4.24) and (4.25), we get

$$
\left[c\left(A Y_{0}\right),\left[c\left(A Y_{0}\right), S\right]\right]=0 .
$$

Using (4.25) and (4.26), we find that

$$
\exp \left(\frac{-c\left(A Y_{0}\right)}{\sqrt{2}}\right) S \exp \left(\frac{c\left(A Y_{0}\right)}{\sqrt{2}}\right)=S+\sqrt{-1} \frac{\hat{c}\left(\mathbf{J} A Y_{0}\right)}{\sqrt{2}}
$$

Also

$$
\begin{aligned}
& \exp \left(-\frac{\left\langle R^{M} Y_{0}, Y\right\rangle}{2}\right)\left(\nabla_{e_{i}}+\frac{1}{2}\left\langle R^{M} Y, e_{i}\right\rangle\right) \exp \left(\frac{\left\langle R^{M} Y_{0}, Y\right\rangle}{2}\right) \\
& =\nabla_{e_{i}}+\frac{1}{2}\left\langle R^{M}\left(Y+Y_{0}\right), e_{i}\right\rangle
\end{aligned}
$$

Let $\tau_{Y_{0}}$ be the map

$$
h \in \mathcal{J} \rightarrow h\left(\cdot+Y_{0}\right) \in \mathscr{J} .
$$

Set

$$
\mathscr{B}_{u, Y_{0}}^{2}=\tau_{Y_{0}} \mathscr{B}_{u}^{2} \tau_{-Y_{0}}
$$

Using Theorem 3.10, (4.27) and (4.28), we get

$$
\begin{aligned}
\mathscr{B}_{u, Y_{0}}^{2}-b N_{H}= & \exp \left(-\frac{c\left(A Y_{0}\right)}{\sqrt{2}}-\frac{\left\langle R^{M} Y_{0}, Y\right\rangle}{2}\right)\left(\mathscr{B}_{u}^{2}-b N_{H}\right) \\
& \times \exp \left(\frac{c\left(A Y_{0}\right)}{\sqrt{2}}+\frac{\left\langle R^{M} Y_{0}, Y\right\rangle}{2}\right) .
\end{aligned}
$$

From (4.31), we immediately deduce (4.21). By [Q1], supertraces vanish on supercommutators. (4.22) then follows from (4.21).

Remark 4.3. Theorem 4.2 exactly says that the function

$$
Y \in M_{R, x} \rightarrow \operatorname{Tr}_{s}\left[P_{s}^{x, u, b}(Y, Y)\right] \in \Lambda^{\text {even }}\left(T_{R}^{*} B\right)_{x}
$$

factors through the normal bundle $N_{R}$, i.e., $\operatorname{Tr}_{s}\left[P_{s}^{x, u, b}(Y, Y)\right]$ depends only on $j(Y) \in N_{R, x}$.

(d) A generalized supertrace. For $s>0$, the operators $\exp \left(-s \mathscr{B}_{u}^{2}+s b N_{H}\right)$ and $N_{H} \exp \left(-s \mathscr{B}_{u}^{2}+s b N_{H}\right)$ are in general not trace class. This is made especially obvious by Theorem 4.2 , which indicates that $\operatorname{Tr}_{s}\left[P_{s}^{x, u, b}(Y, Y)\right]$ and $\operatorname{Tr}_{s}\left[N_{H} P_{s}^{x, u, b}(Y, Y)\right]$ are invariant by translations in $L_{R}$.

Still, using Theorems 4.1 and 4.2, we can define generalized supertraces of $\exp \left(-s \mathscr{B}_{u}^{2}+s b N_{H}\right)$ and $N_{H} \exp \left(-s \mathscr{B}_{u}^{2}+s b N_{H}\right)$. 
Definition 4.4. For $s>0, u>0$, and $b \in \mathbb{C}$ we define the generalized supertraces $\operatorname{Tr}_{s}\left[\exp \left(-s \mathscr{B}_{u}^{2}+s b N_{H}\right)\right]$ and $\operatorname{Tr}_{s}\left[N_{H} \exp \left(-s \mathscr{B}_{u}^{2}+s b N_{H}\right)\right]$ by the formulas

$$
\begin{aligned}
\operatorname{Tr}_{s}\left[\exp \left(-s \mathscr{B}_{u}^{2}+s b N_{H}\right)\right] & =\int_{N_{R, x}} \operatorname{Tr}_{s}\left[P_{s}^{x, u, b}(Y, Y)\right] \frac{d v_{N}(Y)}{(2 \pi)^{n}} \\
\operatorname{Tr}_{s}\left[N_{H} \exp \left(-s \mathscr{B}_{u}^{2}+s b N_{H}\right)\right] & =\int_{N_{R, x}} \operatorname{Tr}_{s}\left[N_{H} P_{s}^{x, u, b}(Y, Y)\right] \frac{d v_{N}(Y)}{(2 \pi)^{n}} .
\end{aligned}
$$

$\operatorname{Tr}_{s}\left[\exp \left(-s \mathscr{B}_{u}^{2}+s b N_{H}\right)\right]$ and $\operatorname{Tr}_{s}\left[N_{H} \exp \left(-s \mathscr{B}_{u}^{2}+s b N_{H}\right)\right]$ are smooth sections of $\Lambda^{\text {even }}\left(T_{R}^{*} B\right)$.

In the sequel, we use the notation

$$
P_{s}^{x, u}\left(Y, Y^{\prime}\right)=P_{s}^{x, u, 0}\left(Y, Y^{\prime}\right) \text {. }
$$

Also, using the bounds (4.4), one easily verifies that $P_{s}^{x, u, b}\left(Y, Y^{\prime}\right)$ depends smoothly on $b \in \mathbb{C}$. Set

$$
Q_{s}^{x, u}\left(Y, Y^{\prime}\right)=\left[\frac{\partial P_{s}^{x, u, b}}{\partial b}\left(Y, Y^{\prime}\right)\right]_{b=0} .
$$

By using bounds similar to (4.4), one easily verifies that the form $\operatorname{Tr}_{s}\left[\exp \left(-s \mathscr{B}_{u}^{2}+s b N_{H}\right)\right]$ depends holomorphically on $b \in \mathbb{C}$, and that

$$
\begin{aligned}
\frac{\partial}{\partial b}\left\{\operatorname{Tr}_{s}\left[\exp \left(-s \mathscr{B}_{u}^{2}+s b N_{H}\right)\right]\right\}_{b=0} \\
\quad=\int_{N_{R, x}} \operatorname{Tr}_{s}\left[Q_{s}^{x, u}(Y, Y)\right] \frac{d v_{N}(Y)}{(2 \pi)^{n}} .
\end{aligned}
$$

(e) A double transgression formula for generalized supertraces. We establish here a double transgression formula which can be considered as an analogue for generalized supertraces of formulas in [BGS1, Theorem 1.15 and BGS2, Theorem 2.9], which were established for genuine finite- or infinite-dimensional supertraces.

Definition 4.5. $P^{B}$ denotes the vector space of smooth differential forms on $B$ which are sums of forms of type $(p, p) . P^{B, 0}$ is the subset of $\omega \in P^{B}$ such that there exist smooth forms $\alpha, \beta$ for which $\omega=\partial \alpha+\bar{\partial} \beta$.

Theorem 4.6. For any $b \in \mathbb{C}$ and $u>0$, the forms $\operatorname{Tr}_{s}\left[\exp \left(-\mathscr{B}_{u}^{2}+b N_{H}\right)\right]$ and $\operatorname{Tr}_{s}\left[N_{H} \exp \left(-\mathscr{B}_{u}^{2}+b N_{H}\right)\right]$ lie in $P^{B}$. The forms $\operatorname{Tr}_{s}\left[\exp \left(-\mathscr{B}_{u}^{2}\right)\right]$ are closed and their cohomology class does not depend on $u>0$. More precisely,

$$
\frac{\partial}{\partial u} \operatorname{Tr}_{s}\left[\exp \left(-\mathscr{B}_{u}^{2}\right)\right]=\frac{\bar{\partial} \partial}{u}\left\{\frac{\partial}{\partial b} \operatorname{Tr}_{s}\left[\exp \left(-\mathscr{B}_{u}^{2}+b N_{H}\right)\right]_{b=0}\right\}
$$

Finally,

$$
\operatorname{Tr}_{s}\left[N_{H} \exp \left(-\mathscr{B}_{u}^{2}\right)\right]=\frac{\partial}{\partial b}\left\{\operatorname{Tr}_{s}\left[\exp \left(-\mathscr{B}_{u}^{2}+b N_{H}\right)\right]\right\}_{b=0}
$$


Proof. To prove that the forms

$$
\operatorname{Tr}_{s}\left[\exp \left(-\mathscr{B}_{u}^{2}+b N_{H}\right)\right] \text { and } \operatorname{Tr}_{s}\left[N_{H} \exp \left(-\mathscr{B}_{u}^{2}+b N_{H}\right)\right]
$$

lie in $P^{B}$, we proceed as in [BGS1, Theorem 1.9]. Remember that we grade the algebra $\Lambda\left(N_{R}^{*}\right) \otimes_{R} \mathbb{C}=\Lambda\left(\bar{N}^{*}\right) \widehat{\otimes} \Lambda\left(N^{*}\right)$ by the operator $N_{V}-N_{H}$. Clearly,

$$
\sqrt{-1} \frac{\hat{c}\left(\mathbf{J} A P^{L} Y\right)}{\sqrt{2}}=\sqrt{-1}\left(i_{A P^{L} y}-i_{A P^{L} y}^{*}\right) .
$$

As an element of $\Lambda^{1}\left(T_{R}^{*} B\right) \hat{\otimes} \operatorname{Hom}(L, N), A$ is of complex type $(1,0)$. So $i_{A P^{L} y} \in \Lambda^{1}\left(T_{R}^{*} B\right) \widehat{\otimes} \operatorname{End}\left(\Lambda\left(N_{R}^{*}\right) \otimes_{R} \mathbb{C}\right)$ is of complex type $(1,0)$ and decreases the total degree in $\Lambda\left(N_{R}^{*}\right) \otimes_{R} \mathbb{C}$ by one. Similarly $i_{A P^{L} y}^{*}$ is a one-form of type $(0,1)$ and increases the total degree in $\Lambda\left(N_{R}^{*}\right) \otimes_{R} \mathbb{C}$ by one. Also in formula (3.36) for $\mathscr{B}_{u}^{2}$, the terms other than $(\sqrt{-1} \sqrt{u} / \sqrt{2}) \hat{c}\left(\mathbf{J} A P^{L} Y\right)$ are products of zero or $(1,1)$ forms with operators which preserve the total grading in $\Lambda\left(N_{R}^{*}\right) \otimes_{R} \mathbb{C}$. By proceeding as in [BGS1, Proposition 1.8 and Theorem 1.9], it is now clear that $\operatorname{Tr}_{s}\left[P_{s}^{x, u, b}(Y, Y)\right]$ and $\operatorname{Tr}_{s}\left[N_{H} P_{s}^{x, u, b}(Y, Y)\right]$ are sums of forms of complex type $(p, p)$. Therefore the forms $\operatorname{Tr}_{s}\left[\exp \left(-\mathscr{B}_{u}{ }^{2}+b N_{H}\right)\right]$ and $\operatorname{Tr}_{s}\left[N_{H} \exp \left(-\mathscr{B}_{u}^{2}+b N_{H}\right)\right]$ lie in $P^{B}$.

We now prove that the forms $\operatorname{Tr}_{s}\left[\exp \left(-\mathscr{B}_{u}^{2}\right)\right]$ are closed. First, let us point out that Quillen's superconnection formalism [Q1] cannot be used (even in an infinite-dimensional context) as in [B1, Theorems 2.6 and 3.6], since the forms $\operatorname{Tr}_{s}\left[\exp \left(-\mathscr{B}_{u}^{2}\right)\right]$ are only generalized supertraces. Still, we will mimick the strategy in [B1, Theorem 2.6].

Take $x \in B$, and let $\mathscr{V}$ be an open neighborhood of $x \in B$, which is holomorphically equivalent to an open ball in $\mathbb{C}^{k}$, with 0 representing $x$. In particular, the exterior algebra $\Lambda\left(T_{R}^{*} B\right)$ is canonically trivialized on $\mathscr{V}$. Let $l \in R \rightarrow x_{l} \in \mathbb{C}^{k}$ be a straight line, with $x_{0}=x$. We trivialize the vector bundle $M$ along the line $x_{l}$ by parallel transport with respect to the connection ${ }^{0} \nabla^{M}$, so that $M_{x_{1}}$ is identified with $M_{x}$. Note that this trivialization preserves the splitting $M=L \oplus N$. Similarly we trivialize the vector bundle $\mathscr{J}$ along the line $x_{l}$ using the connection ${ }^{0} \widetilde{\nabla}^{J}$, so that $\mathscr{J}_{x_{l}}$ is identified with $\mathscr{J}_{x}$. The operators $\mathscr{B}_{u, x_{l}}^{2}$ and $\exp \left(-\mathscr{B}_{u, x_{l}}^{2}+b N_{H}\right)$ now lie in $\left(\Lambda\left(T_{R}^{*} B\right) \hat{\otimes} \text { End } \mathscr{J}\right)_{x}^{\text {even }}$ and so act on the fixed vector space $\mathscr{J}_{x}$.

We will evaluate the kernels $P_{s}^{x_{1}, u}$ with respect to the volume element $d v_{M_{x}} /(2 \pi)^{m}$ in the fiber $M_{x}$. By using Duhamel's formula as in [B1, Proposition 2.8], we find easily that

$$
\begin{aligned}
\frac{\partial}{\partial l} P_{s}^{x_{l}, u}(Y, Y)= & -\int_{0}^{1} d s \int_{M_{R_{X}}} P_{s}^{x_{l}, u}\left(Y, Y^{\prime}\right), \\
& \frac{\partial \mathscr{B}_{u, x_{l}}^{2}}{\partial l} P_{1-s}^{x_{l}, u}\left(Y^{\prime}, Y\right) \frac{d v_{M}\left(Y^{\prime}\right)}{(2 \pi)^{m}} .
\end{aligned}
$$


The rigorous derivation of (4.38) requires estimates on $P_{s}^{u, x_{t}}$ and on its derivatives which say that for any given $M, 0<M<+\infty$,

$$
\begin{aligned}
& \left|\frac{\partial^{|\alpha|+\left|\alpha^{\prime}\right|}}{\partial Y^{\alpha} \partial Y^{\prime \alpha^{\prime}}} P_{s}^{x_{l}, u}\left(Y, Y^{\prime}\right)\right| \\
& \quad \leq \frac{C}{s^{h\left(\alpha, \alpha^{\prime}\right)}} \exp \left(\frac{-\left|Y-Y^{\prime}\right|^{2}}{4 s}\right), \quad|Y| \leq M \text { or }\left|Y^{\prime}\right| \leq M,
\end{aligned}
$$

which easily follows from (4.12), (4.17), and (4.18) when $\alpha, \alpha^{\prime}=0$, and from the Malliavin calculus [Ma, B7] for general $\alpha, \alpha^{\prime}$. Estimate (4.39) is needed to make sense of the integration in (4.38) on the noncompact fiber $M_{R, x}$.

Using (4.38) and the essential fact that the connection ${ }^{0} \nabla^{M}$ preserves the splitting $M=L \oplus N$, we deduce that

$$
\begin{aligned}
& d \operatorname{Tr}_{s}\left[\exp \left(-\mathscr{B}_{u}^{2}\right)\right] \\
& =-\int_{0}^{1} d s \int_{M_{R_{x}} \times N_{R_{x}}} \operatorname{Tr}_{s}\left\{P_{s}^{x, u}\left(Y, Y^{\prime}\right)\left[^{0} \widetilde{\nabla}^{\mathcal{g}}, \mathscr{B}_{u}^{2}\right] P_{1-s}^{x, u}\left(Y^{\prime}, Y\right)\right\} \frac{d v_{M}\left(Y^{\prime}\right)}{(2 \pi)^{m}} \frac{d v_{N}(Y)}{(2 \pi)^{n}} .
\end{aligned}
$$

By Theorem 3.16, we know that

$$
\left[{ }^{0} \widetilde{\nabla}^{\mathcal{G}}, \mathscr{B}_{u}^{2}\right]=\left[\nabla_{A Y}-D^{N}-\sqrt{u} V\left(P^{N} Y\right)+\frac{c\left(P^{N} R^{M} Y\right)}{2 \sqrt{2}}, \mathscr{B}_{u}^{2}\right]
$$

Remember that $A P^{L} Y$ is a one-form with values in $N_{R}$. The operator $\mathscr{L}_{u}$ given by

$$
\mathscr{L}_{u}=\nabla_{A P^{L} Y}-D^{N}-\sqrt{u} V\left(P^{N} Y\right)+\frac{c\left(P^{N} R^{M} Y\right)}{2 \sqrt{2}}
$$

is an operator of order one, which involves only differentiation in the directions of $N_{R, x}$. By proceeding as in [B1, Proposition 2.8], while making the obvious changes, we find that

$$
\left[\mathscr{L}_{u}, P_{1}^{x, u}\right](Y, Y)=-\int_{0}^{1} d s \int_{M_{R_{x}}} P_{s}^{x, u}\left(Y, Y^{\prime}\right)\left[\mathscr{L}_{u}, \mathscr{B}_{u}^{2}\right] P_{1-s}^{x, u}\left(Y^{\prime}, Y\right) \frac{d v_{M}\left(Y^{\prime}\right)}{(2 \pi)^{m}}
$$

Let $K\left(Y, Y^{\prime}\right) \quad\left(Y, Y^{\prime} \in N_{R, x}\right)$ be any smooth kernel on $N_{R, x}$, with $K\left(Y, Y^{\prime}\right) \in \Lambda\left(T_{R}^{*} B\right) \widehat{\otimes} \operatorname{End}\left(\Lambda\left(N_{R}^{*}\right) \otimes_{R} \mathbb{C}\right)$, which has compact support, i.e., it vanishes for $|Y|+\left|Y^{\prime}\right|$ large enough. Let ${ }^{N} P_{s}^{x, u}\left(Y, Y^{\prime}\right) \quad\left(Y, Y^{\prime} \in N_{R, x}\right)$ be 
the restriction of the kernel $P_{s}^{x, u}\left(Y, Y^{\prime}\right)$ to $N_{R, x}$. Then (4.44)

$$
\begin{aligned}
& \int_{N_{R_{x}}} \operatorname{Tr}_{s}\left\{\left({ }^{N} P_{1}^{x, u} K-K{ }^{N} P_{1}^{x, u}\right)(Y, Y)\right\} \frac{d v_{N}(Y)}{(2 \pi)^{n}} \\
& =\int_{N_{R_{x}} \times N_{R_{x}}} \operatorname{Tr}_{s}\left\{P_{1}^{x, u}\left(Y, Y^{\prime}\right) K\left(Y^{\prime}, Y\right)-K\left(Y, Y^{\prime}\right) P_{1}^{x, u}\left(Y^{\prime}, Y\right)\right\} \frac{d v_{N}(Y)}{(2 \pi)^{n}} \frac{d v_{N}\left(Y^{\prime}\right)}{(2 \pi)^{n}} \\
& =\int_{N_{R_{x}} \times N_{R_{x}}} \operatorname{Tr}_{s}\left\{\left[P_{1}^{x, u}\left(Y, Y^{\prime}\right), K\left(Y^{\prime}, Y\right)\right]\right\} \frac{d v_{N}(Y)}{(2 \pi)^{n}} \frac{d v_{N}\left(Y^{\prime}\right)}{(2 \pi)^{n}} .
\end{aligned}
$$

By [Q1], we know that

$$
\operatorname{Tr}_{s}\left\{\left[P_{1}^{x, u}\left(Y, Y^{\prime}\right), K\left(Y^{\prime}, Y\right)\right]\right\}=0 .
$$

From (4.44) and (4.45), we get

$$
\int_{N_{R_{x}}} \operatorname{Tr}_{s}\left\{\left[{ }^{N} P_{1}^{x, u}, K\right](Y, Y)\right\} \frac{d v_{N}(Y)}{(2 \pi)^{n}}=0 .
$$

Since the vector field $A P^{L} Y$ is divergence free, one verifies that for any $Y \in$ $N_{R, x},\left[\mathscr{L}_{u}, P_{1}^{x, u}\right](Y, Y)=\left[\mathscr{L}_{u},{ }^{N} P_{1}^{x, u}\right](Y, Y)$. By approximating the operator $\mathscr{L}_{u}$ by smooth kernels $K$ on $N_{R, x}$ and taking into account the uniform bounds (4.4), we deduce from (4.46) that

$$
\int_{N_{R_{x}}} \operatorname{Tr}_{s}\left\{\left[\mathscr{L}_{u}, P_{1}^{x, u}\right](Y, Y)\right\} \frac{d v_{N}(Y)}{(2 \pi)^{n}}=0 .
$$

$A P^{N} Y$ is a one-form on $B$ with values in $L_{R}$. By Theorem 4.2, we know that $\operatorname{Tr}_{s}\left[P_{1}^{x, u}(Y, Y)\right]$ is invariant under translations by elements of $L_{R}$. Also, it is clear that since $A P^{N} Y$ is a one-form taking values in the set of divergence free vector fields on $M_{R}$, then (4.48)

$$
\begin{aligned}
& \nabla_{A P^{N} Y} \operatorname{Tr}_{s}\left[P_{1}^{x, u}(Y, Y)\right] \\
& =-\int_{0}^{1} d s \int_{M_{R_{x}}} \operatorname{Tr}_{s}\left\{P_{s}^{x, u}\left(Y, Y^{\prime}\right)\left[\nabla_{A P^{N} Y^{\prime}}, \mathscr{B}_{u}^{2}\right] P_{1-s}^{x, u}\left(Y^{\prime}, Y\right)\right\} \frac{d v_{M}\left(Y^{\prime}\right)}{(2 \pi)^{m}} .
\end{aligned}
$$

Therefore both sides of (4.48) vanish identically. Using this fact together with (4.40), (4.41), and (4.47), we get

$$
d \operatorname{Tr}_{s}\left[\exp \left(-\mathscr{B}_{u}^{2}\right)\right]=0
$$

We now replace the manifold $B$ by the manifold $B \times R_{+}^{*}$. The vector bundles $L, M$, and $N$ lift trivially to $B \times R_{+}^{*}$. The superconnection $\mathscr{B}_{u}$ is replaced by $\mathscr{B}_{u}^{\prime}$ given by

$$
\mathscr{B}_{u}^{\prime}=\mathscr{B}_{u}+d u \frac{\partial}{\partial u}
$$


Clearly,

$$
\left(\mathscr{B}_{u}^{\prime}\right)^{2}=\mathscr{B}_{u}^{2}+d u\left(\frac{\partial}{\partial u} \mathscr{B}_{u}\right)
$$

Although $B \times R_{+}^{*}$ is not a complex manifold, we can easily reproduce what has been done in (4.38)-(4.49) for the manifold $B$. In particular, the form $\omega_{u}$ on $B \times R_{+}^{*}$ given by

$$
\omega_{u}=\operatorname{Tr}_{s}\left[\exp \left(-\mathscr{B}_{u}^{2}-d u \frac{\partial}{\partial u} \mathscr{B}_{u}\right)\right]
$$

is closed. $\omega_{u}$ can be written in the form

$$
\omega_{u}=\operatorname{Tr}_{s}\left[\exp \left(-\mathscr{B}_{u}^{2}\right)\right]+d u \alpha_{u}
$$

Since $\omega_{u}$ is closed, we deduce from (4.53) that

$$
\frac{\partial}{\partial u} \operatorname{Tr}_{s}\left[\exp \left(-\mathscr{B}_{u}^{2}\right)\right]=d \alpha_{u} .
$$

From (4.54), we find that the cohomology class of the forms $\operatorname{Tr}_{s}\left[\exp \left(-\mathscr{B}_{u}^{2}\right)\right]$ does not depend on $u>0$. The form $\alpha_{u}$ can be calculated using Duhamel's formula. We get

$$
\alpha_{u}=-\int_{0}^{1} d s \int_{M_{R_{x}} \times N_{R_{x}}} \operatorname{Tr}_{s}\left[P_{s}^{x, u}\left(Y, Y^{\prime}\right) \frac{\partial \mathscr{B}_{u}}{\partial u} P_{1-s}^{x, u}\left(Y^{\prime}, Y\right)\right] \frac{d v_{M}\left(Y^{\prime}\right)}{(2 \pi)^{m}} \frac{d v_{N}(Y)}{(2 \pi)^{n}}
$$

Equivalently,

$$
\begin{array}{r}
\alpha_{u}=\frac{-1}{2 \sqrt{u}} \int_{0}^{1} d s \int_{M_{R_{x}} \times N_{R_{x}}} \operatorname{Tr}_{s}\left[P_{s}^{x, u}\left(Y, Y^{\prime}\right) \sqrt{-1}\left(i_{P^{N} y^{\prime}}-i_{P^{N} y^{\prime}}^{*}\right) P_{1-s}^{x, u}\left(Y^{\prime}, Y\right)\right] \\
\frac{d v_{M}\left(Y^{\prime}\right)}{(2 \pi)^{m}} \frac{d v_{N}(Y)}{(2 \pi)^{n}} .
\end{array}
$$

We now prove (4.36). By proceeding as in (4.40), we find that

$$
\begin{aligned}
& d \operatorname{Tr}_{s}\left[\exp \left(-\mathscr{B}_{u}^{2}+b N_{H}\right)\right] \\
&=-\int_{0}^{1} d s \int_{M_{R_{x}} \times N_{R_{x}}} \operatorname{Tr}_{s}\left[P_{s}^{x, u, b}\left(Y, Y^{\prime}\right)\left[\nabla^{\mathcal{J}}, \mathscr{B}_{u}^{2}\right] P_{1-s}^{x, u, b}\left(Y^{\prime}, Y\right)\right] \\
& \frac{d v_{M}\left(Y^{\prime}\right)}{(2 \pi)^{m}} \frac{d v_{N}(Y)}{(2 \pi)^{n}}
\end{aligned}
$$

We rewrite $(4.41)$ in the form

$$
\begin{aligned}
{\left[{ }^{0} \nabla^{\mathscr{J}}, \mathscr{B}_{u}^{2}\right]=} & {\left[\nabla_{A Y}-D^{N}-\sqrt{u} V\left(P^{N} Y\right)+\frac{c\left(P^{N} R^{M} Y\right)}{2 \sqrt{2}}, \mathscr{B}_{u}^{2}-b N_{H}\right] } \\
& -b \sqrt{u}\left[V\left(P^{N} Y\right), N_{H}\right] .
\end{aligned}
$$


By using the same arguments as in (4.41)-(4.49), we then deduce from (4.57) and (4.58) that

$$
\begin{aligned}
d \operatorname{Tr}_{s} & {\left[\exp \left(-\mathscr{B}_{u}^{2}+b N_{H}\right)\right] } \\
=\int_{0}^{1} d s \int_{M_{R_{x}} \times N_{R_{x}}} \operatorname{Tr}_{s}\left[P_{s}^{x, u, b}\left(Y, Y^{\prime}\right) b \sqrt{u}\left[V\left(P^{N} Y^{\prime}\right), N_{H}\right] P_{1-s}^{x, u, b}\left(Y^{\prime}, Y\right)\right] & \frac{d v_{M}\left(Y^{\prime}\right)}{(2 \pi)^{m}} \frac{d v_{N}(Y)}{(2 \pi)^{n}} .
\end{aligned}
$$

Clearly,

$$
\left[V\left(P^{N} Y^{\prime}\right), N_{H}\right]=\sqrt{-1}\left(i_{P^{N} y^{\prime}}+i_{P^{N} y^{\prime}}^{*}\right) .
$$

Using (4.59) and (4.60), we get

$$
\begin{aligned}
& d \frac{\partial}{\partial b}\left\{\operatorname{Tr}_{s}\left[\exp \left(-\mathscr{B}_{u}^{2}+b N_{H}\right)\right]\right\}_{b=0} \\
&=\int_{0}^{1} d s \int_{M_{R_{x}} \times N_{R_{x}}} \operatorname{Tr}_{s}\left[P_{s}^{x, u}\left(Y, Y^{\prime}\right) \sqrt{-u}\left(i_{P^{N} y^{\prime}}+i_{P^{N} y^{\prime}}^{*}\right) P_{1-s}^{x, u}\left(Y^{\prime}, Y\right)\right] \\
& \frac{d v_{M}\left(Y^{\prime}\right)}{(2 \pi)^{m}} \frac{d v_{N}(Y)}{(2 \pi)^{n}}
\end{aligned}
$$

By using the same arguments as in [BGS1, Theorem 1.9] (which we already used at the beginning of our proof), we can decompose (4.61) by complex type. We get

$$
\begin{aligned}
& \partial \frac{\partial}{\partial b}\left\{\operatorname{Tr}_{s}\left[\exp \left(-\mathscr{B}_{u}^{2}+b N_{H}\right)\right]\right\}_{b=0} \\
& =\sqrt{u} \int_{0}^{1} d s \int_{M_{R_{x}} \times N_{R_{x}}} \operatorname{Tr}_{s}\left[P_{s}^{x, u}\left(Y, Y^{\prime}\right) \sqrt{-1} i_{P^{N} y^{\prime}}^{*} P_{1, s}^{x, u}\left(Y^{\prime}, Y\right)\right] \frac{d v_{M}\left(Y^{\prime}\right)}{(2 \pi)^{m}} \frac{d v_{N}(Y)}{(2 \pi)^{n}}, \\
& \bar{\partial} \frac{\partial}{\partial b}\left\{\operatorname{Tr}_{s}\left[\exp \left(-\mathscr{B}_{u}^{2}+b N_{H}\right)\right]\right\}_{b=0} \\
& =\sqrt{u} \int_{0}^{1} d s \int_{M_{R_{x}} \times N_{R_{x}}} \operatorname{Tr}_{s}\left[P_{s}^{x, u}\left(Y, Y^{\prime}\right) \sqrt{-1} i_{P^{N} y^{\prime}} P_{1-s}^{x, u}\left(Y^{\prime}, Y\right)\right] \frac{d v_{M}\left(Y^{\prime}\right)}{(2 \pi)^{m}} \frac{d v_{N}(Y)}{(2 \pi)^{n}}
\end{aligned}
$$

From (4.56) and (4.62), we get

$$
\alpha_{u}=\frac{1}{2 u}(\partial-\bar{\partial}) \frac{\partial}{\partial b}\left\{\operatorname{Tr}_{s}\left[\exp \left(-\mathscr{B}_{u}^{2}+b N_{H}\right)\right]\right\}_{b=0}
$$

From (4.54) and (4.63), we deduce (4.36). 
We now prove (4.37). By proceeding as in (4.38), we get (4.64)

$$
\begin{aligned}
& \frac{\partial}{\partial b} \operatorname{Tr}_{s}\left[\exp \left(-\mathscr{B}_{u}^{2}+b N_{H}\right)\right]_{b=0} \\
& \quad=\int_{0}^{1} d s \int_{M_{R_{x}} \times N_{R_{x}}} \operatorname{Tr}_{s}\left[P_{s}^{x, u}\left(Y, Y^{\prime}\right) N_{H} P_{1-s}^{x, u}\left(Y^{\prime}, Y\right)\right] \frac{d v_{M}\left(Y^{\prime}\right)}{(2 \pi)^{m}} \frac{d v_{N}(Y)}{(2 \pi)^{n}} .
\end{aligned}
$$

If $Y^{\prime} \in M_{R, x}$, set $Y^{\prime}=Y^{\prime L}+Y^{\prime N}$ with $Y^{\prime L} \in L_{R, x}$ and $Y^{\prime N} \in N_{R, x}$. Using equation (4.21) and the fact that supertraces vanish on supercommutators, we find that if $Y \in N_{R, x}$ and $Y^{\prime} \in M_{R, x}$, then

$$
\begin{aligned}
& \operatorname{Tr}_{s}\left[P_{s}^{x, u}\left(Y, Y^{\prime}\right) N_{H} P_{1-s}^{x, u}\left(Y^{\prime}, Y\right)\right] \\
& \quad=\operatorname{Tr}_{s}\left[N_{H} P_{1-s}^{x, u}\left(Y^{\prime N}, Y-Y^{\prime L}\right) P_{s}^{x, u}\left(Y-Y^{\prime L}, Y^{\prime N}\right)\right] .
\end{aligned}
$$

From (4.64) and (4.65), we get

$$
\begin{aligned}
& \frac{\partial}{\partial b}\left\{\operatorname{Tr}_{s}\left[\exp \left(-\mathscr{B}_{u}^{2}+b N_{H}\right)\right]\right\}_{b=0} \\
& \quad=\int_{0}^{1} d s \int_{M_{R_{x}} \times N_{R_{x}}} \operatorname{Tr}_{s}\left[N_{H} P_{s}^{x, u}\left(Y, Y^{\prime}\right) P_{1-s}^{x, u}\left(Y^{\prime}, Y\right)\right] \frac{d v_{M}\left(Y^{\prime}\right)}{(2 \pi)^{m}} \frac{d v_{N}(Y)}{(2 \pi)^{n}} .
\end{aligned}
$$

Also since $P_{s}^{x, u}\left(Y, Y^{\prime}\right)$ is a semigroup, we know that for $0<s \leq 1$ and $Y \in N_{R, x}$

$$
P_{1}^{x, u}(Y, Y)=\int_{0}^{1} d s \int_{M_{R_{x}}} P_{s}^{x, u}\left(Y, Y^{\prime}\right) P_{1-s}^{x, u}\left(Y^{\prime}, Y\right) \frac{d v_{M}\left(Y^{\prime}\right)}{(2 \pi)^{m}} .
$$

(4.37) follows from (4.66) and (4.67).

Remark 4.7. In Theorem 4.6, we can replace $N_{H}$ by $N_{H}+c I \quad(c \in \mathbb{C})$. This can be directly seen in formula (4.36), since the forms $\operatorname{Tr}_{s}\left[\exp \left(-\mathscr{B}_{u}^{2}\right)\right]$ are closed. Also observe that identity (4.37) is trivial for usual (i.e., nongeneralized) supertraces. Finally note that (4.54) and (4.56) also make sense when $B$ is a $C^{\infty}$ noncomplex manifold. In the sequel, we will not keep track of the form $\alpha_{u}$. As will be clear from Theorem 8.5, integration of equation (4.54) in the variable $u$ produces only standard Chern-Simons characteristic classes on $B$. In this respect equation (4.36) is much more interesting.

(f) The asymptotics of the generalized supertrace as $u \rightarrow 0$. Let $\omega_{u}(b)$ be a family of smooth forms on $B$ which depend smoothly on $b \in \mathbb{C}$. We will write that as $u \rightarrow 0, \omega_{u}(b)=\omega_{0}(b)+O(u)$ if for any $k \in \mathbb{N}$, the sup of the norm of the derivatives of order $\leq k$ of $\omega_{u}(b)-\omega_{0}(b)$ on $B \times\{b \in \mathbb{C} ;|b| \leq 1\}$ can be dominated by $C_{k} u$ for $u \leq 1$.

Recall that the Todd polynomial Td is an ad-invariant power series defined on $(p, p)$ complex matrices such that if $C$ has diagonal entries $x_{1}, \ldots, x_{p}$, then

$$
\operatorname{Td}(C)=\prod_{1}^{p} \frac{x_{i}}{1-e^{-x_{i}}}
$$


Let $\left(\mathrm{Td}^{-1}\right)^{\prime}(C)$ be the ad-invariant power series defined by

$$
\left(\mathrm{Td}^{-1}\right)^{\prime}(C)=\frac{\partial}{\partial b}\left[\mathrm{Td}^{-1}(C+b I)\right]_{b=0} .
$$

If $C$ has diagonal entries $x_{1}, \ldots, x_{p}$, then

$$
\left(\mathrm{Td}^{-1}\right)^{\prime}(C)=\frac{\partial}{\partial b}\left[\prod_{1}^{p} \frac{1-e^{-\left(x_{i}+b\right)}}{x_{i}+b}\right]_{b=0} .
$$

The power series $\left(\mathrm{Td}^{-1}\right)^{\prime}$ was introduced in [B5, Definition 4.2].

We study here the asymptotics of the form $\operatorname{Tr}_{s}\left[\exp \left(-\mathscr{B}_{u}^{2}+b N_{H}\right)\right]$ as $u \rightarrow 0$. Note that the result which follows will be reobtained in Remark 7.4. Let $I_{N}$ be the identity on $N$.

Theorem 4.8. For any $b \in \mathbb{C}$, as $u \rightarrow 0$

$$
\operatorname{Tr}_{s}\left[\exp \left(-\mathscr{B}_{u}^{2}+b N_{H}\right)\right]=\frac{\operatorname{Td}\left(-R^{M}\right)}{\operatorname{Td}\left(-R^{N}-b I_{N}\right)}+O(u) .
$$

In particular, as $u \rightarrow 0$

$$
\begin{aligned}
\operatorname{Tr}_{s}\left[\exp \left(-\mathscr{B}_{u}^{2}\right)\right] & =\frac{\operatorname{Td}\left(-R^{M}\right)}{\operatorname{Td}\left(-R^{N}\right)}+O(u), \\
\frac{\partial}{\partial b}\left\{\operatorname{Tr}_{s}\left[\exp \left(-\mathscr{B}_{u}^{2}+b N_{H}\right)\right]\right\}_{b=0} & =-\operatorname{Td}\left(-R^{M}\right)\left(\operatorname{Td}^{-1}\right)^{\prime}\left(-R^{N}\right)+O(u) .
\end{aligned}
$$

Proof. Clearly, by (4.32), we have

$$
\operatorname{Tr}_{s}\left[\exp \left(-\mathscr{B}_{u}^{2}+b N_{H}\right)\right]=\int_{N_{R_{x}}} \frac{\operatorname{Tr}_{s}\left[P_{s}^{x, u, b}(Y / \sqrt{u}, Y \sqrt{u})\right]}{(2 \pi u)^{n}} d v_{N}(Y) .
$$

Recall that $\pi$ is the projection $N \rightarrow B$. From (2.2), we easily deduce that $T^{H} N \cong \pi^{*} T B$ as smooth vector bundles on $N$. From (2.1), we then find that

$$
\Lambda\left(T_{R}^{*} N\right)=\Lambda\left(T_{R}^{*} B\right) \hat{\otimes} \Lambda\left(N_{R}^{*}\right) .
$$

The grading of $\Lambda\left(N_{R}^{*}\right)$ defines a partial grading on $\Lambda\left(T_{R}^{*} N\right)$. If $\omega \in \Lambda\left(T_{R}^{*} N\right)$, we denote by $\omega^{\max }$ the component of $\omega$ which has top partial degree $\operatorname{dim} N_{R}=$ $2 n$. Of course if $\omega$ is a smooth section of $\Lambda\left(T_{R}^{*} N\right)$ with compact support, then

$$
\int_{N_{R}} \omega=\int_{N_{R}} \omega^{\max }
$$

The key step in our proof of Theorem 4.8 is as follows.

Theorem 4.9. For any $Y \in N_{R}$

$$
\begin{aligned}
\lim _{u \downarrow 0} & \frac{1}{(2 \pi u)^{n}} \operatorname{Tr}_{s}\left[P_{1}^{x, u, b}\left(\frac{Y}{\sqrt{u}}, \frac{Y}{\sqrt{u}}\right)\right] d v_{N}(Y) \\
\quad & =\operatorname{Td}\left(-R^{M}\right)\left(\frac{-i}{2 \pi}\right)^{n} \operatorname{Tr}_{s}\left[\exp \left(-\left(\nabla^{N}+\sqrt{-1} \frac{\hat{c}(\mathbf{J} Y)}{\sqrt{2}}\right)^{2}+b N_{H}\right)\right]^{\max } .
\end{aligned}
$$


There exist $c, C>0$, such that for $0<u \leq 1,|b| \leq 1$ and $Y \in N_{R}$

$$
\left(\frac{1}{2 \pi u}\right)^{n}\left|\operatorname{Tr}_{s}\left[P_{1}^{x, u, b}\left(\frac{Y}{\sqrt{u}}, \frac{Y}{\sqrt{u}}\right)\right]\right| \leq c \exp \left(-C|Y|^{2}\right) \text {. }
$$

Proof. We use the notation of the proof of Theorem 4.1. Set $Q=Q_{(0,0)}^{1}$. Let $\stackrel{\circ}{Y}_{t}$ be a process whose probability law is $Q$. By [Si1, p. 41], the law of the process $Y_{t}=Y / \sqrt{u}+\stackrel{\circ}{Y}_{t}$ is exactly $Q_{(Y / \sqrt{u}, Y / \sqrt{u})}^{1}$.

Let $V_{t}$ be the solution of the differential equation

$$
\begin{gathered}
\frac{d V}{d t}=V\left[-\sqrt{u} S-\frac{\sqrt{-1}}{\sqrt{2}} \sqrt{u} \hat{c}\left(\mathbf{J} A P^{L} \stackrel{\circ}{Y}\right)-\frac{1}{2} \operatorname{Tr}\left[R^{M}\right]-\widehat{R^{N}}+b N_{H}\right], \\
V_{0}=I_{\Lambda N_{R, x}^{*}} \otimes_{R} \mathbb{C} .
\end{gathered}
$$

By (4.11), we know that

$$
\begin{aligned}
& \left(\frac{1}{2 \pi u}\right)^{n} \operatorname{Tr}_{s}\left[P_{1}^{x, u, b}\left(\frac{Y}{\sqrt{u}}, \frac{Y}{\sqrt{u}}\right)\right] \\
& =E^{Q}\left[\exp \left\{\frac{1}{2} \int_{0}^{1}\left\langle R^{M} \stackrel{\circ}{Y}, d \stackrel{\circ}{Y}\right\rangle-\frac{1}{2} \int_{0}^{1}\left|Y+\sqrt{u} P^{N} \stackrel{\circ}{Y}_{t}\right|^{2} d t\right\} \frac{\operatorname{Tr}_{s}\left[V_{1}\right]}{(2 \pi u)^{n}}\right] .
\end{aligned}
$$

Now remember that by $\S 1(\mathrm{~b}), \Lambda\left(\bar{N}^{*}\right)$ is a $c\left(N_{R}\right)$ Clifford module. More precisely, let $(\operatorname{det} N)^{1 / 2}$ be a locally defined holomorphic square root of the line bundle $\operatorname{det} N$. Then if

$$
F=\Lambda\left(\bar{N}^{*}\right) \otimes(\operatorname{det} N)^{-1 / 2}
$$

by [H, Theorem 2.2], $F$ is the Hermitian vector bundle of $N_{R}$ spinors. Also if

$$
F_{+}=\Lambda^{\text {even }}\left(\bar{N}^{*}\right) \otimes(\operatorname{det} N)^{-1 / 2}, \quad F_{-}=\Lambda^{\text {odd }}\left(\bar{N}^{*}\right) \otimes(\operatorname{det} N)^{-1 / 2},
$$

then $F=F_{+} \oplus F_{-}$and $F_{+}$and $F_{-}$are the vector bundles of positive and negative $N_{R}$ spinors. Let $e_{1}, \ldots, e_{2 n}$ be an orthonormal oriented base of $N_{R}$. By [AB, p. 484], $c\left(e_{1}\right) \cdots c\left(e_{2 n}\right)$ is the only monomial in $c\left(N_{R}\right)$ whose supertrace evaluated on $F=F_{+} \oplus F_{-}$is nonzero, and moreover

$$
\operatorname{Tr}_{s}\left[c\left(e_{1}\right) \cdots c\left(e_{2 n}\right)\right]=(-2 i)^{n} \text {. }
$$

Recall formula (3.35) for $S$ :

$$
S=\frac{\sqrt{-1}}{2} \sum_{1}^{2 n} c\left(e_{i}\right) \hat{c}\left(\mathbf{J} e_{i}\right)
$$

Note that in equation (4.78) for $V,-\sqrt{u} S$ is the only term where Clifford variables of the type $c\left(e_{i}\right)$ appear (it is very important here to distinguish $c\left(e_{i}\right)$ from $\left.\hat{c}\left(e_{i}\right)\right)$; all other terms act on $\Lambda\left(N^{*}\right)$. Besides, in $-\sqrt{u} S$, the Clifford variables $c\left(e_{i}\right)$ have exactly the weight $\sqrt{u}$.

Let $d y^{1}, \ldots, d y^{2 n}$ be the base of $N_{R}^{*}$ which is dual to $e_{1}, \ldots, e_{2 n}$. If $\eta \in \Lambda\left(N_{R}^{*}\right)$, we define $\eta_{0} \in \mathbb{C}$ by

$$
\eta_{0} d y^{1} \wedge \cdots \wedge d y^{2 n}=\eta^{\max } .
$$


By using Duhamel's formula, we can then expand $V_{1}$ into powers of $\sqrt{u}$. By (4.80), and by proceeding as in [B1, Theorem 4.12], we find easily that as $u \rightarrow 0$ (4.81)

$$
\begin{aligned}
\frac{\operatorname{Tr}_{s}\left[V_{1}\right]}{u^{n}} \rightarrow & (-2 i)^{n}\left\{\operatorname{Tr}_{s}^{\Lambda N^{*}}\left[\exp \left(-\frac{\sqrt{-1}}{2} \sum_{1}^{2 n} d y^{i} \hat{c}\left(\mathbf{J} e_{i}\right)-\widehat{R^{N}}+b N_{H}\right)\right]\right\}_{0} \\
& \times \exp \left(-\frac{1}{2} \operatorname{Tr}\left[R^{M}\right]\right) .
\end{aligned}
$$

The same argument shows that there exist $c, C>0$ such that for $0<u \leq 1$

$$
\left|\frac{\operatorname{Tr}_{s}\left[V_{1}\right]}{u^{n}}\right| \leq c \exp \left\{C \int_{0}^{1}\left|P_{L} \stackrel{\circ}{Y}_{t}\right| d t\right\}
$$

(we can in fact replace (4.82) by a sharper inequality since $A P^{L} \stackrel{\circ}{Y}$ contains Grassmann variables in $\Lambda\left(T_{R}^{*} B\right)$ ).

The bounds (4.17), (4.18), and (4.82) show that we can now use dominated convergence in the expectation in the right-hand side of (4.79). We find from (4.79) and (4.81) that

$$
\begin{aligned}
\lim _{u \downarrow 0} & \frac{1}{(2 \pi u)^{n}} \operatorname{Tr}_{s}\left[P_{1}^{x, u, b}\left(\frac{Y}{\sqrt{u}}, \frac{Y}{\sqrt{u}}\right)\right] d v_{M}(Y) \\
= & E^{Q}\left[\left\{\exp \frac{1}{2} \int_{0}^{1}\left\langle R^{M} \stackrel{\circ}{Y}, d \stackrel{\circ}{Y}\right\rangle-\frac{1}{2} \operatorname{Tr}\left[R^{M}\right]\right\}\right] \\
& \times\left(\frac{-2 i}{2 \pi}\right)^{n} \operatorname{Tr}_{s}^{\Lambda N^{*}}\left[\exp \left(-\widehat{R^{N}}-\frac{\sqrt{-1}}{2} \sum_{1}^{2 n} d y^{i} \hat{c}\left(\mathbf{J} e_{i}\right)-\frac{|Y|^{2}}{2}+b N_{H}\right)\right]^{\max } .
\end{aligned}
$$

Let $\widehat{A}$ be the complex Hirzebruch polynomial. Namely, $\widehat{A}$ is the ad-invariant polynomial which is such that if the $(n, n)$ matrix $C$ has diagonal elements $x_{1}, \ldots, x_{n}$, then

$$
\widehat{A}(C)=\prod_{1}^{n} \frac{x_{i} / 2}{\sinh \left(x_{i} / 2\right)}
$$

Then by a formula of P. Lévy [Le; B8, Theorem 3.17], we know that

Therefore,

$$
E^{Q}\left[\exp \left\{\frac{1}{2} \int_{0}^{1}\left\langle R^{M} \stackrel{\circ}{Y}, d \stackrel{\circ}{Y}\right\rangle\right\}\right]=\widehat{A}\left(R^{M}\right) .
$$

$$
E^{Q}\left[\exp \left\{\frac{1}{2}\left\langle R^{M} \stackrel{\circ}{Y}, d \stackrel{\circ}{Y}\right\rangle-\frac{1}{2} \operatorname{Tr}\left[R^{M}\right]\right\}\right]=\operatorname{Td}\left(-R^{M}\right) .
$$

Also it is clear that

$$
\begin{aligned}
& \left(\frac{-2 i}{2 \pi}\right)^{n} \operatorname{Tr}_{s}^{\Lambda N^{*}}\left[\exp \left(-\widehat{R^{N}}-\frac{\sqrt{-1}}{2} \sum_{1}^{2 n} d y^{i} \hat{c}\left(\mathbf{J} e_{i}\right)-\frac{|Y|^{2}}{2}+b N_{H}\right)\right]^{\max } \\
& =\left(\frac{-i}{2 \pi}\right)^{n} \operatorname{Tr}_{s}^{\Lambda N^{*}}\left[\exp \left(-\widehat{R^{N}}-\frac{\sqrt{-1}}{2} \sum_{1}^{2 n} d y \frac{\hat{c}\left(\mathbf{J} e_{i}\right)}{\sqrt{2}}-\frac{|Y|^{2}}{2}+b N_{H}\right)\right]^{\max } .
\end{aligned}
$$


On the other hand, since $\left(\nabla^{N}\right)^{2}=\widehat{R^{N}}$, we have

$$
\left(\nabla^{N}+\sqrt{-1} \frac{\hat{c}(\mathbf{J} Y)}{\sqrt{2}}\right)^{2}=\widehat{R^{N}}+\sqrt{-1} \frac{\nabla^{N} \hat{c}(\mathbf{J} Y)}{\sqrt{2}}+\frac{|Y|^{2}}{2} .
$$

Now it is clear that

$$
\nabla^{N} \hat{c}(\mathbf{J} Y)=\sum_{1}^{2 n} d y^{i} \hat{c}\left(\mathbf{J} e_{i}\right)
$$

From (4.83)-(4.88), we get (4.76).

We now prove (4.77). Using (4.79) and (4.82), we obtain (4.89)

$$
\begin{aligned}
&\left(\frac{1}{2 \pi u}\right)^{n} \mid \operatorname{Tr}_{s}\left[P_{1}^{x, u, b}\left(\frac{Y}{\sqrt{u}}, \frac{Y}{\sqrt{u}}\right)\right] \mid \\
& \leq E^{Q}\left[\left\|\exp \left\{\frac{1}{2} \int_{0}^{1}\left\langle R^{M} \stackrel{\circ}{Y}, d \stackrel{\circ}{Y}\right\rangle\right\}\right\|\right. \\
&\left.\quad \times \exp \left\{-\frac{1}{2} \int_{0}^{1}\left|Y+\sqrt{u} P^{N} \stackrel{\circ}{Y}_{t}\right|^{2} d t\right\} \exp \left\{C \int_{0}^{1}\left|P^{L} Y_{t}\right| d t\right\}\right] .
\end{aligned}
$$

By Hölder's inequality, (4.17), and (4.18), we find that

$$
\begin{aligned}
& \left(\frac{1}{2 \pi u}\right)^{n}\left|\operatorname{Tr}_{s}\left[P_{1}^{x, u, b}\left(\frac{Y}{\sqrt{u}}, \frac{Y}{\sqrt{u}}\right)\right]\right| \\
& \quad \leq c\left[E^{Q}\left(\exp \left\{-\frac{3}{2} \int_{0}^{1}\left|Y+\sqrt{u} P^{N} \stackrel{Y}{t}_{t}\right|^{2} d t\right\}\right)\right]^{1 / 3}\left(1+|Y|^{q}\right) .
\end{aligned}
$$

Using (4.19), we get

$$
\begin{aligned}
E^{Q}[ & \left.\exp \left\{-\frac{3}{2} \int_{0}^{1}\left|Y+\sqrt{u} P^{N} Y_{t}^{0}\right|^{2} d t\right\}\right] \\
& =E^{Q_{(Y / \sqrt{u}, Y / \sqrt{u})}}\left[\exp \left\{-\frac{3}{2} u \int_{0}^{1}\left|P^{N} Y_{t}\right|^{2} d t\right\}\right] \\
& =\left(\frac{(3 u)^{1 / 2}}{\sinh \left((3 u)^{1 / 2}\right)}\right)^{n} \exp \left\{\frac{-(3 u)^{1 / 2}}{\sinh \left((3 u)^{1 / 2}\right)} \frac{\left(\cosh \left((3 u)^{1 / 2}\right)-1\right)}{u}|Y|^{2}\right\} .
\end{aligned}
$$

From (4.90) and (4.91), it is now clear that (4.77) holds.

Proof of Theorem 4.8 (continued). By using formula (4.23) in [B5], derived from Mathai-Quillen [MQ, Theorem 4.5], we know that

$$
\left(\frac{-i}{2 \pi}\right)^{n} \int_{N} \operatorname{Tr}_{s}\left[\exp \left(-\left(\nabla^{N}+\frac{\sqrt{-1} \hat{c}(\mathbf{J} Y)}{\sqrt{2}}\right)^{2}+b N_{H}\right)\right]=\operatorname{Td}^{-1}\left(-R^{N}-b I_{N}\right) \text {. }
$$

Using Theorem 4.9 and (4.92), we get

$$
\lim _{u \downarrow 0} \operatorname{Tr}_{s}\left[\exp \left(-\mathscr{B}_{u}^{2}+b N_{H}\right)\right]=\frac{\operatorname{Td}\left(-R^{M}\right)}{\operatorname{Td}\left(-R^{N}-b I_{N}\right)} .
$$


The proof that the sup of the norm of the derivatives of order $\leq k$ of the form

$$
\operatorname{Tr}_{s}\left[\exp \left(-\mathscr{B}_{u}^{2}+b N_{H}\right)\right]-\frac{\operatorname{Td}\left(-R^{M}\right)}{\operatorname{Td}\left(-R^{N}-b I_{N}\right)}
$$

can be dominated by $\mathrm{Cu}$ as $u \rightarrow 0$ requires more work which can be easily done. In particular, instead of (4.83), we now must obtain an asymptotic expansion of the left-hand side of (4.83), by using essentially the same techniques as before. This is left to the reader. So (4.71) is proved. By differentiating with respect to $b$ at $b=0$ (this is possible by using, say, analyticity in the variable $b$ ), we obtain the second line in (4.72). The proof of Theorem 4.8 is complete.

Remark 4.10. Although the cancellations which appear in the proof of Theorem 4.9 are closely related to similar cancellations which appear in the proof of the local index theorem $[\mathrm{BeV}, \mathrm{B} 8, \mathrm{Ge}]$, the situation considered here is essentially different from [BeV, B8, Ge]. In particular, the fact that the total space of $N$ scales adequately plays an essential role in the proof of (4.71).

Remark 4.11. Let $\Phi$ be the linear map from $\Lambda^{\text {even }}\left(T_{R}^{*} B\right)$ into itself which sends $\alpha \in \Lambda^{2 p}\left(T_{R}^{*} B\right)$ into $(2 \pi i)^{-p} \alpha$. It is tempting to consider the forms $\Phi\left(\operatorname{Tr}_{s}\left[\exp \left(-\mathscr{B}_{u}^{2}\right)\right]\right)$ as generalized Chern character forms $\operatorname{ch}\left(D^{M}+\sqrt{u} V\left(P^{N} Y\right)\right)$ of the family $D^{M}+\sqrt{u} V\left(P^{N} Y\right)$. In fact they are obtained as generalized supertraces on an adequate von Neumann algebra. The construction of these forms generalizes the constructions of [B1].

If $N=\{0\}$, then $\mathscr{B}_{u}$ does not depend on $u$. From (4.60), we find that if $N=\{0\}$, then

$$
\Phi\left(\operatorname{Tr}_{s}\left[\exp \left(-\mathscr{B}_{u}^{2}\right)\right]\right)=\operatorname{Td}\left(\frac{-R^{L}}{2 \pi}\right) .
$$

Equivalently, if $N=\{0\}, \Phi\left(\operatorname{Tr}_{s}\left[\exp \left(-\mathscr{B}_{u}^{2}\right)\right]\right)=\operatorname{ch}\left(D^{L}\right)$, and so

$$
\operatorname{ch}\left(D^{L}\right)=\operatorname{Td}\left(\frac{-R^{L}}{2 \pi}\right) \text {. }
$$

If $\xi$ is a holomorphic vector bundle on $B$, the Atiyah-Singer-Riemann-RochHirzebruch formula for the Euler characteristic $\chi(\xi)$ of $\xi$ can then be written in the form

$$
\chi(\xi)=\int_{B} \operatorname{ch}\left(D^{T B}\right) \operatorname{ch}(\xi) .
$$

Of course the computations in the proof of Theorem 4.9 are intimately related with the final stage of the probabilistic proof of the Atiyah-Singer Index Theorem in [B1, B8]. For essentially the same reasons, the Getzler operator (3.19) (with $M=T B$ ) also appears at the final stage of the proof of the Index Theorem in Getzler [Ge].

Formulas (4.95)-(4.97) may be the starting point of a Chern-Weil theory in $K$-homology. 
(g) A modified version of the superconnection $\mathscr{B}_{u}$. Here we introduce a new superconnection $\mathscr{C}_{u}$, which is essentially equivalent to the superconnection $\mathscr{B}_{u}$, and whose curvature $\mathscr{C}_{u}^{2}$ is also very simple. Although $\mathscr{C}_{u}$ will not reappear in the sequel, some readers may find it more pleasant to use in the constructions which will follow. (The operator $\mathscr{C}_{u}^{2}$ plays an important role in another proof of Theorem 7.7 given in [BL2, §14].)

If $Y \in M_{R}$, we define $c\left(A P^{L} Y\right)$ as in (4.23). Let $e_{1}, \ldots, e_{2 m}$ be an orthonormal base of $M_{R}$.

Theorem 4.12. Let $\mathscr{C}_{u}$ be the superconnection

$$
\mathscr{C}_{u}=\exp \left(c \frac{\left(A P^{L} Y\right)}{\sqrt{2}}\right) \mathscr{B}_{u} \exp \left(-c \frac{\left(A P^{L} Y\right)}{\sqrt{2}}\right)
$$

Then

$$
\begin{aligned}
\mathscr{C}_{u}^{2}= & -\frac{1}{2} \sum_{1}^{2 m}\left(\nabla_{e_{i}}+\frac{1}{2}\left\langle\left(R^{M}-P^{L} A^{2} P^{L}\right) Y, e_{i}\right\rangle-\frac{c\left(A P^{L} e_{i}\right)}{\sqrt{2}}\right)^{2} \\
& +\frac{u\left|P^{N} Y\right|^{2}}{2}+\sqrt{u} S+\frac{1}{2} \operatorname{Tr}\left[R^{M}\right]+\widehat{R^{N}}
\end{aligned}
$$

Proof. It is clear that $\mathscr{C}_{u}$ is a superconnection. Moreover,

$$
\mathscr{C}_{u}^{2}=\exp \left(c \frac{\left(A P^{L} Y\right)}{\sqrt{2}}\right) \mathscr{B}_{u}^{2} \exp \left(-c \frac{\left(A P^{L} Y\right)}{\sqrt{2}}\right) .
$$

Now by proceeding as in (4.24)-(4.27), we find that

$$
\exp \left(c \frac{\left(A P^{L} Y\right)}{\sqrt{2}}\right)\left(S+\frac{\sqrt{-1}}{\sqrt{2}} \hat{c}\left(\mathbf{J} A P^{L} Y\right)\right) \exp \left(-c \frac{\left(A P_{L} Y\right)}{\sqrt{2}}\right)=S \text {. }
$$

Also

$$
\begin{gathered}
{\left[c\left(A P^{L} Y\right), \nabla_{e_{i}}\right]=-c\left(A P^{L} e_{i}\right),} \\
{\left[c\left(A P^{L} Y\right),\left[c\left(A P^{L} Y\right), \nabla_{e_{i}}\right]=-\left[c\left(A P^{L} Y\right), c\left(A P^{L} e_{i}\right)\right]\right.} \\
=-2\left\langle A^{2} P^{L} Y, P^{L} e_{i}\right\rangle, \\
{\left[c\left(A P^{L} Y\right),\left[c\left(A P^{L} Y\right),\left[c\left(A P^{L} Y\right), \nabla_{e_{i}}\right]\right]\right]=0 .}
\end{gathered}
$$

From (4.102), we get

$$
\begin{aligned}
& \exp \left(c \frac{\left(A P^{L} Y\right)}{\sqrt{2}}\right) \nabla_{e_{i}} \exp \left(-c \frac{\left(A P_{L} Y\right)}{\sqrt{2}}\right) \\
& =\nabla_{e_{i}}-c \frac{\left(A P^{L} e_{i}\right)}{\sqrt{2}}-\frac{1}{2}\left\langle A^{2} P^{L} Y, P^{L} e_{i}\right\rangle .
\end{aligned}
$$

Using (4.101)-(4.103) and formula (3.36) for $\mathscr{B}_{u}^{2}$, we obtain (4.99). 


\section{AN EXTENSION OF A FORMULA OF MATHAI-QUILlEN: THE TIME-DEPENDENT CASE}

In [MQ], Mathai and Quillen used superconnections to construct Gaussian shaped Thom forms on finite-dimensional Euclidean vector bundles. As will be clear in $\S 9$, part of our work is related to the constructions of equivariant Thom forms on certain infinite-dimensional equivariant vector bundles.

In particular, in our computation of $\S 7$ of the forms $\operatorname{Tr}_{s}\left[\exp \left(-s \mathscr{B}_{u}^{2}+b N_{H}\right)\right]$, we need a time-dependent version of a formula of Mathai-Quillen [MQ, (2.13)] for the supertrace of $\exp (\alpha+\beta)$, where $\alpha$ is of length two in the Clifford algebra $c(E)$ of an even-dimensional Euclidean vector space $E$, and $\beta \in \Lambda^{1}\left(F^{*}\right) \hat{\otimes} c^{1}(E)$ (where $F$ is a finite-dimensional vector space). Here we extend their formula to the case where $\beta$ is time dependent, and where $\exp (\alpha+\beta)$ is replaced by a path-ordered exponential.

This section is self-contained.

In fact, let $E$ be a real oriented Euclidean vector space of even dimension $2 p$. Let $e_{1}, \ldots, e_{2 p}$ be an orthonormal oriented base of $E$. Let $c(E)$ be the Clifford algebra of $E$. Then $c(E)$ is naturally $\mathbb{Z}$ graded. If $X \in E$, we note by $c(X)$ the corresponding element in $c(E)$. Set

$$
\tau=i^{p} c\left(e_{1}\right) \cdots c\left(e_{2 p}\right) \text {. }
$$

Then $\tau^{2}=1$. Let $S$ be the Hermitian vector space of $E$ spinors. Then $c(E)$ acts on $S$. Set

$$
S_{ \pm}=(s \in S ; \tau s= \pm s) .
$$

Then $S=S_{+} \oplus S_{-}$and $S_{+}$and $S_{-}$are the vector spaces of positive and negative spinors.

Let $\mathscr{G}$ be the Lie algebra of real antisymmetric endomorphisms of $E$. If $B \in \mathscr{G} \otimes_{R} \mathbb{C}$, let $c(B) \in c^{2}(E)$ be given by

$$
c(B)=\sum \frac{1}{4}\left\langle B e_{i}, e_{i}\right\rangle c\left(e_{i}\right) c\left(e_{j}\right) .
$$

Then $B \in \mathscr{G} \otimes_{R} \mathbb{C} \rightarrow c(B) \in c^{2}(E)$ is a Lie algebra homomorphism [ABS].

$\operatorname{End}(S)$ is a $\mathbb{Z}_{2}$-graded algebra, with the even (resp. odd) elements of $\operatorname{End}(S)$ commuting (resp. anticommuting) with $\tau$. Also

$$
\operatorname{End}(S) \cong c(E), \quad \operatorname{End}^{\text {even }}(S) \cong c^{\text {even }}(E), \quad \operatorname{End}^{\text {odd }}(S) \cong c^{\text {odd }}(E) .
$$

Let $F$ be a finite-dimensional real vector space. The algebra $\Lambda\left(F^{*}\right)$ is naturally $\mathbb{Z}_{2}$ graded. Our computations will now be done in the algebra

$$
\Lambda\left(F^{*}\right) \hat{\otimes} \text { End } S \cong \Lambda\left(F^{*}\right) \hat{\otimes} c(E) .
$$

End $S$ is naturally equipped with a supertrace $\operatorname{Tr}_{s}=\operatorname{Tr}[\tau \cdot]$. As in Quillen [Q1], we extend $\operatorname{Tr}_{s}$ into a linear map from $\Lambda\left(F^{*}\right) \widehat{\otimes}$ End $S$ into $\Lambda\left(F^{*}\right)$, with the convention that if $\omega \in \Lambda\left(F^{*}\right)$ and $A \in$ End $S$, then

$$
\operatorname{Tr}_{s}[\omega A]=\omega \operatorname{Tr}_{s}[A] \text {. }
$$


Let $\theta_{.}: t \in[0,1] \rightarrow \theta_{t} \in \Lambda^{1}\left(F^{*}\right) \otimes E$ be a smooth map. Then if $f^{1}, \ldots$, $f^{\operatorname{dim} F}$ is a base of $F^{*}$, we can write $\theta_{t}$ in the form

$$
\theta_{t}=\sum_{1}^{\operatorname{dim} F} f^{\alpha} \theta_{\alpha, t}
$$

with $\theta_{t}^{\alpha} \in E$ and $1 \leq \alpha \leq \operatorname{dim} F$. Set

$$
c\left(\theta_{t}\right)=-\sum_{1}^{\operatorname{dim} F} f^{\alpha} c\left(\theta_{\alpha, t}\right) \in\left(\Lambda\left(F^{*}\right) \hat{\otimes} c(E)\right)^{\text {even }} .
$$

Note that our sign conventions in (5.3) are the same as in (3.34).

Now take $B \in \mathscr{G}$. Consider the differential equation

$$
\frac{d G}{d t}=G\left(c(B)+c\left(\theta_{t}\right)\right), \quad G(0)=I_{S} .
$$

In (5.4), $G_{t}$ lies in $\left(\Lambda\left(F^{*}\right) \hat{\otimes} c(E)\right)^{\text {even }}$.

We will now give a formula for $\operatorname{Tr}_{s}\left[G_{1}\right]$. In the case where $\theta_{t}$ is constant, i.e., does not depend on $t$, such a formula was already given in Mathai-Quillen [MQ, (2.13)]. In fact if $\theta_{t}=\theta$, then

$$
\operatorname{Tr}_{s}[\exp (c(B)+c(\theta))]=\operatorname{Tr}_{s}[\exp (c(B))] \exp \left\{\left\langle B^{-1} \theta, \theta\right\rangle\right\} .
$$

To make sense of Mathai-Quillen's formula (5.5), we first assume that $B$ is invertible. Then $\left\langle B^{-1} \theta, \theta\right\rangle \in \Lambda^{2}\left(F^{*}\right)$. If $B$ has diagonal blocks

$$
\left[\begin{array}{cc}
0 & -\beta_{j} \\
\beta_{j} & 0
\end{array}\right] \quad(1 \leq j \leq p)
$$

(here $B$ is calculated on an oriented orthonormal base), then by [B8, Proposition 1.2]

$$
\operatorname{Tr}_{s}[\exp c(B)]=\prod_{1}^{p}\left(e^{-i \beta_{j} / 2}-e^{i \beta_{j} / 2}\right) .
$$

By expanding $\exp \left\{\left\langle B^{-1} \theta, \theta\right\rangle\right\}$ as a finite power series in $\Lambda\left(F^{*}\right)$, one readily verifies that the right-hand side of (5.5) extends by continuity to the case where $B$ is noninvertible.

We now extend Mathai-Quillen's formula to the case of a general timedependent $\theta_{t}$.

In the sequel, we will construct an inverse-when it exists-of the differential operator $\frac{d}{d t}+B$ on $[0,1]$ with periodic boundary conditions. If $t \in \mathbb{R} / \mathbb{Z} \rightarrow$ $f_{t} \in E$ is a smooth periodic function, we consider the differential equation

$$
\frac{d g}{d t}+B g_{t}=f_{t}, \quad g(0)=g(1) .
$$

One readily verifies that if $e^{B}$ has no eigenvalue equal to 1 , then the unique solution $g_{t}$ of (5.7) is given by the formula

$$
g_{t}=e^{-t B}\left\{\int_{0}^{t} e^{s B} f_{s} d s+\left(e^{B}-1\right)^{-1} \int_{0}^{1} e^{s B} f_{s} d s\right\} .
$$


We can also use Fourier series to calculate $g_{t}$. In fact if $f_{t}$ has the Fourier series expansion

$$
f_{t}=\sum f_{n} \exp (2 \pi i n t)
$$

and if $e^{B}$ has no eigenvalue equal to 1 , then $g_{t}$ is given by

$$
g_{t}=\sum\left(2 \pi i n I_{E}+B\right)^{-1} f_{n} e^{2 \pi i n t} .
$$

We now replace $f_{t}$ by $\theta_{t}$, so that

$$
\theta_{t}=\sum \theta_{n} e^{2 \pi i n t} .
$$

Here for $n \in \mathbb{Z}, \theta_{t} \in \Lambda^{1}\left(F^{*}\right) \otimes E$. Then if $e^{B}$ has no eigenvalue equal to 1 ,

$$
\left(\frac{d}{d t}+B\right)^{-1} \theta_{t}=\sum\left(2 \pi i n I_{E}+B\right)^{-1} \theta_{n} e^{2 \pi i n t}
$$

and so

$$
\left\langle\left(\frac{d}{d t}+B\right)^{-1} \theta, \theta\right\rangle=\sum_{n \in \mathbb{Z}}\left\langle\left(2 \pi i n I_{E}+B\right)^{-1} \theta_{n}, \theta_{-n}\right\rangle \in \Lambda^{\mathrm{even}}\left(F^{*}\right) .
$$

The series in (5.13) is clearly convergent.

Therefore,

$$
\exp \left\{\left\langle\left(\frac{d}{d t}+B\right)^{-1} \theta, \theta\right\rangle\right\}=\prod_{n \in \mathbb{Z}} \exp \left\langle\left(2 \pi i n I_{E}+B\right)^{-1} \theta_{n}, \theta_{-n}\right\rangle .
$$

Now when expanding each individual exponential

$$
\exp \left\langle\left(2 \pi i n I_{E}+B\right)^{-1} \theta_{n}, \theta_{-n}\right\rangle
$$

in $\Lambda^{\text {even }}\left(F^{*}\right)$, it is clear that for any $n \in \mathbb{N}$, the expansion stops after at most $\operatorname{dim}(F)$ terms. Also observe that

$$
\sin \left(\frac{\beta}{2}\right)=\frac{\beta}{2} \prod_{k=1}^{+\infty}\left(1-\frac{\beta^{2}}{4 k^{2} \pi^{2}}\right) .
$$

From (5.6) and (5.15), we find that

$$
\operatorname{Tr}_{s}[\exp (c(B))]=(-i)^{p} \prod_{j=1}^{p}\left(\beta_{j} \prod_{k=1}^{+\infty}\left(1-\frac{\beta_{j}^{2}}{4 k^{2} \pi^{2}}\right)\right) .
$$

Using (5.8), (5.10), and (5.16), it is now clear that the expression

$$
\operatorname{Tr}_{s}[\exp (c(B))] \exp \left\{\left\langle\left(\frac{d}{d t}+B\right)^{-1} \theta, \theta\right\rangle\right\},
$$

which is well defined when $\exp (B)$ has no eigenvalue equal to 1 , can be extended by continuity to arbitrary $B$. 
Theorem 5.1. Let $G_{t} \in\left(\Lambda\left(F^{*}\right) \hat{\otimes} c(E)\right)^{\text {even }}$ be given by the differential equation (5.4). Then

$$
\operatorname{Tr}_{s}\left[G_{1}\right]=\operatorname{Tr}_{s}[\exp (c(B))] \exp \left\{\left\langle\left(\frac{d}{d t}+B\right)^{-1} \theta, \theta\right\rangle\right\} .
$$

Proof. We first assume that $e^{B}$ has no eigenvalue equal to 1 . Let $R_{t}$ be the unique solution of the differential equation

$$
\frac{d R}{d t}+B R=-\theta, \quad R(0)=R(1)
$$

Clearly by (5.8), $R_{t} \in \Lambda^{1}\left(F^{*}\right) \otimes E$. Set

$$
C_{t}=-\left\langle B R_{t}, R_{t}\right\rangle \in \Lambda^{2}\left(F^{*}\right) .
$$

Let $H_{t}$ and $V_{t}$ be the solutions of the differential equations

$$
\begin{aligned}
& \frac{d H}{d t}=H\left(-c\left(\frac{d R}{d t}\right)\right), \quad H_{0}=\exp \left(-c\left(R_{0}\right)\right) ; \\
& \frac{d V}{d t}=V\left(c(B)+C_{t}\right), \quad V_{0}=I_{S} .
\end{aligned}
$$

Notice the obvious formula for $V_{t}$ :

$$
V_{t}=\exp \left(t c(B)+\int_{0}^{t} C_{s} d s\right) .
$$

Now $c(d R / d t) \in \Lambda^{1}\left(F^{*}\right) \widehat{\otimes} c^{1}(E)$. Also if $\alpha, \beta \in \Lambda^{1}\left(F^{*}\right) \hat{\otimes} c^{1}(E)$, then $[\alpha, \beta] \epsilon$ $\Lambda^{2}\left(F^{*}\right)$. Therefore, as explained in [B1, Theorem 1.3], $\left(\Lambda^{1}\left(F^{*}\right) \hat{\otimes} c^{1}(E)\right) \oplus$ $\Lambda^{2}\left(F^{*}\right)$ is a Heisenberg algebra, whose center contains $\Lambda^{2}\left(F^{*}\right)$. A trivial application of Campbell-Hausdorff's formula as in [B1, equation (4.31)] shows that

$$
H_{t}=\exp \left\{-c\left(R_{t}\right)+\frac{1}{2} \int_{0}^{t}\left[c(R), c\left(\frac{d R}{d s}\right)\right] d s\right\}
$$

Clearly,

$$
\begin{gathered}
\frac{d}{d t}\left(V_{t} H_{t}\right)=V_{t} H_{t}\left\{H_{t}^{-1}(c(B)+C) H_{t}-c\left(\frac{d R}{d t}\right)\right\}, \\
V_{0} H_{0}=\exp \left(-c\left(R_{0}\right)\right) .
\end{gathered}
$$

Now since $\frac{1}{2} \int_{0}^{t}\left[c\left(R_{s}\right), c(d R / d s)\right] d s \in \Lambda^{2}\left(F^{*}\right)$ and $C \in \Lambda^{2}\left(F^{*}\right)$, we have

$$
H_{t}^{-1}(c(B)+C) H_{t}=\exp \left(c\left(R_{t}\right)\right) c(B) \exp \left(-c\left(R_{t}\right)\right)+C .
$$

Note that $c\left(R_{t}\right) \in \Lambda^{1}\left(F^{*}\right) \hat{\otimes} c^{1}(E)$. Also if $X \in E$

$$
\left[c\left(R_{t}\right), c(X)\right]=2\left\langle R_{t}, X\right\rangle .
$$

Since $2\left\langle R_{t}, X\right\rangle \in \Lambda^{1}\left(F^{*}\right)$, then

$$
\left[c\left(R_{t}\right),\left[c\left(R_{t}\right), c(X)\right]\right]=0 .
$$


From (5.26) and (5.27) we deduce that

$$
\exp \left(c\left(R_{t}\right)\right) c(X) \exp \left(-c\left(R_{t}\right)\right)=c(X)+2\left\langle R_{t}, X\right\rangle .
$$

From (5.28), we get

$$
\exp \left(c\left(R_{t}\right)\right) c(B) \exp \left(-c\left(R_{t}\right)\right)=c(B)-c\left(B R_{t}\right)+\left\langle B R_{t}, R_{t}\right\rangle .
$$

Using (5.25) and (5.29), we deduce that

$$
H_{t}^{-1}(c(B)+C) H_{t}=c(B)-c\left(B R_{t}\right) .
$$

From (5.24) and (5.30), we get

$$
\begin{gathered}
\frac{d\left(V_{t} H_{t}\right)}{d t}=V_{t} H_{t}\left(c(B)-c\left(\frac{d R}{d t}+B R\right)\right), \\
V_{0} H_{0}=\exp \left(-c\left(R_{0}\right)\right) .
\end{gathered}
$$

Using (5.19) and (5.31), we find that

$$
\begin{gathered}
\frac{d\left(V_{t} H_{t}\right)}{d t}=V_{t} H_{t}(c(B)+c(\theta)), \\
V_{0} H_{0}=\exp \left(-c\left(R_{0}\right)\right) .
\end{gathered}
$$

By comparing with (5.4), we obtain

$$
G_{t}=\exp \left(c\left(R_{0}\right)\right) V_{t} H_{t} .
$$

Using (5.22), (5.23), and (5.33) we get

$$
\begin{aligned}
G_{1}= & \exp \left(c\left(R_{0}\right)\right) \exp \left(c(B)+\int_{0}^{1} C_{s} d s\right) \\
& \times \exp \left(-c\left(R_{0}\right)+\frac{1}{2} \int_{0}^{1}\left[c(R), c\left(\frac{d R}{d s}\right)\right] d s\right) .
\end{aligned}
$$

Now

$$
\left[c(R), c\left(\frac{d R}{d s}\right)\right]=2\left\langle R, \frac{d R}{d s}\right\rangle=-2 C-2\langle R, \theta\rangle \in \Lambda^{2}\left(F^{*}\right) .
$$

Using (5.34) and (5.35), we get

$$
G_{1}=\exp \left(c\left(R_{0}\right)\right) \exp (c(B)) \exp \left(-c\left(R_{0}\right)\right) \exp \left(-\int_{0}^{1}\langle R, \theta\rangle d t\right) .
$$

Since, by [Q1], supertraces vanish on supercommutators, from (5.36) we get

$$
\operatorname{Tr}_{s}\left[G_{1}\right]=\operatorname{Tr}_{s}[\exp (c(B))] \exp \left(-\int_{0}^{1}\langle R, \theta\rangle d t\right) .
$$

Of course

$$
-\int_{0}^{1}\langle R, \theta\rangle d t=\left\langle\left(\frac{d}{d t}+B\right)^{-1} \theta, \theta\right\rangle .
$$

(5.18) follows from (5.37) and (5.38). 
If certain eigenvalues of $e^{B}$ are equal to 1 , we can approximate $B$ by elements $B^{\prime}$ in $\mathscr{G}$ such that $e^{B^{\prime}}$ has no eigenvalue equal to 1 . The constructions which were done before Theorem 5.1 show that we can take the obvious limit in formula (5.18) as $B^{\prime}$ tends to $B$. Our theorem is proved.

Remark 5.2. Theorem 5.1 can also be viewed formally as the consequence of a completion of squares in a fermionic integral.

\section{A ONE-PARAMETER DEFORMATION OF THE $\hat{A}$ HIRZEBRUCH POLYNOMIAL}

In this section, we prove various properties of the function $\varphi(u, x)$ which was described in the Introduction. In particular, we show that

- $\varphi(0, x)$ is exactly the inverse of the Hirzebruch polynomial $\widehat{A}(x)$.

- $\varphi(u, x)$ can be simply expressed as an infinite product, or more precisely as the determinant of a differential operator on $S_{1}$.

- $\varphi(u, x)$ appears naturally in the explicit computations of certain supertraces on Clifford algebras, and also in the evaluation of the trace of a harmonic oscillator.

As explained in the Introduction, the function $\varphi(u, x)$ plays a key role in the formulation of our final result.

This section is organized as follows. In (a), we introduce the function $\varphi(u, x)$. In (b), we construct the corresponding multiplicative genus. In (c), we show that $\varphi(u, x)$ appears explicitly in the evaluation of certain finitedimensional supertraces. In (d), we obtain a similar result for the trace of a harmonic oscillator with magnetic field. In Remark 6.7 we give a group theoretic interpretation of the fact that two different computations give similar results. This uses our results of $\S 1$, and also our approach [B11] to the infinitesimal Lefchetz formulas.

Except for notation from $\S 1$, this section is self-contained.

(a) The function $\varphi(u, x)$. If $z \in \mathbb{C}, \sqrt{z}$ denotes an arbitrary (but fixed) square root of $z$. In the sequel, the expressions which we consider do not depend on the choice of the square root.

Definition 6.1. For $u>0$ and $x \in \mathbb{C}$, set

$$
\varphi(u, x)=\frac{4}{u} \sinh \left(\frac{x+\sqrt{x^{2}+4 u}}{4}\right) \sinh \left(\frac{-x+\sqrt{x^{2}+4 u}}{4}\right) .
$$

$x \in \mathbb{C} \rightarrow \varphi(u, x)$ is an even analytic function of $x$, which we can also write in the form

$$
\begin{aligned}
\varphi(u, x)= & \frac{1}{u}\left(e^{\left(x+\sqrt{x^{2}+4 u}\right) / 4}-e^{\left(-x-\sqrt{x^{2}+4 u}\right) / 4}\right) \\
& \times\left(e^{\left(-x+\sqrt{\left.x^{2}+4 u\right)} / 4\right.}-e^{\left(x-\sqrt{\left.x^{2}+4 u\right)} / 4\right.}\right) \\
\varphi(u, x)= & \frac{2}{u}\left(\cosh \left(\frac{\sqrt{x^{2}+4 u}}{2}\right)-\cosh \left(\frac{x}{2}\right)\right) .
\end{aligned}
$$


Using (6.2), one verifies easily that $\varphi(u, x)$ extends by continuity to $u=0$, and that

$$
\varphi(0, x)=\frac{\sinh (x / 2)}{x / 2} .
$$

The reader will have recognized in the right-hand side of (6.3) the inverse of the Hirzebruch polynomial $\hat{A}(x)$. In fact $\varphi(u, x)$ is a one-parameter deformation of $\hat{A}^{-1}(x)$.

Theorem 6.2. For $u \geq 0$ and $x \in \mathbb{C}$, the following identity holds:

$$
\varphi(u, x)=\prod_{k=1}^{+\infty}\left(1+\frac{i x}{2 k \pi}+\frac{u}{4 k^{2} \pi^{2}}\right)\left(1-\frac{i x}{2 k \pi}+\frac{u}{4 k^{2} \pi^{2}}\right) .
$$

Proof. We use the formula

$$
\sinh (y)=y \prod_{k=1}^{+\infty}\left(1+\frac{i y}{k \pi}\right)\left(1-\frac{i y}{k \pi}\right) .
$$

From (6.1) and (6.5), we get

$$
\begin{aligned}
\varphi(u, x)= & \prod_{k=1}^{+\infty}\left(1+i\left(\frac{x+\sqrt{x^{2}+4 u}}{4 k \pi}\right)\right)\left(1+i\left(\frac{x-\sqrt{x^{2}+4 u}}{4 k \pi}\right)\right) \\
& \times\left(1-i\left(\frac{x+\sqrt{x^{2}+4 u}}{4 k \pi}\right)\right)\left(1-i\left(\frac{x-\sqrt{x^{2}+4 u}}{4 k \pi}\right)\right) \\
= & \prod_{k=1}^{+\infty}\left(\left(1+\frac{i x}{4 k \pi}\right)^{2}+\frac{x^{2}+4 u}{16 k^{2} \pi^{2}}\right)\left(\left(1-\frac{i x}{4 k \pi}\right)^{2}+\frac{x^{2}+4 u}{16 k^{2} \pi^{2}}\right) \\
= & \prod_{k=1}^{+\infty}\left(1+\frac{i x}{2 k \pi}+\frac{u}{4 k^{2} \pi^{2}}\right)\left(1-\frac{i x}{2 k \pi}+\frac{u}{4 k^{2} \pi^{2}}\right) .
\end{aligned}
$$

Our theorem is proved.

(b) The multiplicative genus $\Psi(u, \cdot)$. Let $E$ be a real Euclidean vector space of even dimension $2 p$. Let $\mathscr{G}$ be the algebra of real antisymmetric matrices in $\operatorname{End}(E)$. Let $H_{E}$ be the Hilbert space

$$
H_{E}=\left\{f \in L^{2}([0,1] ; E) ; \int_{0}^{1} f d t=0\right\} .
$$

We identify $\left[0,1\left[\right.\right.$ with $S_{1}=\mathbb{R} / \mathbb{Z}$. In particular, the operator $J=\frac{d}{d t}$ acts naturally on distributions on $[0,1[$, so that for instance

$$
J t=1-\delta_{\{0\}} .
$$

Then

$$
H_{E}=\bigoplus_{k \in \mathbb{Z}^{*}}\left\{e^{2 \pi i k t}\right\}
$$


is the spectral decomposition of the Hilbert space $H_{E}$ with respect to the skewadjoint operator $J$. The inverse $J^{-1}$ of $J$ is a Hilbert-Schmidt operator acting on $H_{E}$.

Take $C \in \mathscr{G} \otimes_{R} \mathbb{C}$. Then $C$ acts naturally on $H_{E}$ as $f \in H_{E} \rightarrow C f \in$ $H_{E}$. For $u>0$, the operator $C J^{-1}+u J^{-2}$ is a Hilbert-Schmidt operator in $\operatorname{End}\left(H_{E}\right)$, which in general is not trace class. Since $C \in \mathscr{G} \otimes_{R} \mathbb{C}, \operatorname{Tr}[C]=0$. Therefore, we can define a generalized trace of $C J^{-1}+u J^{-2}$ on $H_{E}$ by the formula

$$
\operatorname{Tr}\left[C J^{-1}+u J^{-2}\right]=-\sum_{k \in \mathbb{Z}^{*}} \frac{u \operatorname{dim} E}{4 k^{2} \pi^{2}} .
$$

The operator $I_{H_{E}}-C J^{-1}-u J^{-2}$ does not possess a determinant in the sense of [Si2], since $C J^{-1}+u J^{-2}$ is not trace class. Still it has a normalized determinant. Namely, set

$$
\operatorname{det}\left(I_{H_{E}}-C J^{-1}-u J^{-2}\right)=\prod_{k \in \mathbb{Z}^{*}} \operatorname{det}\left(I_{E}-\frac{C}{2 i k \pi}+\frac{u I_{E}}{4 k^{2} \pi^{2}}\right) .
$$

The infinite product in the right-hand side of (6.9) converges, because as $|k| \rightarrow$ $+\infty$

$$
\operatorname{det}\left(I_{E}-\frac{C}{2 i k \pi}+\frac{u I_{E}}{4 k^{2} \pi^{2}}\right) \cong 1-\operatorname{Tr}\left[\frac{C}{2 i k \pi}\right]+O\left(\frac{1}{k^{2}}\right)
$$

and also $\operatorname{Tr}[C]=0$.

Since $C J^{-1}+u J^{-2}$ is Hilbert-Schmidt, the operator $I_{H_{E}}-C J^{-1}-u J^{-2}$ has a well-defined regularized determinant $\operatorname{det}_{2}\left(I-C J^{-1}-u J^{-2}\right)$ [Si2]. One easily verifies that although $C J^{-1}+u J^{-2}$ is not trace class, one still has the relation of [Si2, Theorem 6.2]

$$
\operatorname{det}_{2}\left(I_{H_{E}}-C J^{-1}-u J^{-2}\right)=\operatorname{det}\left(I_{H_{E}}-C J^{-1}-u J^{-2}\right) \exp \left\{\operatorname{Tr}\left[C J^{-1}+u J^{-2}\right]\right\} .
$$

Note that since $C$ is antisymmetric, for $k \in \mathbb{Z}^{*}$

$$
\operatorname{det}\left(I_{E}-\frac{C}{2 i k \pi}+\frac{u I_{E}}{4 k^{2} \pi^{2}}\right)=\operatorname{det}\left(I_{E}+\frac{C}{2 i k \pi}+\frac{u I_{E}}{4 k^{2} \pi^{2}}\right) .
$$

Therefore $\operatorname{det}\left(I_{H_{E}}-C J^{-1}-u J^{-2}\right)$ has a natural square root

$$
\operatorname{det}^{1 / 2}\left(I_{H_{E}}-C J^{-1}-u J^{-2}\right)=\prod_{k \in \mathbb{N}^{*}} \operatorname{det}\left(I_{E}-\frac{C}{2 i k \pi}+\frac{u I_{E}}{4 k^{2} \pi^{2}}\right) .
$$

If $B$ is a self-adjoint operator in End $E$, let $\sqrt{B}$ denote a square root of $B$ in the Banach algebra generated by $B$. In particular, if $F \subset E$ is an eigenspace for $B$, it is also an eigenspace for $\sqrt{B}$.

If $C \in \mathscr{G}$, then $C^{2}+4 u I_{E}$ is self-adjoint for $u \geq 0$. 
Definition 6.3. For $u>0$ and $C \in \mathscr{G}$, set

$$
\Psi(u, C)=\frac{1}{u^{(\operatorname{dim} E) / 2}} \operatorname{det}\left(2 \sinh \left(\frac{C+\sqrt{C^{2}+4 u I_{E}}}{4}\right)\right) .
$$

One verifies that $\Psi(u, C)$ does not depend on the square root $\sqrt{C^{2}+4 u I_{E}}$. If $C$ has eigenvalues $\pm x_{1}, \ldots, \pm x_{p}$ one deduces from (6.1) that

$$
\Psi(u, C)=\prod_{1}^{p} \varphi\left(u, x_{i}\right)
$$

If $C$ has eigenvalues $\pm x_{1}, \ldots, \pm x_{p}$, the Hirzebruch polynomial $\widehat{A}(C)$ is given by

$$
\widehat{A}(C)=\prod_{1}^{p} \frac{x_{i} / 2}{\sinh \left(x_{i} / 2\right)}
$$

Using (6.3), we find in particular that $\Psi(u, C)$ can be extended by continuity at $u=0$ and that

$$
\Psi(0, C)=\hat{A}^{-1}(C) .
$$

Also from Theorem 6.2 and from (6.13) and (6.14), we find that

$$
\Psi(u, C)=\operatorname{det}^{1 / 2}\left(I_{H_{E}}-C J^{-1}-u J^{-2}\right) .
$$

Note that $\Psi(u, C)$ is an analytic function of $C$ and so is also well defined for $C \in \mathscr{G} \otimes_{R} \mathbb{C}$.

(c) A computation of certain finite-dimensional supertraces. We now use the same notation as in $\S 1$.

Let $C$ be a skew-adjoint element of $\operatorname{End}(V)$. In the sequel, $\operatorname{Tr}[C]$ denotes the trace of $C \in \operatorname{End}(V) . C$ acts as a derivation of $\Lambda\left(V^{*}\right)$. More precisely if $e_{1}, \ldots, e_{2 n}$ is an orthonormal base of $V_{R}, C$ acts on $\Lambda\left(V^{*}\right)$ as $\widehat{C}$ given by

$$
\widehat{C}=\frac{1}{4}\left\langle C e_{i}, e_{j}\right\rangle \hat{c}\left(e_{i}\right) \hat{c}\left(e_{i}\right)-\frac{1}{2} \operatorname{Tr}[C] .
$$

We identify $C$ with the corresponding real antisymmetric element in $\operatorname{End}\left(V_{R}\right)$ (whose real trace is of course zero). Recall that the operator $S$ was defined in Definition 1.3.

The algebra $\Lambda\left(V_{R}^{*}\right)$ is naturally $\mathbb{Z}_{2}$ graded. Let $\operatorname{Tr}_{s}$ denote the corresponding supertrace on $\operatorname{End}\left(\Lambda\left(V_{R}^{*}\right)\right)$.

Theorem 6.4. For any $u \geq 0$, the following identity holds:

$$
\operatorname{Tr}_{s}[\exp (-\sqrt{u} S-\widehat{C})]=u^{\operatorname{dim} V} \Psi(u, C) \exp \left\{\frac{1}{2} \operatorname{Tr}[C]\right\} .
$$

Proof. We may and we will assume that the matrix of $C$ with respect to the base $e_{1}, \ldots, e_{2 n}$ has diagonal blocks given by

$$
\left[\begin{array}{cc}
0 & i x_{j} \\
-i x_{j} & 0
\end{array}\right] \quad(1 \leq j \leq n)
$$


Set

$$
\hat{c}(C)=\frac{1}{4}\left\langle C e_{i}, e_{i}\right\rangle \hat{c}\left(e_{i}\right) \hat{c}\left(e_{j}\right) .
$$

By (6.18) and (6.20), we find that

$$
\operatorname{Tr}_{s}[\exp (-\sqrt{u} S-\widehat{C})]=\exp \left\{\frac{1}{2} \operatorname{Tr}[C]\right\} \operatorname{Tr}_{s}[\exp (-\sqrt{u} S-\hat{c}(C))]
$$

Consider the vector space $V_{R} \oplus V_{R}$, which we equip with the metric which is the orthogonal sum of the given metrics on both copies of $V_{R}$. Let $\tilde{c}\left(V_{R} \oplus V_{R}\right)$ be the Clifford algebra of $V_{R} \oplus V_{R}$ and let $B$ be a real antisymmetric matrix in End $\left(V_{R} \oplus V_{R}\right)$. If $f_{1}, \ldots, f_{4 n}$ is an orthonormal base of $V_{R} \oplus V_{R}$, set

$$
\tilde{c}(B)=\frac{1}{4}\left\langle B f_{i}, f_{j}\right\rangle \tilde{c}\left(f_{i}\right) \tilde{c}\left(f_{j}\right) \text {. }
$$

We now assume that $B \in \operatorname{End}\left(V_{R} \oplus V_{R}\right) \otimes_{R} \mathbb{C}$ is given in matrix form by

$$
B=\left[\begin{array}{cc}
0 & -i \sqrt{u} \mathbf{J} \\
-i \sqrt{u} \mathbf{J} & -C
\end{array}\right] \text {. }
$$

Let $\operatorname{Tr}_{s}[\exp (\tilde{c}(B))]$ denote the supertrace of $\exp (\tilde{c}(B))$ acting on the spinors of $V_{R} \oplus V_{R}$. We claim that

$$
\operatorname{Tr}_{s}[\exp (-\sqrt{u} S-\hat{c}(C))]=(-1)^{\operatorname{dim} V} \operatorname{Tr}_{s}[\exp (\tilde{c}(B))]
$$

Let $\tau$ and $\tau^{\prime}$ be the operators defining the $\mathbb{Z}_{2}$ grading on $\Lambda\left(\bar{N}^{*}\right)$ and $\Lambda\left(N^{*}\right)$. One verifies easily that

$$
\begin{gathered}
\tau=i^{\operatorname{dim} V} c\left(e_{1}\right) \cdots c\left(e_{2 n}\right), \\
\tau^{\prime}=(-i)^{\operatorname{dim} V} \hat{c}\left(e_{1}\right) \cdots \hat{c}\left(e_{2 n}\right) .
\end{gathered}
$$

Using (6.22) and (6.23) and the fact that $\tau$ and $\tau^{\prime}$ are the only monomials (up to a multiplicative constant) with a nonzero supertrace when acting on $\Lambda\left(\bar{N}^{*}\right)$ and $\Lambda\left(N^{*}\right)$ [AB, p. 484], we deduce (6.24).

We now calculate $\operatorname{Tr}_{s}[\exp (\tilde{c}(B))]$ using formula (5.6). If $\lambda$ is an eigenvalue of $B$ and if $\left(X, X^{\prime}\right)$ is a corresponding nonzero eigenvector, then

$$
\begin{gathered}
-i \sqrt{u} \mathbf{J} X^{\prime}=\lambda X, \\
-i \sqrt{u} \mathbf{J} X-C X^{\prime}=\lambda X^{\prime} .
\end{gathered}
$$

Using (6.26) and the fact that $\mathbf{J}^{2}=-1$, we find that for $u \neq 0, \lambda$ is nonzero, $X^{\prime}$ is nonzero, and moreover

$$
(C-u / \lambda+\lambda) X^{\prime}=0 .
$$

Conversely, if (6.27) holds with $\lambda, X^{\prime} \neq 0$, then $\left(-i \sqrt{u} \mathbf{J} X^{\prime} / \lambda, X^{\prime}\right)$ is a nonzero eigenvector of $B$ associated with the eigenvalue $\lambda$. By (6.27), we find that $X^{\prime}$ is an eigenvector of $C$ associated with an eigenvalue $x$ such that

$$
\lambda^{2}+\lambda x-u=0
$$

Therefore,

$$
\lambda=\frac{-x \pm \sqrt{x^{2}+4 u}}{2}
$$


For $u \neq 0$, the roots of (6.28) are nonzero. Since the matrix $B$ is not real, we cannot use formula (5.6). However, we can analytically continue $B$ to $u<0$. $B$ is then real, and we may safely use formula (5.6). Using (6.29), we find easily that if $x_{1}, \ldots, x_{n}$ are the eigenvalues of $C$ acting on $V$, then for $u \neq 0$

$$
\operatorname{Tr}_{s}[\exp (\tilde{c}(B))]=\prod_{1}^{n} 4 \sinh \left(\frac{x_{k}+\sqrt{x_{k}^{2}+4 u}}{4}\right) \sinh \left(\frac{x_{k}-\sqrt{x_{k}^{2}+4 u}}{4}\right)
$$

From (6.1) and (6.30), we get

$$
\operatorname{Tr}_{s}[\exp (\tilde{c}(B))]=(-u)^{\operatorname{dim} V} \prod_{1}^{n} \varphi\left(u, x_{i}\right)
$$

From (6.21), (6.24), and (6.31), we get

$$
\operatorname{Tr}_{s}[\exp (-\sqrt{u} S-\widehat{C})]=\exp \left\{\frac{1}{2} \operatorname{Tr}[C]\right\} u^{\operatorname{dim} V} \prod_{1}^{n} \varphi\left(u, x_{i}\right) .
$$

(6.19) now follows from (6.15) and (6.32) for $u<0$, and by analytic continuation for $u \geq 0$.

Remark 6.5. The complex structure on $V$ plays an unessential role here. As is clear from (6.22) and (6.29), (6.30) is in fact easily extendable to any arbitrary real Euclidean oriented even-dimensional vector space. We should only replace $S$ by $\frac{\sqrt{-1}}{2} \sum_{1}^{2 n} c\left(e_{i}\right) \hat{c}\left(e_{i}\right)$.

(d) A computation of the trace of certain harmonic oscillators with a magnetic field. We use the same notation as before. Recall that $C$ is identified with a real antisymmetric matrix in End $\left[V_{R}\right]$. For $u \geq 0$, set

$$
\mathscr{L}_{u}=-\frac{1}{2} \sum_{1}^{2 n}\left(\nabla_{e_{i}}+\frac{1}{2}\left\langle C Y, e_{i}\right\rangle\right)^{2}+\frac{1}{2} u|Y|^{2}+\frac{1}{2} \operatorname{Tr}[C] .
$$

If $x_{1}, \ldots, x_{n}$ are the eigenvalues of $C$, set $|C|=\sup _{1 \leq i \leq n}\left|x_{i}\right|$.

Theorem 6.6. Assume that $u$ is positive. Then if $|C|<2 \pi$, the operator $\exp \left(-\mathscr{L}_{u}\right)$ is trace class and moreover

$$
\operatorname{Tr}\left[\exp \left(-\mathscr{L}_{u}\right)\right]=\frac{\exp \left(-\frac{1}{2} \operatorname{Tr}[C]\right)}{u^{\operatorname{dim} V} \Psi(u, C)}
$$

Proof. Clearly,

$$
\mathscr{L}_{u}=-\frac{1}{2} \Delta-\frac{1}{2} \nabla_{C Y}+\frac{1}{2}\left\langle\left(C^{2} / 4+u I_{E}\right) Y, Y\right\rangle+\frac{1}{2} \operatorname{Tr}[C] .
$$

Set

$$
\mathscr{M}_{u}=-\frac{1}{2} \Delta+\frac{1}{2}\left\langle\left(C^{2} / 4+u I_{E}\right) Y, Y\right\rangle .
$$

Then since $C \in$ End $V_{R}$ is antisymmetric, we find that

$$
\left[\mathscr{M}_{u}, \nabla_{C Y}\right]=0 \text {. }
$$


Assume that $C^{2} / 4+u I_{E}>-\pi^{2}$. Let $Q$ be an arbitrary square root of $C^{2} / 4+u I_{E}$ in the Banach algebra generated by $C$. By Mehler's formula [GlJ, Theorem 1.5.10], we know that for $0<s \leq 1$, the kernel $p_{s}\left(Y, Y^{\prime}\right)$ associated with the operator $\exp \left(-s \mathscr{M}_{u}\right)$ is given by

$$
\begin{aligned}
& p_{s}\left(Y, Y^{\prime}\right)=\frac{1}{s^{\operatorname{dim} V}} \operatorname{det}^{1 / 2}\left(\frac{s Q}{\sinh (s Q)}\right) \\
& \times \exp \left\{-\frac{1}{2 s}\left\langle\frac{s Q}{\tanh (s Q)} Y, Y\right\rangle-\frac{1}{2 s}\left\langle\frac{s Q}{\tanh (s Q)} Y^{\prime}, Y^{\prime}\right\rangle\right. \\
& \left.+\frac{1}{s}\left\langle\frac{s Q}{\sinh (s Q)} Y, Y^{\prime}\right\rangle\right\} \text {. }
\end{aligned}
$$

From (6.36) and (6.37), we deduce that if $q_{s}\left(Y, Y^{\prime}\right)$ denotes the smooth kernel associated with the operator $\exp \left(-s \mathscr{L}_{u}\right)$ for $0<s \leq 1$, then

$$
q_{s}\left(Y, Y^{\prime}\right)=p_{s}\left(e^{s C / 2} Y, Y^{\prime}\right) \exp \left(-\frac{1}{2} s[\operatorname{Tr} C]\right) .
$$

From (6.37) and (6.38), we find in particular that

$$
\begin{aligned}
q_{s}(Y, Y)= & \frac{1}{s^{\operatorname{dim} V}} \operatorname{det}^{1 / 2}\left(\frac{s Q}{\sinh (s Q)}\right) \\
& \times \exp \left\{-\frac{1}{s}\left\langle\frac{s Q}{\sinh (s Q)}\left(\cosh (s Q)-e^{s C / 2}\right) Y, Y\right\rangle-\frac{1}{2} s \operatorname{Tr}[C]\right\} .
\end{aligned}
$$

Since $C$ is antisymmetric, we have the obvious (6.40)

$$
\begin{aligned}
& \left\langle\frac{s Q}{\sinh (s Q)}\left(\operatorname{ch}(s Q)-e^{s C / 2}\right) Y, Y\right\rangle \\
& \quad=\left\langle\frac{s Q}{\sinh (s Q)}\left(\cosh (s Q)-\cosh \left(\frac{s C}{2}\right)\right) Y, Y\right\rangle \\
& \quad=\frac{1}{2}\left\langle\left(\frac{s Q}{\sinh (s Q)}\right) 2 \sinh \left(\frac{s C+2 s Q}{4}\right) Y, 2 \sinh \left(\frac{s C+2 s Q}{4}\right) Y\right\rangle .
\end{aligned}
$$

Also one easily verifies that if $|C|<2 \pi$, the operator $\sinh ((s C+2 s Q) / 4)$ is invertible for $s \leq 1$ and $u>0$. From (6.39) and (6.40), we find that if $|C|<2 \pi$, for $0<s \leq 1$

$$
\int_{V_{R}} q_{s}(Y, Y) \frac{d v(Y)}{(2 \pi)^{\operatorname{dim} V}}=\exp \left(-\frac{s}{2} \operatorname{Tr}[C]\right) \frac{1}{|\operatorname{det}(2 \sinh ((s C+2 s Q) / 4))|}
$$

Comparing with (6.14), we find that

$$
\int_{V_{R}} q_{s}(Y, Y) \frac{d v(Y)}{(2 \pi)^{\operatorname{dim} V}}=\frac{\exp \left(-\frac{s}{2} \operatorname{Tr}[C]\right)}{\left(s^{2} u\right)^{\operatorname{dim} V}\left|\Psi\left(s^{2} u, s C\right)\right|}
$$

Since $\left(q_{s}\right), s \in[0,1]$, forms a semigroup and the $q_{s}$ are positive, we deduce from (6.42) that for $0<s \leq \frac{1}{2}$, the operators $\exp \left(-s \mathscr{L}_{u}\right)$ are Hilbert-Schmidt. Since $\exp \left(-\mathscr{L}_{u}\right)=\exp \left(-\mathscr{L}_{u} / 2\right) \exp \left(-\mathscr{L}_{u} / 2\right)$, the operator $\exp \left(-\mathscr{L}_{u}\right)$ is trace 
class. Using (6.42), we obtain

$$
\operatorname{Tr}\left[\exp \left(-\mathscr{L}_{u}\right)\right]=\frac{\exp \left(-\frac{1}{2} \operatorname{Tr}[C]\right)}{u^{\operatorname{dim} V}|\Psi(u, C)|}
$$

Now if $x \in \sqrt{-1} \mathbb{R},|x|<2 \pi$, it is clear from (6.4) that $\varphi(u, x)>0$. From (6.15), we deduce that if $|C|<2 \pi$, then $\Psi(u, C)$ is positive. (6.34) now follows from (6.43).

Remark 6.7. It is also possible to prove directly that

$$
\operatorname{Tr}\left[\exp \left(-\mathscr{L}_{u}\right)\right]=\frac{\exp \left(-\frac{1}{2} \operatorname{Tr}[C]\right)}{u^{\operatorname{dim} V} \operatorname{det}^{1 / 2}\left(I_{H_{V}}-C J^{-1}-u J^{-2}\right)}
$$

by using infinite-dimensional Gaussian integration, from which (6.34) follows via (6.17). This point of view will be developed more in $\S 7$.

Remark 6.8. The striking similarity of formulas (6.19) and (6.34) is of course no accident. In fact note that from (6.19) and (6.34), we get

$$
\operatorname{Tr}_{s}\left[\exp \left\{\frac{1}{2} \sum_{1}^{2 n}\left(\nabla_{e_{i}}+\frac{1}{2}\left\langle C Y, e_{i}\right\rangle\right)^{2}-\frac{u|Y|^{2}}{2}-\frac{1}{2} \operatorname{Tr}[C]-\sqrt{u} S-\widehat{C}\right\}\right]=1 .
$$

Observe that the operators $\bar{\partial}+\sqrt{-u} i_{y}+\bar{\partial}^{*}-\sqrt{-u} i_{y}^{*}$ are invariant under complex unitary transformations. In particular, $e^{-C}$ acts naturally on the graded kernel of this operator. In view of Proposition 1.4 and Theorem 3.10, an easy adaptation of the results of [B11, Theorems 1.6 and 1.9] shows that the left-hand side of (6.45) is a nonclassical formula for the supertrace of the action of $e^{-C}$ on $\operatorname{Ker}\left(\bar{\partial}+\sqrt{-1} i_{y}+\bar{\partial}^{*}-\sqrt{-1} i_{y}^{*}\right)$, i.e., for a Lefchetz number. Now by Theorem 1.6, we immediately find that $e^{-C}$ acts trivially on the one-dimensional vector space spanned by $\beta$. This entirely explains formula (6.45). In the context of Theorem $2.2,(6.45)$ is directly related to the fact that $\beta$ is a parallel section of the trivial line bundle $\mu$. Also, by Remark 1.9, we know that the $L_{2}$ cohomology of the complex $\left(\Gamma^{0}, \bar{\partial}+\sqrt{-u} i_{y}\right)$ is the same as the cohomology of $\{0\}$. Tautologically, $e^{-C}$ acts as the identity map on the one-dimensional cohomology of $\{0\}$. We then find that formula (6.45) is not too surprising after all.

\section{AN EXPLICIT EVALUATION OF THE GENERALIZED SUPERTRACES}

In this section, we explicitly evaluate the forms $\operatorname{Tr}_{s}\left[\exp \left(-\mathscr{B}_{u}^{2}+b N_{H}\right)\right]$ in terms of certain infinite determinants. Although the formulas may look complicated, they are strikingly similar to formulas we obtain in $\S 9$ in finite-dimensional equivariant intersection theory. We also rederive the asymptotic result of $\S 4$ which says that as $u \rightarrow 0$

$$
\operatorname{Tr}_{s}\left[\exp \left(-\mathscr{B}_{u}^{2}\right)\right]=\frac{\operatorname{Td}\left(-R^{M}\right)}{\operatorname{Td}\left(-R^{N}\right)}+O(u),
$$


and we prove that as $u \rightarrow \infty$

$$
\operatorname{Tr}_{s}\left[\exp \left(-\mathscr{B}_{u}^{2}\right)\right]=\operatorname{Td}\left(-R^{L}\right)+O\left(\frac{1}{\sqrt{u}}\right) .
$$

Both proofs make use of our explicit formula for $\operatorname{Tr}_{s}\left[\exp \left(-\mathscr{B}_{u}^{2}\right)\right]$.

This section is organized as follows. In (a), we give a path integral representation of $\operatorname{Tr}_{s}\left[P_{1}^{x, u, b}(Y, Y)\right]$. In (b) we express $\operatorname{Tr}_{s}\left[\exp \left(-\mathscr{B}_{u}^{2}+b N_{H}\right)\right]$ in terms of certain infinite determinants and we reobtain the asymptotics of this form as $u \rightarrow 0$. Finally in (c), we calculate the asymptotics of $\operatorname{Tr}_{s}\left[\exp \left(-\mathscr{B}_{u}^{2}+b N_{H}\right)\right]$ as $u \rightarrow+\infty$.

The results of this section play a crucial role in the construction of $\S 8$.

We make the same assumptions and we use the same notations as in $\S \S 3-6$.

(a) A path integral formula for $\operatorname{Tr}_{s}\left[P_{1}^{x, u, b}(Y, Y)\right]$. Let $I_{N} \in \operatorname{End}(N)$ be the identity map of $N$. Let $I_{N}^{\prime} \in \operatorname{End}\left(N_{R} \otimes_{R} \mathbb{C}\right)$ be the linear map which acts by multiplication by 1 on $N$ and by -1 on $\bar{N}$. Equivalently, if $\mathbf{J}_{N}$ is the complex structure of $N_{R}$, then

$$
I_{N}^{\prime}=-i \mathbf{J}_{N}
$$

If $E$ is a real vector space, $H_{E}$ was defined in (6.7). If $V$ is a complex vector space, we use the notation $H_{V}$ instead of $H_{V_{R}}$.

Definition 7.1. For $Y \in N_{R}$, let $Q_{(Y, Y)}$ be the probability law on $\mathscr{C}\left([0,1] ; M_{R}\right)$ of the Brownian bridge $t \in[0,1] \rightarrow Y_{t}$, with $Y_{0}=Y_{1}=Y$.

Note that under $Q_{(Y, Y)}, t \in[0,1] \rightarrow P^{L} Y_{t} \in L_{R}$ is a Brownian bridge in $L_{R}$, with $P^{L} Y_{0}=P^{L} Y_{1}=0$.

In the sequel, for $u>0, b \in \mathbb{C}$, and $|\operatorname{Im} b|<2 \pi$, we will consider expressions which are functions of $Y \in \mathscr{C}\left([0,1] ; M_{R}\right)$ of the type

$$
\Psi\left(u, R^{N}+b I_{N}^{\prime}\right) \exp \left\{-\frac{u}{2}\left\langle P^{L} A\left(J^{2}-\left(R^{N}+b I_{N}^{\prime}\right) J-u I_{H_{N}}\right)^{-1} J A P^{L} Y, Y\right\rangle\right\} .
$$

Note that since the map $t \in[0,1] \rightarrow Y_{t}$ is continuous, $Y$ defines an element of $L_{2}\left([0,1] ; M_{R}\right)$. For $u>0, b \in \mathbb{C}$, and $|\operatorname{Im} b|<2 \pi$,

$$
P^{L} A\left(J^{2}-\left(R^{N}+b I_{N}^{\prime}\right) J-u I_{H_{N}}\right)^{-1} J A P^{L}
$$

is an even form on $B$ with values in the set of the continuous operators acting on $L_{2}\left([0,1] ; N_{R}\right)$. Therefore the expression (7.2) makes sense. Incidentally note that, with the conventions of (5.17) and (6.17), (7.2) also makes sense for arbitrary $b \in \mathbb{C}$. In the sequel, the expressions we will consider are analytic in $b \in \mathbb{C}$.

Also note that, when writing an identity of the kind

$$
A(b)=E^{Q_{(Y, Y)}}[B(b)],
$$

$A(b)$ and $B(b)$ will be analytic functions of $b$, and $|\operatorname{Im} b|<2 \pi$. It will always be understood that differentiation under the expectation sign is feasible, so that 
for any $k \in \mathbb{N}$

$$
A^{(k)}(b)=E^{Q_{(Y, Y)}}\left[B^{(k)}(b)\right] .
$$

Theorem 7.2. For any $u>0, x \in B, Y \in N_{R, x}, b \in \mathbb{C}$, and $|\operatorname{Im} b|<2 \pi$ the following identity holds:

$$
\begin{aligned}
\operatorname{Tr}_{s}\left[P_{1}^{x, u, b}(Y, Y)\right] & =u^{\operatorname{dim} N} \Psi\left(u, R^{N}+b I_{N}^{\prime}\right) \exp \left\{-\frac{1}{2} \operatorname{Tr}\left[R^{L}\right]+\frac{1}{2} b \operatorname{dim} N\right\} \\
\times E^{Q_{(Y, Y)}}[\exp & \left\{\frac{1}{2} \int_{0}^{1}\left\langle R^{M} Y, d Y\right\rangle-\frac{u}{2} \int_{0}^{1}\left|P^{N} Y\right|^{2} d t\right. \\
& \left.\left.-\frac{u}{2}\left\langle P^{L} A\left(J^{2}-\left(R^{N}+b I_{N}^{\prime}\right) J-u I_{H_{N}}\right)^{-1} J A P^{L} Y, Y\right\rangle\right\}\right] .
\end{aligned}
$$

Proof. We use the notation of the proof of Theorem 4.1. Let $U_{s}$ be given by equation (4.6). Using equation (4.11), we find that

$$
\begin{aligned}
& \operatorname{Tr}_{s}\left[P_{1}^{x, u, b}(Y, Y)\right]=\exp \left\{-\frac{1}{2} \operatorname{Tr}\left[R^{M}\right]\right\} \\
& \times E^{Q_{(Y, Y)}}\left[\exp \left\{\frac{1}{2} \int_{0}^{1}\left\langle R^{M} Y, d Y\right\rangle-\frac{u}{2} \int_{0}^{1}\left|P^{N} Y\right|^{2} d t\right\} \operatorname{Tr}_{s}\left[U_{1}\right]\right] .
\end{aligned}
$$

We now calculate $\operatorname{Tr}_{s}\left[U_{1}\right]$. One verifies easily that

$$
N_{H}=-\widehat{I_{N}^{\prime}} \text {. }
$$

Let $\mathbf{B} \in \Lambda\left(T_{R}^{*} B\right) \widehat{\otimes} \operatorname{End}\left(N_{R} \oplus N_{R}\right)$ be given in matrix form by

$$
\mathbf{B}=\left[\begin{array}{cc}
0 & -i \sqrt{u} \mathbf{J} \\
-i \sqrt{u} \mathbf{J} & -R^{N}-b I_{N}^{\prime}
\end{array}\right] .
$$

We now temporarily consider $A P^{L}$ as a one-form on $B$ with values in homomorphisms from $L_{R}$ into the second copy of $N_{R}$ in $N_{R} \oplus N_{R}$. Using (4.6) and Theorem 5.1, we find that

$$
\begin{aligned}
\operatorname{Tr}_{s}\left[U_{1}\right]= & \operatorname{Tr}_{s}\left[\exp \left(-\sqrt{u} S-\widehat{R}^{N}-b \widehat{I}_{N}^{\prime}\right)\right] \\
& \times \exp \left\{-\frac{u}{2}\left\langle\left(\frac{d}{d t}+\mathbf{B}\right)^{-1} A P^{L} Y, A P^{L} Y\right\rangle\right\} .
\end{aligned}
$$

By Theorem 6.4, we know that

$$
\begin{aligned}
& \operatorname{Tr}_{s}\left[\exp \left(-\sqrt{u} S-\widehat{R}^{N}-b \widehat{I}_{N}^{\prime}\right)\right] \\
& \quad=u^{\operatorname{dim} N} \psi\left(u, R^{N}+b I_{N}^{\prime}\right) \exp \left\{\frac{1}{2} \operatorname{Tr}\left[R^{N}\right]+\frac{b}{2} \operatorname{dim} N\right\} .
\end{aligned}
$$

Let now $t \in S_{1} \rightarrow K_{t} \in N_{R}$ be a smooth function. We consider $K_{t}$ as living in the second copy of $N_{R}$ in $N_{R} \oplus N_{R}$. Set $C=R^{N}+b I_{N}^{\prime}$. To calculate $\left(\frac{d}{d t}+\mathbf{B}\right)^{-1} K_{t}$, we must solve the differential equation

$$
\frac{d \varphi}{d t}-i \sqrt{u} \mathbf{J} \psi=0, \quad \frac{d \psi}{d t}-i \sqrt{u} \mathbf{J} \varphi-C \psi=K
$$


with periodic boundary conditions. From (7.11), we find that

$$
\frac{d^{2} \psi}{d t^{2}}-C \frac{d \psi}{d t}-u \psi=\frac{d K}{d t}
$$

and $\psi$ should also be periodic, with periodic first derivatives. From (7.12), we get

$$
\psi=\left(J^{2}-C J-u I_{H_{N}}\right)^{-1} J K .
$$

From (7.9)-(7.13), we find that

$$
\begin{aligned}
\operatorname{Tr}_{s}\left[U_{1}\right]= & u^{\operatorname{dim} N} \Psi\left(u, R^{N}+b I_{N}^{\prime}\right) \exp \left\{\frac{1}{2} \operatorname{Tr}\left[R^{N}\right]+\frac{b}{2} \operatorname{dim} N\right\} \\
& \times \exp \left\{-\frac{u}{2}\left\langle P^{L} A\left(J^{2}-\left(R^{N}+b I_{N}^{\prime}\right) J-u I_{H_{N}}\right)^{-1} J A P^{L} Y, Y\right\rangle\right\} .
\end{aligned}
$$

Also

$$
\operatorname{Tr}\left[R^{M}\right]=\operatorname{Tr}\left[R^{L}\right]+\operatorname{Tr}\left[R^{N}\right] .
$$

Using (7.6), (7.14), and (7.15), we get (7.5). The fact that (7.5) can be differentiated as many times as needed in the variable $b$ is an easy consequence of the estimates in Theorem 4.1 and is left to the reader.

(b) Generalized supertraces and infinite determinants. Set

$$
\mathscr{E}=-R^{M} J^{-1}-u P^{L} A\left(J^{2}-\left(R^{N}+b I_{N}^{\prime}\right) J-u I_{H_{N}}\right)^{-1} A P^{L} J^{-1}-u P^{N} J^{-2}
$$

Then one verifies easily that $\mathscr{E}$ is an even form on $B$ taking values in HilbertSchmidt operators acting on $H_{M}$. In general $\mathscr{E}$ does not take values in trace class operators, because the operator $J^{-1}$ is not trace class.

We now proceed as in $\S 6$. In fact $\mathscr{E}$ has a normalized trace. Namely for $k \in \mathbb{Z}^{*}$, let $\mathscr{E}_{k}$ be the restriction of $\mathscr{E}$ to the eigenspace $M_{R} \otimes\left\{e^{2 i \pi k t}\right\} \cong M_{R}$ of $J$. Since $R^{M}$ takes values in antisymmetric elements of $\operatorname{End}\left(M_{R}\right)$, then $\operatorname{Tr}^{M_{R}}\left[R^{M}\right]=0$. One then easily deduces that as $|k| \rightarrow+\infty$

$$
\operatorname{Tr}\left[\mathscr{E}_{k}\right]=O\left(1 / k^{2}\right) \text {. }
$$

We now define the formal trace of $\mathscr{E}$ by

$$
\operatorname{Tr}[\mathscr{E}]=\sum_{k \in \mathbb{Z}^{*}} \operatorname{Tr}\left[\mathscr{E}_{k}\right]
$$

Similarly, since $\operatorname{Tr}^{M_{R}}\left[R^{M}\right]=0$, one verifies that as $|k| \rightarrow \infty$

$$
\operatorname{det}\left(I_{M_{R}}+\mathscr{E}_{k}\right)-1=O\left(1 / k^{2}\right) \text {. }
$$

We define the normalized determinant of $I_{H_{M}}+\mathscr{E}$, denoted $\operatorname{det}\left(I_{H_{M}}+\mathscr{E}\right)$ by the formula

$$
\operatorname{det}\left(I_{H_{M}}+\mathscr{E}\right)=\prod_{k \in \mathbb{Z}^{*}} \operatorname{det}\left(I_{M_{R}}+\mathscr{E}_{k}\right)
$$


Let $\tilde{\mathscr{E}}_{k}$ denote the transpose of $\mathscr{E}_{k}$. Then one verifies easily that

$$
\tilde{\mathscr{E}}_{k}=\mathscr{E}_{-k}
$$

From (7.20), we deduce that

$$
\prod_{k=1}^{\infty} \operatorname{det}\left(I_{M_{R}}+\mathscr{C}_{k}\right)=\prod_{k=1}^{\infty} \operatorname{det}\left(I_{M_{R}}+\mathscr{E}_{-k}\right) \text {. }
$$

Therefore $\prod_{k=1}^{\infty} \operatorname{det}\left(I_{M_{R}}+\mathscr{E}_{k}\right)$ is a natural square root of $\operatorname{det}\left(I_{H_{M}}+\mathscr{E}\right)$. We will use the notation

$$
\operatorname{det}^{1 / 2}\left(I_{H_{M}}+\mathscr{E}\right)=\prod_{k=1}^{\infty} \operatorname{det}\left(I_{M_{R}}+\mathscr{E}_{k}\right) .
$$

If we formally make $R^{M}=0$ and $A=0$ in $\mathscr{E}$, we get

$$
\operatorname{det}\left(I_{H_{M}}+\mathscr{E}\right)=\left(\frac{\sinh (\sqrt{u} / 2)}{\sqrt{u} / 2}\right)^{2 \operatorname{dim} N_{R}}
$$

so that $\operatorname{det}^{1 / 2}\left(I_{H_{M}}+\mathscr{E}\right)$ is positive and is equal to $(\sinh (\sqrt{u} / 2) / \sqrt{u} / 2)^{\operatorname{dim} N_{R}}$. In the general case, $\operatorname{det}^{1 / 2}\left(I_{H_{M}}+\mathscr{E}\right)$ can be calculated by Taylor expansion near $R^{M}, A=0$ and coincides with (7.22). It is of utmost importance here to observe that, in $\operatorname{det}^{1 / 2}\left(I_{H_{M}}+\mathscr{E}\right), I_{H_{M}}+\mathscr{E}$ is considered as an operator acting on the real Hilbert space $H_{M}$.

With the same conventions, we deduce from (6.17) that

$$
\operatorname{det}^{1 / 2}\left(I_{H_{N}}-\left(R^{N}+b I_{N}^{\prime}\right) J^{-1}-u J^{-2}\right)=\Psi\left(u, R^{N}+b I_{N}^{\prime}\right) .
$$

Theorem 7.3. For any $u>0$ and $b \in \mathbb{C}$, the following identity holds:

$$
\begin{aligned}
\operatorname{Tr}_{s}\left[\exp \left(-\mathscr{B}_{u}^{2}+b N_{H}\right)\right] & \\
= & \exp \left\{-\frac{1}{2} \operatorname{Tr}[L]+\frac{b}{2} \operatorname{dim} N\right\} \operatorname{det}^{1 / 2}\left(I_{H_{N}}-\left(R^{N}+b I_{N}^{\prime}\right) J^{-1}-u J^{-2}\right) \\
\times & \times \operatorname{det}^{1 / 2}\left(I_{H_{M}}-R^{M} J^{-1}-u P^{L} A\left(J^{2}-\left(R^{N}+b I_{N}^{\prime}\right) J-u I_{H_{N}}\right)^{-1}\right. \\
& \left.\left.\times A P^{L} J^{-1}-u P^{N} J^{-2}\right)\right]^{-1} .
\end{aligned}
$$

Proof. By (4.32) and (7.5), we find that

$$
\begin{aligned}
\operatorname{Tr}_{s}\left[\operatorname { e x p } \left(-\mathscr{B}_{u}^{2}\right.\right. & \left.\left.+b N_{H}\right)\right] \\
= & u^{\operatorname{dim} N} \exp \left\{-\frac{1}{2} \operatorname{Tr}\left[R^{L}\right]+\frac{b}{2} \operatorname{dim} N\right\} \Psi\left(u, R^{N}+b I_{N}^{\prime}\right) \\
& \times \int_{N_{R}} E^{Q_{(Y, Y)}}\left[\operatorname { e x p } \left\{\frac{1}{2} \int_{0}^{1}\left\langle R^{M} Y, d Y\right\rangle-\frac{u}{2} \int_{0}^{1}\left|P^{N} Y\right|^{2} d t\right.\right. \\
& \left.\left.-\frac{u}{2}\left\langle P^{L} A\left(J^{2}-\left(R^{N}+b I_{N}^{\prime}\right) J-u I_{H_{N}}\right)^{-1} J A P^{L} Y, Y\right\rangle\right\}\right] \frac{d v_{N}(Y)}{(2 \pi)^{\operatorname{dim} N}}
\end{aligned}
$$


Take $f \in L_{2}\left([0,1] ; M_{R}\right)$. We first calculate the expression (7.26)

$$
I=\int_{N_{R}} E^{Q_{(Y, Y)}}\left[\exp \left\{\int_{0}^{1}\left\langle f, d Y-\sqrt{u} P^{N} Y d t\right\rangle-\frac{u}{2} \int_{0}^{1}\left|P^{N} Y\right|^{2} d t\right\}\right] \frac{d v_{N}(Y)}{(2 \pi)^{\operatorname{dim} N}} .
$$

Here we follow Yor $[Y]$. Namely, by using the Ito calculus, we know that

$$
\begin{aligned}
\int_{0}^{1}\langle f & \left., d Y-\sqrt{u} P^{N} Y d t\right\rangle-\frac{u}{2} \int_{0}^{1}\left|P^{N} Y\right|^{2} d t \\
= & \int_{0}^{1}\left\langle f+\sqrt{u} P^{N} Y, \delta Y\right\rangle-\frac{1}{2} \int_{0}^{1}\left|f+\sqrt{u} P^{N} Y\right|^{2} d t \\
& +\frac{1}{2} \int_{0}^{1}|f|^{2} d t-\frac{\sqrt{u}}{2}\left(\left|P^{N} Y_{1}\right|^{2}-\left|P^{N} Y_{0}\right|^{2}-\operatorname{dim} N_{R}\right) .
\end{aligned}
$$

From (7.26) and (7.27), we get

$$
\begin{aligned}
& I=\exp \left\{\frac{1}{2} \int_{0}^{1}|f|^{2} d t+\frac{\sqrt{u}}{2} \operatorname{dim} N_{R}\right\} \\
& \times \int_{N_{R}} E^{Q_{(Y, Y)}}\left[\operatorname { e x p } \left\{\frac{1}{2} \int_{0}^{1}\left\langle f+\sqrt{u} P^{N} Y, \delta Y\right\rangle\right.\right. \\
& \left.\left.\quad-\frac{1}{2} \int_{0}^{1}\left|f+\sqrt{u} P^{N} Y\right|^{2} d t\right\}\right] \frac{d v_{N}(Y)}{(2 \pi)^{\operatorname{dim} N}} .
\end{aligned}
$$

Let $w$ be a standard Brownian motion in $M_{R}$, with $w_{0}=0$. Given $Y \in N_{R}$, consider the stochastic differential equation

$$
\begin{gathered}
d Y^{\prime}=\left(\sqrt{u} P^{N} Y^{\prime}+f\right) d t+d w, \\
Y_{0}^{\prime}=Y .
\end{gathered}
$$

Then by using the properties of the Girsanov transformation [IkW, p. 178; B6, $\S 3]$, we know that

$$
E^{Q_{(Y, Y)}}\left[\exp \left\{\int_{0}^{1}\left\langle f+\sqrt{u} P^{N} Y, \delta Y\right\rangle-\frac{1}{2} \int_{0}^{1}\left|f+\sqrt{u} P^{N} Y\right|^{2} d t\right\}\right]
$$

is exactly the value at $Y$ of the density of the probability law of $Y_{1}^{\prime}$ in (7.29) with respect to the measure $d v_{M}(Y) /(2 \pi)^{\operatorname{dim} M}$.

Now (7.29) can be explicitly integrated by the formula

$$
\begin{aligned}
Y_{t}^{\prime}= & P^{L}\left(\int_{0}^{t} f d s+w_{t}\right) \\
& +P^{N}\left(e^{\sqrt{u} t} Y+e^{\sqrt{u} t} \int_{0}^{t} e^{-\sqrt{u} s} f_{s} d s+e^{\sqrt{u} t} \int_{0}^{t} e^{-\sqrt{u} s} d w_{s}\right) .
\end{aligned}
$$

From (7.31), we deduce that $P^{L} Y_{1}^{\prime}$ and $P^{N} Y_{1}$ are independent and have Gaussian laws, respectively given by

$$
\exp \left\{-\frac{1}{2}\left|Y^{\prime}-\int_{0}^{1} P^{L} f d s\right|^{2}\right\} \frac{d v_{L}\left(Y^{\prime}\right)}{(2 \pi)^{\operatorname{dim} L}}
$$




$$
\begin{aligned}
\left(\frac{2 \sqrt{u}}{e^{2 \sqrt{u}}-1}\right)^{\operatorname{dim} N} \exp \{- & \frac{1}{2}\left(\frac{2 \sqrt{u}}{e^{2 \sqrt{u}}-1}\right) \\
& \left.\times\left|Y^{\prime \prime}-e^{\sqrt{u}} Y-e^{\sqrt{u}} \int_{0}^{1} e^{-\sqrt{u} s} f d s\right|^{2}\right\} \frac{d v_{N}\left(Y^{\prime \prime}\right)}{(2 \pi)^{\operatorname{dim} N}} .
\end{aligned}
$$

From (7.28) and (7.32), we get

$$
I=\exp \left\{\int_{0}^{1} \frac{|f|^{2} d s}{2}-\frac{1}{2}\left|\int_{0}^{1} P^{L} f d s\right|^{2}\right\} \frac{1}{(2 \sinh (\sqrt{u} / 2))^{\operatorname{dim} N_{R}}} .
$$

From (7.26) and (7.33), we find that

$$
\begin{aligned}
& \int_{N_{R}} E^{Q_{(Y, Y)}}\left[\exp \left\{\int_{0}^{1}\left\langle f, d Y-\sqrt{u} P^{N} Y d t\right\rangle-\frac{u}{2} \int_{0}^{1}\left|P^{N} Y\right|^{2} d t\right\}\right] \frac{d v_{N}(Y)}{(2 \pi)^{\operatorname{dim} N}} \\
& \quad=\frac{\exp \left\{\frac{1}{2} \int_{0}^{1}|f|^{2} d t-\frac{1}{2}\left|\int_{0}^{1} P^{L} f d t\right|^{2}\right\}}{u^{\operatorname{dim} N} \operatorname{det}^{1 / 2}\left(I_{H_{N}}-u P^{N} J^{-2}\right)} .
\end{aligned}
$$

Let $P_{1}$ be the probability law on $\mathscr{C}\left([0,1] ; L_{R}\right)$ of the Brownian bridge $Y^{L}$, with $Y_{0}^{L}=Y_{1}^{L}=0$. Let $P_{2}$ be the probability law on $\mathscr{C}\left(S_{1} ; N_{R}\right)$ of the Gaussian process $Y^{N}$ whose covariance is given by the operator

$$
C_{u}=\left(-J^{2}+u\right)^{-1} \text { on } S_{1}
$$

(i.e., $C_{u}=(-\Delta+u)^{-1}$ on $[0,1]$ with periodic boundary conditions). From (7.34), we deduce the identity of positive measures on $\mathscr{C}\left([0,1] ; M_{R}\right)$ :

$$
\begin{gathered}
\int_{N_{R}} \exp \left\{-\frac{u}{2} \int_{0}^{1}\left|P^{N} Y\right|^{2} d t\right\} d Q_{(Y, Y)} \frac{d v_{N}(Y)}{(2 \pi)^{\operatorname{dim} N}} \\
=\frac{d P_{1}\left(Y^{L}\right) d P_{2}\left(Y^{N}\right)}{u^{\operatorname{dim} N} \operatorname{det}^{1 / 2}\left(I_{H_{N}}-u P^{N} J^{-2}\right)}
\end{gathered}
$$

From (7.25) and (7.36), we get

$$
\begin{aligned}
\operatorname{Tr}_{s}\left[\exp \left(-\mathscr{B}_{u}^{2}+b N_{H}\right)\right] \\
=\exp \left\{-\frac{1}{2} \operatorname{Tr}\left[R^{L}\right]+\frac{b}{2} \operatorname{dim} N\right\} \frac{\Psi\left(u, R^{N}+b I_{N}^{\prime}\right)}{\operatorname{det}^{1 / 2}\left(I_{H_{N}}-u P^{N} J^{-2}\right)} \\
\times E^{P_{1} \otimes P_{2}}\left[\operatorname { e x p } \left\{\frac{1}{2} \int_{0}^{1}\left\langle R^{M} Y, d Y\right\rangle\right.\right. \\
\left.\left.\quad-\frac{u}{2}\left\langle P^{L} A\left(J^{2}-\left(R^{N}+b I_{N}^{\prime}\right) J-u I_{H_{N}}\right)^{-1} J A P^{L} Y, Y\right\rangle\right\}\right] .
\end{aligned}
$$

Let $P_{1}^{\prime}$ be the image of $P_{1}$ by the map

$$
Y^{L} \in \mathscr{C}\left([0,1] ; L_{R}\right) \rightarrow Y^{L}-\int_{0}^{1} Y^{L} d s \in \mathscr{C}\left([0,1] ; L_{R}\right) \cap H_{L} .
$$


Observe that $\int_{0}^{1}\left\langle R^{M} Y, d Y\right\rangle$ and $\left\langle P^{L} A\left(J^{2}-\left(R^{N}+b I_{N}^{\prime}\right) J-u I_{H_{N}}\right)^{-1} J A P^{L} Y, Y\right\rangle$ are invariant under the maps $Y \in \mathscr{C}\left([0,1] ; M_{R}\right) \rightarrow Y+a \in \mathscr{C}\left([0,1] ; M_{R}\right)$ (with $a \in M_{R}$ ). Therefore in (7.37), we may and we will replace $E^{P_{1} \otimes P_{2}}$ by $E^{P_{1}^{\prime} \otimes P_{2}}$.

Let $(\Omega, \mathscr{A}, P)$ be a probability space. Let $\left(Y_{n}, Z_{n}\right)_{n \in \mathbb{N}^{*}}$ and let $X_{0}^{\prime},\left(Y_{n}^{\prime}, Z_{n}^{\prime}\right)_{n \in \mathbb{N}^{*}}$ be independent Gaussian random variables with values respectively in $L_{R}$ and $N_{R}$, which have mean zero and covariance $I_{L_{R}}$ and $I_{N_{R}}$ respectively. Set

$$
\begin{gathered}
Y_{t}^{L}=\sqrt{2} \sum_{n=1}^{+\infty}\left(\frac{Y_{n}}{2 \pi n} \sin (2 \pi n t)+\frac{Z_{n}}{2 \pi n} \cos (2 \pi n t)\right), \\
Y_{t}^{N}=\frac{X_{0}^{\prime}}{\sqrt{u}}+\sqrt{2} \sum_{n=1}^{+\infty}\left(\frac{Y_{n}^{\prime}}{\sqrt{4 \pi^{2} n^{2}+u}} \sin (2 \pi n t)+\frac{Z_{n}^{\prime}}{\sqrt{4 \pi^{2} n^{2}+u}} \cos (2 \pi n t)\right) .
\end{gathered}
$$

Then by [IN, Theorems 4.1 and 5.1], both series converge $P$-uniformly on $[0,1]$. Moreover $Y_{t}=\left(Y_{t}^{L}, Y_{t}^{N}\right)$ is a Gaussian process. Inspection of the covariance shows that under $P$, the probability law of $Y$ is exactly $P_{1}^{\prime} \otimes P_{2}$. Set

$$
U_{n}=\left(\frac{Y_{n}}{2 \pi n}, \frac{Y_{n}^{\prime}}{\sqrt{4 \pi^{2} n^{2}+u}}\right), \quad V_{n}=\left(\frac{Z_{n}}{2 \pi n}, \frac{Z_{n}^{\prime}}{\sqrt{4 \pi^{2} n^{2}+u}}\right) .
$$

Then by proceeding as in [B10, proof of Theorem 5.3], we find easily that

$$
\int_{0}^{1}\left\langle R^{M} Y, d Y\right\rangle=-\sum_{1}^{+\infty} 4 \pi n\left\langle R^{M} U_{n}, V_{n}\right\rangle
$$

and the series in the right-hand side of (7.40) is a martingale which converges $P$ almost surely and $L_{2}(\Omega, P)$. Using the fact that if $X$ is a real random variable with Gaussian distribution, then $E\left[\exp \left(\alpha X^{2} / 2\right)\right]=(1-\alpha)^{-1 / 2}$ for $0<\alpha<1$, and [DeM; p. 28] one finds easily that the convergence in the right-hand side of (7.40) takes place in all the $L_{p}(\Omega, P) \quad(1 \leq p<+\infty)$.

Also the operator $\left(J^{2}-\left(R^{N}+b I_{N}^{\prime}\right) J-u I_{H_{N}}\right)^{-1} J$ is a continuous operator acting on $L_{2}\left([0,1] ; M_{R}\right)$. Since $(7.38)$ is exactly the Fourier expansion of the process $Y_{t} \in L_{2}\left([0,1] ; M_{R}\right)$, we find that

$$
-\frac{u}{2}\left\langle P^{L} A\left(J^{2}-\left(R^{N}+b I_{N}^{\prime}\right) J-u I_{H_{N}}\right)^{-1} J A P^{L} Y, Y\right\rangle
$$

can also be expressed as the sum of an infinite series of terms which are quadratic in $\left(Y_{n}, Z_{n}\right)$ and which converges $P$-almost surely and in all the $L_{p}(\Omega, P)$ $(1 \leq p<+\infty)$. Neither this series nor the series in (7.40) contains $X_{0}^{\prime}$.

Set

$$
K=\int_{0}^{1}\left\langle R^{M} Y, d Y\right\rangle-u\left\langle P^{L} A\left(J^{2}-\left(R^{N}+b I_{N}^{\prime}\right) J-u I_{H_{N}}\right)^{-1} J A P^{L} Y, Y\right\rangle .
$$


By the previous considerations, $K$ is the sum of a series of terms which are quadratic in $\left(U_{n}, V_{n}\right)$ and which converges $P$-almost surely and in all the $L_{p}(\Omega, P) \quad(1 \leq p<+\infty)$. Let $K_{n}$ be the sum of the first $n$ terms in the series. We claim that

$$
\lim _{n \rightarrow+\infty} E^{P_{1}^{\prime} \otimes P_{2}}\left[\exp \left(\frac{K_{n}}{2}\right)\right]=E^{P_{1}^{\prime} \otimes P_{2}}\left[\exp \left(\frac{K}{2}\right)\right] .
$$

In fact since $K_{n}$ is of positive degree in the Grassmann variables of $\Lambda\left(T_{R}^{*} B\right)$, the expansion

$$
\exp \left(\frac{K_{n}}{2}\right)=1+\frac{K_{n}}{2}+\cdots
$$

terminates after a finite number of terms. Using the fact that $K_{n} \rightarrow K \quad P$-almost surely and also uniform integrability, we obtain (7.42).

Now $K_{n}$ is a finite sum of independent random variables which are quadratic in $\left(U_{k}, V_{k}\right)(k \leq n)$. One then easily verifies that

$$
\begin{aligned}
& \frac{1}{\left[\prod_{k=1}^{n}\left(1+u / 4 \pi^{2} k^{2}\right)\right]^{\operatorname{dim} N_{R}}} E^{P_{1}^{\prime} \otimes P_{2}}\left[\exp \left(\frac{K_{n}}{2}\right)\right] \\
& =\left[\prod _ { \substack { k = - n \\
k \neq 0 } } ^ { + n } \operatorname { d e t } ^ { 1 / 2 } \left(I_{M_{R}}-R^{M}(2 \pi i k)^{-1}-u P^{L} A\left(-4 \pi^{2} k^{2}-\left(R^{N}+b I_{N}^{\prime}\right) 2 i \pi k-u I_{N_{R}}\right)^{-1}\right.\right. \\
& \left.\left.\times(2 i \pi k)^{-1} A P^{L}+u P^{N}\left(4 \pi^{2} k^{2}\right)^{-1}\right)\right]^{-1} .
\end{aligned}
$$

From (7.42) and (7.44), we get

$$
\begin{aligned}
& \frac{1}{\operatorname{det}^{1 / 2}\left(I-u P^{N} J^{-2}\right)} E^{P_{1}^{\prime} \otimes P_{2}}\left[\exp \left(\frac{K}{2}\right)\right] \\
&=\left[\operatorname { d e t } ^ { 1 / 2 } \left(I_{H_{M}}-R^{M} J^{-1}-u P^{L} A\left(J^{2}-\left(R^{N}+b I_{N}^{\prime}\right) J-u I_{H_{N}}\right)^{-1} A P^{L} J^{-1}\right.\right. \\
&\left.\left.-u P^{N} J^{-2}\right)\right]^{-1} .
\end{aligned}
$$

Our theorem now follows from (7.23), (7.37), and (7.45).

Remark 7.4. Theorem 4.8 is now an obvious consequence of Theorem 7.3. In fact using (7.24), one easily verifies that as $u \rightarrow 0$

$$
\begin{aligned}
\operatorname{Tr}_{s}\left[\exp \left(-\mathscr{B}_{u}^{2}+b N_{H}\right)\right]= & \exp \left\{-\frac{1}{2} \operatorname{Tr}\left[R^{L}\right]+\frac{b}{2} \operatorname{dim} N\right\} \\
& \times \frac{\operatorname{det}^{1 / 2}\left(I_{H_{N}}-\left(R^{N}+b I_{N}^{\prime}\right) J^{-1}\right)}{\operatorname{det}^{1 / 2}\left(I_{H_{M}}-R^{M} J^{-1}\right)}+O(u) .
\end{aligned}
$$


Now if $C \in$ End $N$ is skew-adjoint and has eigenvalues $x_{1}, \ldots, x_{n}$, then by (6.16) and (6.17)

$$
\operatorname{det}^{1 / 2}\left(I_{H_{N}}-C J^{-1}\right)=\prod_{1}^{n} \frac{\sinh \left(x_{j} / 2\right)}{x_{j} / 2} .
$$

From (7.15), (7.46), and (7.47), we find that as $u \rightarrow 0$,

$$
\operatorname{Tr}_{s}\left[\exp \left(-\mathscr{B}_{u}^{2}+b N_{H}\right)\right]=\frac{\operatorname{Td}\left(-R^{M}\right)}{\operatorname{Td}\left(-R^{N}-b I_{N}\right)}+O(u),
$$

which is equivalent to (4.71). One verifies easily (by using, say, the analyticity of the considered expressions in the variable b) that the derivatives in $b$ verify the obvious analogue of (7.48). The second line of (4.72) is proved as well.

Remark 7.5. The results of Theorem 4.6 are not obvious consequences of the explicit formulas of Theorem 7.3. In $\S 9$, we will give a direct proof that the expression in the right-hand side of (7.24) verifies the equations of Theorem 4.6 by using infinite-dimensional intersection theory.

(c) The asymptotics of the generalized supertraces as $u \rightarrow+\infty$. Recall that by (6.1) and (6.17)

$$
\operatorname{det}^{1 / 2}\left(I_{H_{N}}-u P^{N} J^{-2}\right)=\left(\frac{\sinh (\sqrt{u} / 2)}{\sqrt{u} / 2}\right)^{\operatorname{dim} N_{R}}
$$

We now use the same notation as in $\S 4(f)$. Namely if $\omega_{u}(b)$ and $\omega_{\infty}(b)$ are smooth differential forms on $B$ depending smoothly on $b \in \mathbb{C}$, we will write that as $u \rightarrow+\infty$

$$
\omega_{u}(b)=\omega_{\infty}(b)+O(1 / \sqrt{u})
$$

if for any $k \in \mathbb{N}$, the sup of the derivatives of order $\leq k$ of $\omega_{u}(b)-\omega_{\infty}(b)$ in the variables $(x, b) \in B \times[b \in \mathbb{C} ;|b| \leq 1\}$ is dominated by $C_{k} / \sqrt{u}$ for $u \geq 1$.

Theorem 7.6. As $u \rightarrow+\infty$

$$
\begin{aligned}
& \operatorname{det}\left(I_{H_{N}}-\left(R^{N}+b I_{N}^{\prime}\right) J^{-1}-u J^{-2}\right)=\operatorname{det}\left(I_{H_{N}}-u J^{-2}\right)\left(1+O\left(\frac{1}{\sqrt{u}}\right)\right), \\
& \operatorname{det}^{1 / 2}\left(I_{H_{M}}-R^{M} J^{-1}-u P^{L} A\left(J^{2}-\left(R^{N}+b I_{N}^{\prime}\right) J-u I_{H_{N}}\right)^{-1} A P^{L} J^{-1}-u P^{N} J^{-2}\right) \\
& \quad=\operatorname{det}^{1 / 2}\left(I_{H_{N}}-u J^{-2}\right) \operatorname{det}^{1 / 2}\left(I_{H_{L}}-R^{L} J^{-1}\right)(1+O(1 / \sqrt{u})) .
\end{aligned}
$$

Proof. Clearly the first equation of (7.51) is a special case of the second. Still we give a separate proof of the first equation. By (7.23)

$$
\operatorname{det}^{1 / 2}\left(I_{H_{N}}-\left(R^{N}+b I_{N}^{\prime}\right) J^{-1}-u J^{-2}\right)=\Psi\left(u, R^{N}+b I_{N}^{\prime}\right) .
$$

Also if $x \in \mathbb{C}$, as $u \rightarrow+\infty$

$$
\frac{2}{u} \operatorname{ch}\left(\frac{\sqrt{x^{2}+4 u}}{2}\right)=\frac{e^{\sqrt{u}}}{u}\left(1+O\left(\frac{1}{\sqrt{u}}\right)\right) .
$$


The first equation in (7.51) trivially follows from (6.2), (6.15), (7.49), (7.52), and (7.53).

We now prove the second equation in (7.51). Remember that if $C$ is a square matrix written in the form

$$
C=\left(\begin{array}{ll}
E & F \\
G & H
\end{array}\right)
$$

if $E$ is a square matrix which is invertible, then

$$
\operatorname{det} C=\operatorname{det}(E) \operatorname{det}\left(H-G E^{-1} F\right) \text {. }
$$

By (7.19), we may express

$D=\operatorname{det}\left(I_{H_{M}}-R^{M} J^{-1}-u P^{L} A\left(J^{2}-\left(R^{N}+b I_{N}^{\prime}\right) J-u I_{H_{N}}\right)^{-1} A P^{L} J^{-1}-u P^{N} J^{-2}\right)$

as an infinite product of finite-dimensional determinants of linear operators in End $M_{R}$. Now $M_{R}=L_{R} \oplus N_{R}$. We can then use formula (7.55) on each of these finite-dimensional determinants. We get

$$
\begin{aligned}
D=\operatorname{det}\left(I_{H_{N}}-u J^{-2}-P^{N} R^{M} P^{N} J^{-1}\right) \\
\times \operatorname{det}\left(I_{H_{L}}-P^{L} R^{M} P^{L} J^{-1}-u P^{L} A\left(J^{2}-\left(R^{N}+b I_{N}^{\prime}\right) J-u I_{H_{N}}\right)^{-1} J^{-1} A P^{L}\right. \\
\left.\quad-P^{L} R^{M} P^{N}\left(I_{H_{N}}-u P^{N} J^{-2}-P^{N} R^{M} P^{N} J^{-1}\right)^{-1} P^{N} R^{M} P^{L} J^{-2}\right) .
\end{aligned}
$$

Of course, the determinants in the right-hand side of (7.57) are normalized determinants in the sense of $\S 7$. By the same argument as in (7.53) and (7.54), as $u \rightarrow+\infty$

$$
\operatorname{det}\left(I_{H_{N}}-u J^{-2}-P^{N} R^{M} P^{N} J^{-1}\right)=\operatorname{det}\left(I_{H_{N}}-u J^{-2}\right)\left(1+O\left(\frac{1}{\sqrt{u}}\right)\right)
$$

We now use the straightforward identity

$$
\begin{aligned}
& -u P^{L} A\left(J^{2}-\left(R^{N}+b I_{N}^{\prime}\right) J-u I_{H_{N}}\right)^{-1} A P^{L} J^{-1} \\
& \quad=P^{L} A^{2} P^{L} J^{-1}-P^{L} A\left(J-R^{N}-b I_{N}^{\prime}\right)\left(J^{2}-\left(R^{N}+b I_{N}^{\prime}\right) J-u I_{H_{N}}\right)^{-1} A P^{L} .
\end{aligned}
$$

Set

$$
\begin{gathered}
K_{u}=\left(J-R^{N}-b I_{N}^{\prime}\right)\left(J^{2}-\left(R^{N}+b I_{N}^{\prime}\right) J-u I_{H_{N}}\right)^{-1}, \\
L_{u}=I_{H_{N}}-u P^{N} J^{-2}-P^{N} R^{M} P^{N} J^{-1}, \\
\alpha=P^{L}\left(R^{M}-A^{2}\right) P^{L} J^{-1} \\
\beta=P^{L} A K_{u} A P^{L}+P^{L} R^{M} P^{N} L_{u}^{-1} P^{N} R^{M} P^{L} J^{-2} .
\end{gathered}
$$

For $t \in[0,1]$, we claim that

$$
\frac{\partial}{\partial t} \log \left(\operatorname{det}\left(I_{H_{L}}-\alpha-t \beta\right)\right)=-\operatorname{Tr}\left[\left(I_{H_{L}}-\alpha-t \beta\right)^{-1} \beta\right] .
$$


(7.61) can easily be derived by expressing $\operatorname{det}\left(I_{H_{L}}-\alpha-t \beta\right)^{-1}$ as a convergent product of finite-dimensional determinants and by using formula (7.61) in a finite-dimensional situation. Of course in (7.61) $\operatorname{Tr}\left[\left(I_{H_{L}}-\alpha-t \beta\right)^{-1} \beta\right]$ is also a regularized trace in the sense of (7.17). Clearly,

$$
\operatorname{Tr}\left[\left(I_{H_{L}}-\alpha-t \beta\right)^{-1} \beta\right]=\operatorname{Tr}[\beta]+\operatorname{Tr}\left[\left(I_{H_{M}}-\alpha-t \beta\right)^{-1}(\alpha+t \beta) \beta\right] .
$$

We first estimate $\operatorname{Tr}[\beta]$. We have the inequality

$$
\begin{aligned}
& \left|\operatorname{Tr}\left[P^{L} R^{M} P^{N} L_{u}^{-1} P^{N} R^{M} P^{L} J^{-2}\right]\right| \\
& \quad \leq C \sum_{1}^{+\infty} \frac{1}{k^{2}+u} \leq C \int_{0}^{+\infty} \frac{d x}{x^{2}+u}=\frac{C \pi}{2 \sqrt{u}} .
\end{aligned}
$$

Similarly,

$$
\begin{aligned}
& \left|\operatorname{Tr}\left[P^{L} A\left(R^{N}+b I_{N}^{\prime}\right)\left(J^{2}-\left(R^{N}+b I_{N}^{\prime}\right) J-u I_{H_{N}}\right)^{-1} A P^{L}\right]\right| \\
& \quad \leq C \sum_{1}^{+\infty} \frac{1}{k^{2}+u} \leq \frac{C \pi}{2 \sqrt{u}} .
\end{aligned}
$$

Also for $x \in \mathbb{C},|x| \leq 1, k \in \mathbb{N}^{*}$, and $u \geq 0$

$$
\begin{aligned}
\frac{2 \pi i k}{-4 \pi^{2} k^{2}-2 \pi i k x-u}-\frac{2 \pi i k}{-4 \pi^{2} k^{2}+2 \pi i k x-u} \\
=\frac{-8 \pi^{2} k^{2} x}{\left(-4 \pi^{2} k^{2}-2 \pi i k x-u\right)\left(-4 \pi^{2} k^{2}+2 \pi i k x-u\right)} .
\end{aligned}
$$

Therefore for $x \in \mathbb{C},|x| \leq 1$, and $u \geq 1$

$$
\left|\frac{2 \pi i k}{-4 \pi^{2} k^{2}-2 \pi i k x-u}-\frac{2 \pi i k}{-4 \pi^{2} k^{2}+2 \pi i k x-u}\right| \leq \frac{C k^{2}}{k^{4}+u^{2}} .
$$

From (7.66), we deduce that for $u \geq 1$

$$
\begin{aligned}
& \left|\operatorname{Tr}\left[P^{L} A J\left(-J^{2}-\left(R^{N}+b I_{N}^{\prime}\right) J-u I_{H_{N}}\right)^{-1} A P^{L}\right]\right| \\
& \quad \leq C \sum_{1}^{+\infty} \frac{k^{2}}{k^{4}+u^{2}} \leq 2 C \int_{0}^{+\infty} \frac{x^{2} d x}{x^{4}+u^{2}}=\frac{2 C}{\sqrt{u}} \int_{0}^{+\infty} \frac{x^{2} d x}{x^{4}+1} .
\end{aligned}
$$

From (7.63)-(7.67), we find that for $u \geq 1$

$$
|\operatorname{Tr}[\beta]| \leq \frac{C}{\sqrt{u}} .
$$

We now estimate $\operatorname{Tr}\left[\left(I_{H_{L}}-\alpha-t \beta\right)^{-1}(\alpha+t \beta) \beta\right]$. Using (7.63), one verifies easily that for $t \in[0,1]$ and $u \geq 1$

$$
\begin{aligned}
& \left|\operatorname{Tr}\left[\left(I_{H_{L}}-\alpha-t \beta\right)^{-1}(\alpha+t \beta) \beta\right]\right| \\
& \quad \leq C\left\{\sum_{1}^{+\infty}\left(\frac{1}{k}\left(\frac{k}{k^{2}+u}\right)+\frac{k^{2}}{k^{4}+u^{2}}\right)\right\} .
\end{aligned}
$$


By using the same argument as in (7.63)-(7.68), we deduce from (7.69) that for $t \in[0,1]$ and $u \geq 1$

$$
\left|\operatorname{Tr}\left[\left(I_{H_{L}}-\alpha-t \beta\right)^{-1}(\alpha+t \beta) \beta\right]\right| \leq \frac{C}{\sqrt{u}} .
$$

From (7.61), (7.68), and (7.70), we get for $t \in[0,1]$ and $u \geq 1$

$$
\left|\frac{\partial}{\partial t} \log \left[\operatorname{det}\left(I_{H_{L}}-\alpha-t \beta\right)\right]\right| \leq \frac{C}{\sqrt{u}} .
$$

From (7.71), we find that

$$
\left|\log \operatorname{det}\left(I_{H_{L}}-\alpha-\beta\right)-\log \operatorname{det}\left(I_{H_{L}}-\alpha\right)\right| \leq \frac{C}{\sqrt{u}} .
$$

From (7.57)-(7.59) and (7.72), and from the fact that the restriction of $P^{L}\left(R^{M}-A^{2}\right) P^{L}$ to $L$ coincides with $R^{L}$, we get the second equation in (7.52). Our theorem in proved.

We finally obtain the key result.

Theorem 7.7. As $u \rightarrow+\infty$

$$
\operatorname{Tr}_{s}\left[\exp \left(-\mathscr{B}_{u}^{2}+b N_{H}\right)\right]=\exp \left(\frac{b}{2} \operatorname{dim} N\right) \operatorname{Td}\left(-R^{L}\right)+O\left(\frac{1}{\sqrt{u}}\right) .
$$

In particular, as $u \rightarrow+\infty$

$$
\begin{gathered}
\operatorname{Tr}_{s}\left[\exp \left(-\mathscr{B}_{u}^{2}\right)\right]=\operatorname{Td}\left(-R^{L}\right)+O\left(\frac{1}{\sqrt{u}}\right), \\
\frac{\partial}{\partial b} \operatorname{Tr}_{s}\left[\exp \left(-\mathscr{B}_{u}^{2}+b N_{H}\right)\right]_{b=0}=\frac{\operatorname{dim} N}{2} \operatorname{Td}\left(-R^{L}\right)+O\left(\frac{1}{\sqrt{u}}\right) .
\end{gathered}
$$

Proof. By Theorems 7.3 and 7.6, we find that as $u \rightarrow+\infty$

$$
\operatorname{Tr}_{s}\left[\exp \left(-\mathscr{B}_{u}^{2}+b N_{H}\right)\right]=\frac{\exp \left\{-\frac{1}{2} \operatorname{Tr}\left(R^{L}\right)+\frac{b}{2} \operatorname{dim} N\right\}}{\operatorname{det}^{1 / 2}\left(I_{H_{L}}-R^{L} J^{-1}\right)}+O\left(\frac{1}{\sqrt{u}}\right) .
$$

By the same arguments as in (7.46)-(7.48), we find that (7.75) is equivalent to (7.73). (7.74) immediately follows.

Remark 7.8. From (7.74), we find in particular that as $u \rightarrow \infty$

$$
\frac{\partial}{\partial b} \operatorname{Tr}_{s}\left[\exp \left(-\mathscr{B}_{u}^{2}+b\left(N_{H}-\frac{\operatorname{dim} N}{2}\right)\right)\right]_{b=0}=O\left(\frac{1}{\sqrt{u}}\right) .
$$

From many points of view, the choice of $N_{H}-(\operatorname{dim} N) / 2$ instead of $N_{H}$ is more natural in the double transgression formulas of Theorem 4.6. We will give a (hopefully) transparent explanation for (7.73), (7.74), and (7.76) in $\S 9$. Also (7.76) is directly connected with Theorem 1.6 which asserts in particular that

$$
\left\langle\left(N_{H}-\frac{\operatorname{dim} N}{2}\right) \beta, \beta\right\rangle=0 \text {. }
$$


In fact by rescaling the variables in $N_{R}$, the operator $D^{N}+\sqrt{u} V\left(P^{N} Y\right)$ is changed into $u^{1 / 4}\left(D^{N}+V\left(P^{N} Y\right)\right)$, and as $u \rightarrow+\infty$ only the kernel of $D^{N}+$ $V\left(P^{N} Y\right)$ should be relevant in the evaluation of the limit of $\operatorname{Tr}_{s}\left[\exp \left(-\mathscr{B}_{\mu}^{2}+\right.\right.$ $\left.b N_{H}\right)$ ]. This roughly accounts for the fact that $N_{H}$ is changed into $(\operatorname{dim} N) / 2$ when $u \rightarrow+\infty$.

Theorem 2.7 , and more precisely equation (2.24), explains partly why the curvature $R^{L}$ of the connection $\nabla^{L}$ appears in the limit as $u \rightarrow+\infty$ of the forms $\operatorname{Tr}_{s}\left[\exp \left(-\mathscr{B}_{u}^{2}+b N_{H}\right)\right]$. In Bismut-Lebeau [BL2, §14], such an argument is fully exploited to give another proof of Theorem 7.7.

Remark 7.9. From Theorems 4.8 and 7.7, we find that the family of smooth forms $\operatorname{Tr}_{s}\left[\exp \left(-\mathscr{B}_{u}^{2}\right)\right]$ interpolates between the forms $\operatorname{Td}\left(-R^{M}\right) / \operatorname{Td}\left(-R^{N}\right)$ (for $u=0$ ) and $\operatorname{Td}\left(-R^{L}\right)$ (for $u=+\infty$ ). This fact will be systematically exploited in the sequel.

\section{Generalized supertraces, ANALytic torsion, AND the TOdD GeNus}

The purpose of this section is to construct generalized analytic torsion forms associated with the acyclic chain complex of holomorphic Hermitian vector bundles

$$
0 \rightarrow L \underset{i}{\rightarrow} M \underset{j}{\rightarrow} N \rightarrow 0 .
$$

This is done by integrating the double transgression formulas of Theorem 4.6 by a zeta function technique. If $g^{M}$ is the metric on $M$, we thus obtain a form $\mathbb{B}\left(L, M, g^{M}\right)$ in $P^{B}$.

The main result of this paper is our evaluation of $\mathbb{B}\left(L, M, g^{M}\right) \in P^{B} / P^{B, 0}$ as the sum of a standard Bott-Chern class [BoC, D, BGS1], and of an additive class naturally associated with the derivative at 0 of the Mellin transform of $(-(\partial \varphi / \partial x) / \varphi)(u, x)$ evaluated on $N$.

The explicit evaluation of this Mellin transform is done in a joint appendix with C. Soulé. The main result of the appendix is that this additive class coincides (up to a rather irrelevant term) with a class introduced by Gillet and Soulé [GS].

This section is organized as follows. In (a), we construct the generalized analytic torsion forms $\mathbb{B}\left(L, M, g^{M}\right)$, and evaluate $\frac{\bar{\partial} \partial}{2 i \pi} \mathbb{B}\left(L, M, g^{M}\right)$. In (b), we calculate $\mathbb{B}\left(L, M, g^{M}\right)$ modulo $\partial$ and $\bar{\partial}$ coboundaries.

Our assumptions and notations are the same as in $\S \S 3,4,6$, and 7.

(a) A construction of the generalized analytic torsion forms. We now imitate a construction of Bismut-Gillet-Soulé [BGS1, Definition 1.16], [BGS4, Definition $2.1]$ to integrate the double transgression formulas of Theorem 4.6 by a zeta function technique. 
Definition 8.1. For $s \in \mathbb{C}, 0<\operatorname{Re}(s)<\frac{1}{2}$, let $A(s)$ be the following smooth even form on $B$ :

$$
\begin{aligned}
A(s)=\frac{1}{\Gamma(s)} \int_{0}^{+\infty} u^{s-1}\left\{\frac { \partial } { \partial b } \left[\operatorname{Tr}_{s}[\right.\right. & \left.\left.\exp \left(-\mathscr{B}_{u}^{2}+b N_{H}\right)\right]\right]_{b=0} \\
& \left.-\frac{\operatorname{dim} N}{2} \operatorname{Td}\left(-R^{L}\right)\right\} d u .
\end{aligned}
$$

Note that by Theorems 4.8 and 7.7, the integral in the right-hand side of (8.1) defines indeed a smooth form. More precisely we find that the map $s \in \mathbb{C}$, $0<\operatorname{Re}(s)<\frac{1}{2} \rightarrow A(s)$, extends into a meromorphic function of $s$ which is holomorphic near $s=0$.

In particular, by using Theorem 4.8, we find that the derivative $A^{\prime}(0)$ is given by the formula

$$
\begin{aligned}
A^{\prime}(0)= & \int_{0}^{1}\left\{\frac{\partial}{\partial b}\left[\operatorname{Tr}_{s}\left[\exp \left(-\mathscr{B}_{u}^{2}+b N_{H}\right)\right]\right]_{b=0}\right. \\
& \left.+\operatorname{Td}\left(-R^{M}\right)\left(\operatorname{Td}^{-1}\right)^{\prime}\left(-R^{N}\right)\right\} \frac{d u}{u} \\
& +\int_{1}^{+\infty}\left\{\frac{\partial}{\partial b}\left[\operatorname{Tr}_{s}\left[\exp \left(-\mathscr{B}_{u}^{2}+b N_{H}\right)\right]\right]_{b=0}-\frac{\operatorname{dim} N}{2} \operatorname{Td}\left(-R^{L}\right)\right\} \frac{d u}{u} \\
& +\Gamma^{\prime}(1)\left\{\operatorname{Td}\left(-R^{M}\right)\left(\operatorname{Td}^{-1}\right)^{\prime}\left(-R^{N}\right)+\frac{\operatorname{dim} N}{2} \operatorname{Td}\left(-R^{L}\right)\right\} .
\end{aligned}
$$

Note that the form which appears in (8.2) after $\Gamma^{\prime}(1)$,

$$
\operatorname{Td}\left(-R^{M}\right)\left(\mathrm{Td}^{-1}\right)^{\prime}\left(-R^{N}\right)+\frac{\operatorname{dim} N}{2} \operatorname{Td}\left(-R^{L}\right),
$$

is closed. This is a phenomenon which was already observed in a finite-dimensional context in Bismut-Gillet-Soule [BGS4, §2].

Also by Theorem 4.6 , we can replace $\frac{\partial}{\partial b}\left[\operatorname{Tr}_{s}\left[\exp \left(-\mathscr{B}_{u}^{2}+b N_{H}\right)\right]\right]_{b=0}$ by $\operatorname{Tr}_{s}\left[N_{H} \exp \left(-\mathscr{B}_{u}^{2}\right)\right]$ in (8.1) and (8.2). This makes formulas (8.1) and (8.2) very similar to [BGS4, (2.1), (2.3)].

Let $\Phi$ be the linear map from $\Lambda^{\text {even }}\left(T_{R}^{*} B\right)$ into itself which associates $(2 \pi i)^{-q} \omega$ tc $\omega \in \Lambda^{2 q}\left(T_{R}^{*} B\right)$.

Definition 8.2. Let $\mathbb{B}\left(L, M, g^{M}\right)$ be the smooth form on $B$ :

$$
\mathbb{B}\left(L, M, g^{M}\right)=\Phi\left(A^{\prime}(0)\right) \text {. }
$$

The notation $\mathbb{B}\left(L, M, g^{M}\right)$ can be easily explained. In fact, the embedding $0 \rightarrow L \rightarrow M$ determines $N$. Also the metric $g^{M}$ induces the metrics $g^{L}$ and $g^{N}$ on $L$ and $N$.

In view of the formal similarity of (8.1) and (8.2) with the construction by Ray-Singer [RS] of the analytic torsion, $\mathbb{B}\left(L, M, g^{M}\right)$ will be called a generalized analytic torsion form. 
If $E$ is a holomorphic vector bundle on $B$ equipped with a Hermitian metric $g^{E}$, if $\nabla^{E}$ is the corresponding holomorphic Hermitian connection, and if $R^{E}$ is the curvature of $\nabla^{E}$, we use the notation $\operatorname{Td}\left(g^{E}\right)$ instead of $\operatorname{Td}\left(-R^{E} / 2 i \pi\right)$.

Recall that the spaces of forms $P^{B}$ and $P^{B, 0}$ were already defined in Definition 4.5.

Theorem 8.3. The form $\mathbb{B}\left(L, M, g^{M}\right)$ lies in $P^{B}$. Moreover,

$$
\frac{\bar{\partial} \partial}{2 i \pi} \mathbb{B}\left(L, M, g^{M}\right)=\operatorname{Td}\left(g^{L}\right)-\frac{\operatorname{Td}\left(g^{M}\right)}{\operatorname{Td}\left(g^{N}\right)} .
$$

Proof. By Theorem 4.6, it is clear that the form $\mathbb{B}\left(L, M, g^{M}\right)$ lies in $P^{B}$. Since the form (8.3) is closed, using the double trangression formulas of Theorem 4.6 together with Theorems 4.8 and 7.7 , we get (8.5).

(b) An evaluation of the analytic torsion forms. From (6.2), it is clear that as $u \rightarrow+\infty$

$$
\varphi(u, x)=\frac{e^{\sqrt{u}}}{u}\left(1+O\left(\frac{1}{\sqrt{u}}\right)\right) .
$$

By using the analyticity of $\varphi(u, x)$ in the variable $x \in \mathbb{C}$, we deduce from (8.6) that as $u \rightarrow+\infty$

$$
\frac{\partial \varphi}{\partial x}(u, x)=\frac{e^{\sqrt{u}}}{u} O\left(\frac{1}{\sqrt{u}}\right) .
$$

From (8.6) and (8.7), we find that as $u \rightarrow+\infty$, for $|x|<2 \pi$

$$
\frac{\partial \varphi / \partial x}{\varphi}(u, x)=O\left(\frac{1}{\sqrt{u}}\right) \text {. }
$$

Of course, by (6.2), we know that

$$
\begin{aligned}
& \frac{\partial \varphi / \partial x}{\varphi}(u, x) \\
& \quad=\frac{1}{2}\left\{\frac{\left(\sinh \left(\sqrt{x^{2}+4 u} / 2\right)\right) x / \sqrt{x^{2}+4 u}-\sinh (x / 2)}{\cosh \left(\sqrt{x^{2}+4 u} / 2\right)-\cosh (x / 2)}\right\}
\end{aligned}
$$

from which (8.8) immediately follows.

Definition 8.4. For $s \in \mathbb{C}, 0<\operatorname{Re}(s)<\frac{1}{2}$, and $x \in \mathbb{C},|x|<2 \pi$, set

$$
C(s, x)=-\frac{1}{\Gamma(s)} \int_{0}^{+\infty} u^{s-1} \frac{\partial \varphi / \partial x}{\varphi}(u, x) d u \text {. }
$$

From (8.8), one immediately verifies that $s \rightarrow C(s, x)$ extends into a function which is holomorphic near $s=0$. Of course $C(s, x)$ is also holomorphic in the variable $x \in \mathbb{C},|x|<2 \pi$. Set

$$
D(x)=\frac{\partial C}{\partial s}(0, x) \text {. }
$$


Since $\varphi(u, x)$ is an even function of $x, C(s, x)$ and $D(x)$ are odd functions of $x$.

It is clear that $D(x)$ is a holomorphic function of $x \in \mathbb{C},|x|<2 \pi$. Then $\operatorname{Tr}\left[D\left(-R^{N} / 2 i \pi\right)\right]$ is a form in $P^{B}$. Moreover, by Bott-Chern [BoC, 3.28], the class of the form $\operatorname{Tr}\left[D\left(-R^{N} / 2 i \pi\right)\right]$ in $P^{B} / P^{B, 0}$ does not depend on the metric $g^{N}$. We will then denote by $D(N)$ the class of $\operatorname{Tr}\left[D\left(-R^{N} / 2 i \pi\right)\right]$ in $P^{B} / P^{B, 0}$. Similarly, we will denote by $\operatorname{Td}(L)$ the class of the form $\operatorname{Td}\left(g^{L}\right)$ in $P^{B} / P^{B, 0}$. This class does not depend either on the metric $g^{L}$.

Therefore, the class $\operatorname{Td}(L) D(N)$ is a well-defined element of $P^{B} / P^{B, 0}$.

Also by [BGS1, Theorem 1.29], to the holomorphic acyclic complex of Hermitian vector bundles

$$
0 \rightarrow L \underset{i}{\rightarrow} M \underset{j}{\rightarrow} N \rightarrow 0,
$$

we can associate a class of forms $\widetilde{\mathrm{Td}}\left(L, M, g^{M}\right)$ in $P^{B} / P^{B, 0}$ which verifies natural functorial properties, and which is such that

$$
\frac{\bar{\partial} \partial}{2 i \pi} \widetilde{\mathrm{Td}}\left(L, M, g^{M}\right)=\operatorname{Td}\left(g^{M}\right)-\operatorname{Td}\left(g^{L}\right) \operatorname{Td}\left(g^{N}\right) \text {. }
$$

The construction of [BGS1] generalizes a construction of Bott-Chern [BoC] and Donaldson [D].

Note that if the sequence (8.11) splits, i.e., if we have an identification of holomorphic Hermitian vector bundles $M=L \oplus N$ (where the metric on $L \oplus N$ is the orthogonal sum of the metrics $g^{L}$ and $g^{N}$ ) and the maps $L \rightarrow L \oplus$ $N$ and $L \oplus N \rightarrow N$ are the canonical injection and projection maps, then $\widetilde{\operatorname{Td}}\left(L, M, g^{M}\right)=0$.

We now prove the main result of this paper.

Theorem 8.5. The following identity holds:

$$
\mathbb{B}\left(L, M, g^{M}\right)=-\operatorname{Td}^{-1}\left(g^{N}\right) \widetilde{\mathrm{Td}}\left(L, M, g^{M}\right)+\operatorname{Td}(L) D(N) \text { in } P^{B} / P^{B, 0} .
$$

Proof. Let $\mathbb{P}^{1}$ be the one-dimensional complex projective plane equipped with two distinguished points $\{0\}$ and $\{\infty\}$, and with the meromorphic coordinate $z$.

By [BGS1, Theorem 1.29], or by using the Grassmann graph construction of Baum-Fulton-MacPherson [BaFM] of which a detailed exposition is given in [BGS5, §4], we can define a holomorphic acyclic chain complex on $B \times \mathbb{P}^{1}$,

$$
0 \rightarrow L^{\prime} \underset{i^{\prime}}{\rightarrow} M^{\prime} \underset{j^{\prime}}{\rightarrow} N^{\prime} \rightarrow 0
$$

which has the following two properties:

- On $B \times\{0\}=B$, the complex (8.14) coincides with the given complex (8.11).

- On $B \times\{\infty\}$, the complex (8.14) splits, i.e., $M^{\prime}=L^{\prime} \oplus N^{\prime}$, and the maps $L_{\mid B \times\{\infty\}}^{\prime} \rightarrow\left(L^{\prime} \oplus N^{\prime}\right)_{\mid B \times \infty}$ and $\left(L^{\prime} \oplus N^{\prime}\right)_{\mid B \times\{\infty\}} \rightarrow N_{\mid B \times\{\infty\}}^{\prime}$ are the canonical injection and projection maps. 
Let $g^{M^{\prime}}$ be a smooth Hermitian metric on $M^{\prime}$ which has the following two properties:

- On $B \times\{0\}, g^{M^{\prime}}$ coincides with the given metric $g^{M}$ on $M$.

- On $B \times\{\infty\}$, the splitting $M^{\prime}=L^{\prime} \oplus N^{\prime}$ is orthogonal, i.e., $L^{\prime}$ and $N^{\prime}$ are orthogonal in $M^{\prime}$.

Let $g^{L^{\prime}}$ and $g^{N^{\prime}}$ be the metrics on $L^{\prime}$ and $N^{\prime}$ induced by $g^{M^{\prime}}$ by the construction of $\S 2(\mathrm{~b})$. Of course, on $B \times\{0\}, g^{L^{\prime}}$ and $g^{N^{\prime}}$ coincide with $g^{L}$ and $g^{N}$.

On $\mathbb{P}^{1}$, we have the following classical equation of currents:

$$
\frac{\bar{\partial} \partial}{2 i \pi} \log |z|^{2}=\delta_{\{0\}}-\delta_{\{\infty\}} .
$$

By equations (8.5) and (8.12), we know that

$$
\begin{gathered}
\frac{\bar{\partial} \partial}{2 i \pi} \mathbb{B}\left(L^{\prime}, M^{\prime}, g^{M^{\prime}}\right)=\operatorname{Td}\left(g^{L^{\prime}}\right)-\frac{\operatorname{Td}\left(g^{M^{\prime}}\right)}{\operatorname{Td}\left(g^{N^{\prime}}\right)}, \\
\frac{\bar{\partial} \partial}{2 i \pi} \widetilde{\operatorname{Td}}\left(L^{\prime}, M^{\prime}, g^{M^{\prime}}\right)=\operatorname{Td}\left(g^{M^{\prime}}\right)-\operatorname{Td}\left(g^{L^{\prime}}\right) \operatorname{Td}\left(g^{N^{\prime}}\right) .
\end{gathered}
$$

Set

$$
\beta\left(L^{\prime}, M^{\prime}, g^{M^{\prime}}\right)=\mathbb{B}\left(L^{\prime}, M^{\prime}, g^{M^{\prime}}\right)+\operatorname{Td}^{-1}\left(g^{N^{\prime}}\right) \widetilde{\mathrm{Td}}\left(L^{\prime}, M^{\prime}, g^{M^{\prime}}\right) .
$$

By (8.16), we find that

$$
\frac{\bar{\partial} \partial}{2 i \pi} \beta\left(L^{\prime}, M^{\prime}, g^{M^{\prime}}\right)=0
$$

Also

$$
\begin{aligned}
\frac{\bar{\partial} \partial}{2 i \pi}\left(\log |z|^{2}\right) \beta\left(L^{\prime}, M^{\prime}, g^{M^{\prime}}\right)-\log |z|^{2} \frac{\bar{\partial} \partial}{2 i \pi} \beta\left(L^{\prime}, M^{\prime}, g^{M^{\prime}}\right) \\
=\frac{\bar{\partial}}{2 i \pi}\left(\partial\left(\log |z|^{2}\right) \beta\left(L^{\prime}, M^{\prime}, g^{M^{\prime}}\right)\right) \\
\quad+\frac{\partial}{2 i \pi}\left(\log |z|^{2} \bar{\partial} \beta\left(L^{\prime}, M^{\prime}, g^{M^{\prime}}\right)\right) .
\end{aligned}
$$

If $\alpha$ is a smooth form on $B \times \mathbb{P}^{1}$, let $\alpha_{0}$ and $\alpha_{\infty}$ denote the restriction of $\alpha$ to $B \times\{0\}$ and $B \times\{\infty\}$ respectively. Indentifying $B \times\{0\}$ and $B \times\{\infty\}$ with $B$, we consider $\alpha_{0}$ and $\alpha_{\infty}$ as smooth forms on $B$.

Using (8.15) and (8.18), and integrating (8.19) along the fiber of the map $B \times \mathbb{P}^{1} \rightarrow B$, we get

$$
\beta\left(L^{\prime}, M^{\prime}, g^{M^{\prime}}\right)_{0}-\beta\left(L^{\prime}, M^{\prime}, g^{M^{\prime}}\right)_{\infty} \in P^{B, 0} .
$$

Set

$$
\beta\left(L, M, g^{M}\right)=\mathbb{B}\left(L, M, g^{M}\right)+\operatorname{Td}^{-1}\left(g^{N}\right) \widetilde{\mathrm{Td}}\left(L, M, g^{M}\right) .
$$

Clearly,

$$
\beta\left(L^{\prime}, M^{\prime}, g^{M^{\prime}}\right)_{0}=\beta\left(L, M, g^{M}\right) .
$$


On the other hand, since the holomorphic Hermitian complex (8.14) splits on $B \times\{\infty\}$, by the considerations which follow equation (8.12), we know that

$$
\widetilde{\operatorname{Td}}\left(L^{\prime}, M^{\prime}, g^{M^{\prime}}\right)_{\infty}=0 \text { in } P^{B} / P^{B, 0} .
$$

We now calculate $\mathbb{B}\left(L^{\prime}, M^{\prime}, g^{M^{\prime}}\right)_{\infty}$. We use the formulas of Theorem 7.3 on $B \times\{\infty\}$, with the notation ' indicating that we are considering the complex (8.14). Since the complex (8.14) splits on $B \times\{\infty\}, R^{M^{\prime}}$ can be written in the form

$$
R^{M^{\prime}}=\left[\begin{array}{cc}
R^{L^{\prime}} & 0 \\
0 & R^{N^{\prime}}
\end{array}\right]
$$

Therefore by Theorem 7.3, we have the formula

$$
\begin{aligned}
\operatorname{Tr}_{s}[\exp ( & \left.\left.-\mathscr{B}_{u}^{\prime 2}+b N_{H}\right)\right]=\exp \left\{\frac{b \operatorname{dim} N}{2}-\frac{1}{2} \operatorname{Tr}\left[R^{L^{\prime}}\right]\right\} \\
& \times \frac{\operatorname{det}^{1 / 2}\left(I_{H_{N^{\prime}}}-\left(R^{N^{\prime}}+b I_{N^{\prime}}^{\prime}\right) J^{-1}-u J^{-2}\right)}{\operatorname{det}^{1 / 2}\left(I_{H_{N^{\prime}}}-R^{N^{\prime}} J^{-1}-u J^{-2}\right) \operatorname{det}^{1 / 2}\left(I_{H_{L^{\prime}}}-R^{L^{\prime} J^{-1}}\right)} \text { on } B \times\{\infty\} .
\end{aligned}
$$

By using (7.23) and (7.47), we find that

$$
\begin{aligned}
& \operatorname{Tr}_{s} {\left[\exp \left(-\mathscr{B}_{u}^{2}+b N_{H}\right)\right] } \\
&=\exp \left\{\frac{b \operatorname{dim} N}{2}\right\} \operatorname{Td}\left(-R^{L^{\prime}}\right) \frac{\Psi\left(u, R^{N^{\prime}}+b I_{N^{\prime}}^{\prime}\right)}{\Psi\left(u, R^{N^{\prime}}\right)} \text { on } B \times\{\infty\} .
\end{aligned}
$$

Note that in this split case, formula (8.25) could also have been derived by using Theorems 6.4 and 6.6 .

From (8.25), we deduce that

$$
\begin{aligned}
& \frac{\partial}{\partial b} \operatorname{Tr}_{s}\left[\exp \left(-\mathscr{B}_{u}^{\prime 2}+b N_{H}\right)\right]_{b=0} \\
& \quad=\frac{\operatorname{dim} N}{2} \operatorname{Td}\left(-R^{L^{\prime}}\right)+\operatorname{Td}\left(-R^{L^{\prime}}\right) \frac{\partial \Psi /\left.\partial b\left(u, R^{N^{\prime}}+b I_{N^{\prime}}^{\prime}\right)\right|_{b=0}}{\Psi\left(u, R^{N^{\prime}}\right)} \text { on } B \times\{\infty\} .
\end{aligned}
$$

By (6.15), the class $\Psi(u, \cdot)$ is multiplicative. The class

$$
\frac{\left[(\partial / \partial b) \Psi\left(u, R^{N^{\prime}}+b I_{N^{\prime}}^{\prime}\right)\right]_{b=0}}{\Psi\left(u, R^{N^{\prime}}\right)}
$$

is then additive. More precisely,

$$
\frac{\left[(\partial / \partial b) \Psi\left(u, R^{N^{\prime}}+b I_{N^{\prime}}^{\prime}\right)\right]_{b=0}}{\Psi\left(u, R^{N^{\prime}}\right)}=\operatorname{Tr}\left[\frac{(\partial \varphi / \partial x)}{\varphi}\left(u, R^{N^{\prime}}\right)\right] .
$$

If $[A(s)]^{\prime}$ denotes the form constructed in (8.1) associated with the Hermitian complex (8.14), we deduce from (8.1), (8.10), (8.26), and (8.27), that for $0<$ $\operatorname{Re}(s)<\frac{1}{2}$

$$
[A(s)]^{\prime}=-\operatorname{Td}\left(-R^{L^{\prime}}\right) \operatorname{Tr}\left[C\left(s, R^{N^{\prime}}\right)\right] \text { on } B \times\{\infty\} .
$$


From (8.28), we deduce that

$$
\mathbb{B}\left(L^{\prime}, M^{\prime}, g^{M^{\prime}}\right)_{\infty}=\left\{-\operatorname{Td}\left(\frac{-R^{L^{\prime}}}{2 i \pi}\right) \operatorname{Tr}\left[D\left(\frac{R^{N^{\prime}}}{2 i \pi}\right)\right]\right\}_{\infty} .
$$

Equivalently, since $D(x)$ is an odd function of $x$, we get

$$
\mathbb{B}\left(L^{\prime}, M^{\prime}, g^{M^{\prime}}\right)_{\infty}=\left\{\operatorname{Td}\left(\frac{-R^{L^{\prime}}}{2 i \pi}\right) \operatorname{Tr}\left[D\left(\frac{-R^{N^{\prime}}}{2 i \pi}\right)\right]\right\}_{\infty} .
$$

From (8.20), (8.22), (8.23), and (8.30), we get

$$
\begin{aligned}
\mathbb{B}\left(L, M, g^{M}\right)= & -\operatorname{Td}^{-1}\left(g^{N}\right) \widetilde{\operatorname{Td}}\left(L, M, g^{M}\right) \\
& +\left\{\operatorname{Td}\left(\frac{-R^{L^{\prime}}}{2 i \pi}\right) \operatorname{Tr}\left[D\left(\frac{-R^{N^{\prime}}}{2 i \pi}\right)\right]\right\}_{\infty} \text { in } P^{B} / P^{B, 0} .
\end{aligned}
$$

By the obvious analogue of (8.19), we know that

$$
\begin{aligned}
& \frac{\bar{\partial} \partial}{2 i \pi}\left(\log |z|^{2}\right) \operatorname{Td}\left(\frac{-R^{L^{\prime}}}{2 i \pi}\right) \operatorname{Tr}\left[D\left(\frac{-R^{N^{\prime}}}{2 i \pi}\right)\right] \\
& \quad=\frac{\bar{\partial}}{2 i \pi}\left(\partial\left(\log |z|^{2}\right) \operatorname{Td}\left(\frac{-R^{L^{\prime}}}{2 i \pi}\right) \operatorname{Tr}\left[D\left(\frac{-R^{N^{\prime}}}{2 i \pi}\right)\right]\right) .
\end{aligned}
$$

Using (8.15) and integrating (8.32) along the fiber of $B \times \mathbb{P}^{1} \rightarrow B$, we find that (8.33)

$$
\operatorname{Td}\left(\frac{-R^{L}}{2 i \pi}\right) \operatorname{Tr}\left[D\left(\frac{-R^{N}}{2 i \pi}\right)\right]-\left\{\operatorname{Td}\left(\frac{-R^{L^{\prime}}}{2 i \pi}\right) \operatorname{Tr}\left[D\left(\frac{-R^{N^{\prime}}}{2 i \pi}\right)\right]\right\}_{\infty} \in P^{B, 0}
$$

From (8.31) and (8.33), we obtain (8.13).

We now state the result proved in a joint Appendix with C. Soulé, which evaluates the function $D(x)$ explicitly.

Let $\zeta(s)=\sum_{1}^{+\infty} 1 / n^{s}$ be the Riemann zeta function.

Theorem 8.6. For $x \in \mathbb{C},|x|<2 \pi, D(x)$ is given by

$$
D(x)=\sum_{\substack{n \geq 1 \\ n \text { odd }}}\left(\Gamma^{\prime}(1)+\sum_{j=1}^{n} \frac{1}{j}+\frac{2 \zeta^{\prime}(-n)}{\zeta(-n)}\right) \zeta(-n) \frac{x^{n}}{n !} .
$$

Proof. This result is proved in the Appendix.

Remark 8.7. If $\widehat{A}(x)=(x / 2) / \sinh (x / 2)$, observe that for $|x|<2 \pi$

$$
\frac{\widehat{A}^{\prime}(x)}{\widehat{A}(x)}=\sum_{\substack{n \geq 1 \\ n \text { odd }}} \zeta(-n) \frac{x^{n}}{n !} .
$$

In fact, note that $C(0, x)=(-(\partial \varphi / \partial x) / \varphi)(0, x)$. Using (6.3), we find that

$$
C(0, x)=\frac{\widehat{A}^{\prime}(x)}{\widehat{A}(x)} \text {. }
$$


From equation (8) in the Appendix, we find that

$$
\frac{\widehat{A}^{\prime}(x)}{\widehat{A}(x)}=\sum_{k=1}^{+\infty} i\left(\left(2 k \pi\left(1-\frac{i x}{2 k \pi}\right)\right)^{-1}-\left(2 k \pi\left(1+\frac{i x}{2 k \pi}\right)\right)^{-1}\right) .
$$

Using equations (16) and (26) in the Appendix, we get (8.35).

Remark 8.8. Let $R(x)$ be the formal power series

$$
R(x)=\sum_{\substack{n \geq 1 \\ n \text { odd }}}\left(\sum_{j=1}^{n} \frac{1}{j}+\frac{2 \zeta^{\prime}(-n)}{\zeta(-n)}\right) \zeta(-n) \frac{x^{n}}{n !} .
$$

The formal power series $R(x)$ was introduced in Gillet-Soule [GS]. Gillet and Soulé conjectured in [GS] a Riemann-Roch-Grothendieck formula for arithmetic varieties. In this Riemann-Roch formula, the characteristic class $\sum_{1}^{n} R\left(x_{i}\right)$ should play a prominent role. It was obtained in [GS] by an explicit computation of the analytic torsion of the trivial line on $\mathbb{P}^{n}(\mathbb{C})$ equipped with the Fubini-Study metric.

By (8.34), (8.35), and (8.38), we find that

$$
D(x)=\Gamma^{\prime}(1) \frac{\widehat{A}^{\prime}(x)}{\widehat{A}(x)}+R(x) \text {. }
$$

\section{EQUIVARIANT INTERSECTION THEORY AND THE HARMONIC OSCILLATOR}

The purpose of this section is to perform some explicit nontrivial computations of differential forms on an acyclic complex

$$
0 \rightarrow E \underset{i}{\rightarrow} F \underset{j}{\rightarrow} G \rightarrow 0
$$

of holomorphic Hermitian equivariant vector bundles on a manifold $B$. The main idea is that our results of $\S \S 3-8$ can be partly formulated as an infinitedimensional analogue of these computations, where $E, F$, and $G$ are now certain Hilbert spaces of Fourier series in $L, M$, and $N$ respectively, equipped with the natural action of $S_{1}$ by translation in the time variable.

This fits with ideas outlined by Atiyah and Witten [A] and by the present author [B2] according to which there is an intimate relationship of the heat equation approach to index theory with equivariant cohomology on loop spaces. The point of view was also expanded by us in [B3] to include the Ray-Singer analytic torsion on complex manirolds in this framework.

So in some sense, this section gives a geometric counterpart to the superconnection operator theoretic analysis of $\S \S 3$ and 4 , and it puts the complicated calculations of $\S \S 7$ and 8 in the right geometric perspective. As pointed out in the Introduction, writing this section was essential in the writing of the entire paper.

This section is organized as follows. In (a), we construct certain equivariant differential forms on the total space of the exact sequence (9.1). By integration along the fiber, we obtain differential forms $\eta(u, b)$ which are the analogues of the forms $\operatorname{Tr}_{s}\left[\exp \left(-\mathscr{B}_{u}{ }^{2}+b N_{H}\right)\right]$. In (b), we calculate the asymptotics as $u \rightarrow 0$ 
and $u \rightarrow+\infty$ of $\eta(u, b)$. We thus recover formally Theorems 4.8 and 7.7. In (c), we explicitly evaluate the forms $\eta(u, b)$ and we recover formally the complicated formulas of Theorem 7.3. Finally in (d), we construct the infinitedimensional model which fits with the results of $\S \S 3-8$. In particular, we show that the form $\mathbb{B}\left(L, M, g^{M}\right)$ can be obtained by integration along the fiber of an infinite-dimensional Euler-Green current in the sense of our previous work with Gillet and Soule [BGS5] on geometric aspects of Arakelov theory. Also we obtain formula $(0.14)$ for $D(x)$.

A minor difference in the analogy with $\S \S 4-8$ is that our formal infinitedimensional results involve the $\hat{A}$ genus instead of the Todd genus. The reason for this is rather clear in view of $[\mathrm{A}]$. With the notation of $\S 3$, this means that $\Lambda\left(\bar{M}^{*}\right)$ should be replaced by $\Lambda\left(\bar{M}^{*}\right) \otimes \operatorname{det}(M)^{-1 / 2}$. Also we have refrained from explaining in depth the analogies between the finite- and infinitedimensional models, by simply concentrating on the extraordinary similarities of the formulas and of the results.

Formulas of Mathai-Quillen [MQ], Bismut [B5], and Bismut-Gillet-Soulé [BGS5] play an essential role in this section.

(a) Equivariant exact sequences and differential forms. Let $B$ be a compact connected complex manifold. Let

$$
0 \rightarrow E \underset{i}{\rightarrow} F \underset{j}{\rightarrow} G \rightarrow 0
$$

be a holomorphic acyclic chain complex of vector bundles. $E$ will be considered as a holomorphic subbundle of $F$, and $G$ is identified with $F / E$.

Let $g^{F}$ be a Hermitian metric on $F$. As in $\S 2(b), g^{F}$ induces smooth Hermitian metrics $g^{E}$ and $g^{G}$ on $F$ and $G$. Let $\nabla^{E}, \nabla^{F}$, and $\nabla^{G}$ be the corresponding holomorphic Hermitian connections on $E, F$, and $G$, and let $R^{E}, R^{F}$, and $R^{G}$ be their curvatures.

Let $J^{F}$ be a holomorphic skew-adjoint section of End $F$, which preserves $E$. Let $J^{E}$ be the restriction of $J^{F}$ to $E$ and let $J^{G}$ be the natural action of $J^{F}$ on $G$. Then $J^{E}$ and $J^{G}$ are also holomorphic skew-adjoint sections of End $E$ and End $G$. Moreover $J^{E}, J^{F}$, and $J^{G}$ are parallel with respect to the connections $\nabla^{E}, \nabla^{F}$, and $\nabla^{G}$.

The connection $\nabla^{F}$ defines a natural splitting of $T F$ into

$$
T F=F \oplus T^{H} F,
$$

where $T^{H} F$ is the horizontal subbundle of $T F$. We define $T^{H} E$ and $T^{H} G$ in a similar way.

If $y \in F$, we identify $y$ with $Y=y+\bar{y} \in F_{R}$. Then $J^{F} Y \in F_{R}$. Using (9.2), we consider $J^{F} Y=J^{F} y+J^{F} \bar{y}$ as a vertical holomorphic vector field on $F$. If $T B$ is equipped with a Hermitian metric, we can lift the metric of $T B$ to $T^{H} F$. We then equip $T F=F \oplus T^{H} F$ with the orthogonal sum of the metrics on $F$ and $T^{H} F$. Then $J^{F} Y$ is also a Killing vector field on $F$. Similarly, $J^{E} Y$ and $J^{G} Y$ will be considered as holomorphic Killing vector fields on $E$ and $G$. 
$E$ is a complex submanifold of $F$. The vector field $J^{F} Y$ restricts to the vector field $J^{E} Y$ on $E$. Similarly, the projection map $j: F \rightarrow G$ maps the vector field $J^{F} Y$ into $J^{G} j(Y)$.

If $Z \in T_{R} F$, let $Z^{V} \in F_{R}$ be the projection of $Z$ on $F_{R}$ with respect to the splitting (9.3) of $T_{R} F$. Let $\left(J^{F} Y\right)^{\prime}$ be the one-form on $F, Z \in T_{R} F \rightarrow$ $\left\langle J^{F} Y, Z^{V}\right\rangle$.

Similarly, we identify $J^{F}$ with the two-form

$$
Z, Z^{\prime} \in T_{R} F \rightarrow\left\langle Y^{V}, J^{F} Z^{V}\right\rangle \text {. }
$$

Let $i_{J^{F}}$ denote the interior multiplication by $J^{F} Y$ acting on $\Lambda\left(T_{R}^{*} F\right)$ and let $L_{J^{F} Y}$ be the Lie derivative operator associated with the vector field $J^{F} Y$. Then the operators $d, i_{J^{F} Y}$, and $L_{J^{F} Y}$ act on the set of smooth sections of $\Lambda\left(T_{R}^{*} F\right)$. Moreover,

$$
L_{J^{F} Y}=\left(d+i_{J^{F} Y}\right)^{2}
$$

Let $\pi$ be any of the projections $E, F, G \rightarrow B$. If $\omega$ is a form on $B$, we identify $\omega$ with the form $\pi^{*} \omega$ on $E, F$, or $G$.

Proposition 9.1. The following identities hold:

$$
\begin{gathered}
-\frac{1}{2}\left(d+i_{J^{F} Y}\right)\left(J^{F} Y\right)^{\prime}=-\frac{\left|J^{F} Y\right|^{2}}{2}+\frac{1}{2}\left\langle R^{F} J^{F} Y, Y\right\rangle+J^{F}, \\
L_{J^{F} Y}\left(J^{F} Y\right)^{\prime}=0 .
\end{gathered}
$$

Proof. Let $\nabla$ be an arbitrary torsion free connection on $T_{R} B$. We identify $T^{H} F$ with $\pi^{*} T B$. Then the connection $\nabla$ lifts to $T_{R}^{H} F$. Let $\nabla^{\prime}=\nabla^{F} \oplus \nabla$ be the sum of the connections $\nabla^{F}$ and $\nabla$ on $T_{R} F=F_{R} \oplus T_{R}^{H} F$. Then $\nabla^{\prime}$ maps smooth sections of $\Lambda^{p}\left(T_{R}^{*} F\right)$ into smooth sections of $\Lambda^{1}\left(T_{R}^{*} F\right) \otimes \Lambda^{p}\left(T_{R}^{*} F\right)$. By antisymmetrization in all indices, we then get an operator ${ }^{a} \nabla^{\prime}$ which maps smooth section of $\Lambda^{p}\left(T_{R}^{*} F\right)$ into smooth sections of $\Lambda^{p+1}\left(T_{R}^{*} F\right)$.

By [BGS5, Proposition 3.6], we know that

$$
d={ }^{a} \nabla^{\prime}+i_{R^{F} Y} .
$$

Since the form $\left(J^{F} Y\right)^{\prime}$ is vertical, and since $\nabla^{F} J^{F}=0$, we deduce from (9.7) that

$$
d\left(J^{F} Y\right)^{\prime}=-2 J^{F}+\left\langle R^{F} Y, J^{F} Y\right\rangle .
$$

Since $\nabla^{F} J^{F}=0$, we know that $\left[R^{F}, J^{F}\right]=0$ and so

$$
\left\langle R^{F} Y, J^{F} Y\right\rangle=-\left\langle R^{F} J^{F} Y, Y\right\rangle \text {. }
$$

Using (9.8) and (9.9), we get the first line of (9.6). Since $J^{F} Y$ is a Killing vector field, we find that $L_{J^{F} Y}\left(J^{F} Y\right)^{\prime}=0$. Our proposition is proved.

Definition 9.2. Let $\alpha$ be the smooth even form on $F$,

$$
\alpha=(2 \pi)^{-\operatorname{dim} F} \exp \left\{-\frac{\left|J^{F} Y\right|^{2}}{2}+\frac{1}{2}\left\langle R^{F} J^{F} Y, Y\right\rangle+J^{F}\right\} .
$$


The form $\alpha$ was introduced in a different context in [B2, proof of Theorem 1.3].

We define the space of differential forms $P^{F}$ as in Definition 4.5.

Theorem 9.3. The form $\alpha$ lies in $P^{F}$. Also

$$
\left(d+i_{J^{F} Y}\right) \alpha=0 .
$$

Proof. Since $R^{F}$ and $J^{F}$ are of type $(1,1)$, it is clear that $\alpha \in P^{F}$. By Proposition 9.1, we know that

$$
\alpha=(2 \pi)^{-\operatorname{dim} F} \exp \left\{-\frac{1}{2}\left(d+i_{J^{F}}\right)\left(J^{F} Y\right)^{\prime}\right\} .
$$

Then

$$
\left(d+i_{J^{F} Y}\right) \alpha=-\frac{1}{2}\left(\left(d+i_{J^{F} Y}\right)^{2}\left(J^{F} Y\right)^{\prime}\right) \alpha .
$$

Since $\left(d+i_{J^{F} Y}\right)^{2}=L_{J^{F} Y}$, and since $L_{J^{F} Y}\left(J^{F} Y\right)^{\prime}=0$, by Proposition 9.1, we get $(9.11)$.

On the total space of $G$, we can reproduce what has been done in (9.3). The splitting (9.3) is now replaced by

$$
T G=G \oplus T^{H} G .
$$

Observe that since $J^{G}$ is a holomorphic skew-adjoint section of $\operatorname{End}(G)$, then

$$
\left(\bar{\partial}+i_{J^{G}}\right)^{2}=0, \quad\left(\partial+i_{J^{G} \bar{y}}\right)^{2}=0 .
$$

From (9.14), and from the analogue of (9.5), we find that

$$
L_{J^{G} Y}=\left[\bar{\partial}+i_{J^{G} y}, \partial+i_{J^{G}}\right]
$$

(where [ , ] still denotes a supercommutator).

If $A \in \operatorname{End}\left(G_{R}\right) \otimes_{R} \mathbb{C}$ is antisymmetric, we identify $A$ with the two-form $U, V \in T_{R} G \rightarrow\langle U, A V\rangle$. Let $I_{G}^{\prime} \in \operatorname{End}\left(G_{R}\right) \otimes_{R} \mathbb{C}$ act like +1 on $G$, and -1 on $\bar{G}$. $I_{G}^{\prime}$ is clearly antisymmetric.

We now use the conventions of Mathai-Quillen [MQ] combined with the conventions of Bismut-Gillet-Soule [BGS5, §3]. The essential difference with [BGS5] is that we will work with equivariant characteristic classes or forms.

If $A \in \operatorname{End}\left(G_{R}\right) \otimes_{R} \mathbb{C}$ is antisymmetric, let $\operatorname{Pf}(A)$ denote the Pfaffian of $A$. If $A$ is invertible, then $A^{-1}$ is a two-form on the fibers of $G_{R}$, and $\operatorname{Pf}(A) \exp \left\{-|Y|^{2} / 2+A^{-1}\right\}$ is a series of forms on the fibers of $G_{R}$. By MathaiQuillen [MQ], this form can be extended by continuity to noninvertible antisymmetric $A$. As in usual Chern-Weil theory, we can replace $A$ by $J^{G}+R^{G}+b I_{G}^{\prime}$ and get (by antisymmetrization in all indices) a form on the total space of $G$.

Definition 9.4. For $u \geq 0$ and $b \in R$, let $\beta(u, b)$ be the following form on $G$ :

$$
\beta(u, b)=\operatorname{Pf}\left(J^{G}+R^{G}+b I_{G}^{\prime}\right) \exp \left\{-u\left(\frac{|Y|^{2}}{2}+\left(J^{G}+R^{G}+b I_{G}^{\prime}\right)^{-1}\right)\right\}
$$


Set

$$
\gamma(u)=\beta(u, 0), \quad \varepsilon(u)=\frac{\partial}{\partial b}[\beta(u, b)]_{b=0}
$$

We now recall a fundamental result of [BGS5].

Theorem 9.5. The forms $\beta(u, b), \gamma(u)$, and $\varepsilon(u)$ lie in $P^{G}$. For any $u \geq 0$,

$$
\begin{gathered}
\left(d+i_{J^{G} Y}\right) \gamma(u)=0, \\
\frac{\partial}{\partial u} \gamma(u)=\frac{1}{u}\left(\bar{\partial}+i_{J^{G} y}\right)\left(\partial+i_{J^{G}}\right) \varepsilon(u) .
\end{gathered}
$$

Proof. The fact that the forms $\beta(u, b), \gamma(u)$, and $\varepsilon(u)$ lie in $P^{G}$ is easy to prove. The identities (9.18) essentially follow from Bismut-Gillet-Soule [BGS5, Theorem 3.10], where the curvature $R^{G}$ has to be replaced by the equivariant curvature $J^{G}+R^{G}$.

Since $J^{F}$ is a holomorphic skew-adjoint parallel section of End $F$, one verifies easily that $F$ splits holomorphically and orthogonally into

$$
F=\bigoplus_{\lambda \in \Lambda} F_{\lambda}
$$

In (9.19), $\Lambda$ is a finite set of distinct purely imaginary complex numbers and the $F_{\lambda}$ are nonzero holomorphic vector bundles. Moreover for any $\lambda \in \Lambda, J^{F}$ acts on $F_{\lambda}$ by multiplication by $\lambda$.

The acyclic complex (9.1) then splits into a direct sum of acyclic complexes:

$$
0 \rightarrow E_{\lambda} \underset{i}{\rightarrow} F_{\lambda} \underset{j}{\rightarrow} G_{\lambda} \rightarrow 0 \text {. }
$$

Again $J^{E}$ and $J^{G}$ act on $E_{\lambda}$ and $G_{\lambda}$ by multiplication by $\lambda$.

We now make two basic assumptions:

(1) $\{0\} \in \Lambda$;

(2) $E_{\{0\}}=\{0\}$.

Observe that if $Y \in F, J^{F} Y=0$, and $j(Y)=0$, then $Y=0$.

Therefore, for any $u>0$ and $b \in R$, the forms $\alpha j^{*} \beta(u, b), \alpha j^{*} \gamma(u)$, and $\alpha j^{*} \varepsilon(u)$ on $F$ are Gaussian shaped, i.e., they exhibit a Gaussian like decay as $|Y| \rightarrow+\infty$.

In the sequel, $\int_{F}$ denotes integration along the fiber of $\pi: F \rightarrow B$.

Definition 9.6. For $u>0$ and $b \in R$, let $\theta(u), \eta(u, b)$, and $\chi(u)$ be the following forms on $B$ :

$$
\theta(u)=\int_{F} \alpha j^{*} \gamma(u), \quad \eta(u, b)=\int_{F} \alpha j^{*} \beta(u, b), \quad \chi(u)=\int_{F} \alpha j^{*} \varepsilon(u) .
$$

Theorem 9.7. The forms $\theta(u), \eta(u, b)$, and $\chi(u)$ lie in $P^{B}$. The forms $\theta(u)$ are closed and their cohomology class does not depend on $u$. More precisely,

$$
\frac{\partial}{\partial u} \theta(u)=\frac{1}{u} \bar{\partial} \partial \chi(u)
$$


Proof. By Theorems 9.3 and 9.5, it is clear that the considered forms lie in $P^{B}$. Also

$$
d \theta(u)=\int_{F} d\left(\alpha j^{*} \gamma(u)\right) .
$$

Since $J^{F} Y \in F_{R}$ for $Y \in F_{R}$, it is clear that $\int_{F} i_{J^{F} Y}\left(\alpha j^{*} \gamma(u)\right)=0$. Therefore we can rewrite (9.23) in the form

$$
d \theta(u)=\int_{F}\left(d+i_{J^{F} Y}\right)\left(\alpha j^{*} \gamma(u)\right) .
$$

Now by Theorems 9.3 and 9.5 , we get

$$
\left(d+i_{J^{F} Y}\right)\left(\alpha j^{*} \gamma(u)\right)=0 .
$$

From (9.24) and (9.25) we deduce that $d \theta(u)=0$.

Using Theorem 9.5, we know that

$$
\frac{\partial}{\partial u} \theta(u)=\frac{1}{u} \int_{F} \alpha\left(\bar{\partial}+i_{J^{F} y}\right)\left(\partial+i_{J^{F} \bar{y}}\right) j^{*} \varepsilon(u) .
$$

Since $\alpha \in P^{F}$, and since $\left(d+i_{J^{F} Y}\right) \alpha=0$ by Theorem 9.3 , we find that

$$
\left(\bar{\partial}+i_{J^{F} y}\right) \alpha=0, \quad\left(\partial+i_{J^{F} \bar{y}}\right) \alpha=0 .
$$

(9.22) now follows from (9.26) and (9.27).

Remark 9.8. Theorem 9.7 should be understood as a finite-dimensional analogue of Theorem 4.6.

(b) The asymptotics of the forms $\eta(u, b)$. Let $F^{\perp}$ and $G^{\perp}$ be the orthogonal bundles to $F_{\{0\}}$ and $G_{\{0\}}$ in $F$ and $G . F^{\perp}$ and $G^{\perp}$ are holomorphic Hermitian vector bundles.

In the sequel, we will often write $J$ instead of $J^{E}, J^{F}$ or $J^{G}$. Also $\operatorname{Pf}_{F_{R}^{\perp}}\left(J+R^{F}\right)$ and $\operatorname{Pf}_{G_{R}^{\perp}}\left(J+R^{G}+b I_{G}^{\prime}\right)$ denote the Pfaffians of the restrictions of $J+R^{F}$ and $J+R^{G}+b I_{G}^{\prime}$ to $F_{R}^{\perp}$ and $G_{R}^{\perp}$ respectively.

Theorem 9.9. As $u \rightarrow 0$

$$
\eta(u, b)=\frac{\operatorname{Pf}_{G_{R}^{\perp}}\left(J+R^{G}+b I_{G}^{\prime}\right)}{\operatorname{Pf}_{F_{R}^{\perp}}\left(J+R^{F}\right)}+O(u) .
$$

As $u \rightarrow+\infty$

$$
\eta(u, b)=\frac{1}{\operatorname{Pf}_{E_{R}}\left(J+R^{E}\right)}+O\left(\frac{1}{u}\right) .
$$

Proof. Using our fundamental assumptions, one verifies easily that if $k$ is the embedding $G_{\{0\}} \rightarrow G$

$$
\lim _{u \downarrow 0} \eta(u, b)=\int_{F^{\perp}} \alpha \int_{G_{\{0\}}} k^{*} \beta(0, b) .
$$


Now by proceeding as in [B2, Theorem 1.3], we find that

$$
\int_{F^{\perp}} \alpha=\frac{(2 \pi)^{-\operatorname{dim} F_{\{0\}}}}{\operatorname{Pf}_{F_{R}^{\perp}}\left(J+R^{F}\right)} .
$$

Similarly,

$$
\begin{aligned}
\int_{G_{\{0\}}} k^{*} \beta(0, b) & =\frac{(2 \pi)^{\operatorname{dim} G_{\{0\}} \operatorname{Pf}_{G_{R}}\left(J+R^{G}+b I_{G}^{\prime}\right)}}{\operatorname{Pf}_{G_{R^{\prime 0\}}}}\left(R^{G}+b I_{G}^{\prime}\right)} \\
& =(2 \pi)^{\operatorname{dim} G_{\{0\}} \operatorname{Pf}_{G_{R}^{\perp}}\left(J+R^{G}+b I_{G}^{\prime}\right) .}
\end{aligned}
$$

Since $\operatorname{dim} F_{\{0\}}=\operatorname{dim} G_{\{0\}}$, we deduce from (9.30)-(9.32) that

$$
\lim _{u \downarrow 0} \eta(u, b)=\frac{\operatorname{Pf}_{G_{R}^{\perp}}\left(J+R^{G}+b I_{G}^{\prime}\right)}{\operatorname{Pf}_{F_{R}^{\perp}}\left(J+R^{F}\right)}
$$

Similarly,

$$
\lim _{u \rightarrow+\infty} \eta(u, b)=\int_{E} i^{*} \alpha \int_{G} \beta(1, b) .
$$

Now

$$
\begin{aligned}
i^{*}\left(d+i_{J^{F} Y}\right)\left(J^{F} Y\right)^{\prime} & =d i^{*}\left(J^{F} Y\right)^{\prime}+\left|J^{E} Y\right|^{2} \\
& =d\left(J^{E} Y\right)^{\prime}+\left|J^{E} Y\right|^{2}=\left(d+i_{J^{E} Y}\right)\left(J^{E} Y\right)^{\prime} .
\end{aligned}
$$

Then, by proceeding as in [B2, Theorem 1.3], we get

$$
\begin{aligned}
& \int_{E} i^{*} \alpha= \frac{1}{(2 \pi)^{\operatorname{dim} F}} \int_{E} \exp \left\{-\frac{1}{2}\left(d+i_{J^{E} Y}\right)\left(J^{E} Y\right)^{\prime}\right\} \\
&= \frac{1}{(2 \pi)^{\operatorname{dim} F-\operatorname{dim} E} \mathrm{Pf}_{E_{R}}\left(J+R^{E}\right)}, \\
& \int_{G} \beta(1, b)=(2 \pi)^{\operatorname{dim} G} .
\end{aligned}
$$

Since $\operatorname{dim} E-\operatorname{dim} F+\operatorname{dim} G=0$, we deduce from (9.34) and (9.36) that

$$
\lim _{u \rightarrow+\infty} \eta(u, b)=\frac{1}{\operatorname{Pf}_{E_{R}}\left(J+R^{E}\right)} .
$$

The estimates of the remainders in (9.28) and (9.29) are easy and are left to the reader.

Remark 9.10. (9.28) is the analogue of (4.71) and (9.29) is the analogue of (7.73). 
(c) An evaluation of the forms $\eta(u, b)$. Recall that $G$ is identified with the orthogonal to $E$ in $F$. We then have the identification of smooth vector bundles $F=E \oplus G$. Let $P^{E}$ and $P^{G}$ denote the orthogonal projection operator from $F$ onto $E$ and $G$. Let $\nabla^{\prime}=\nabla^{E} \oplus \nabla^{G}$ be the sum of the connections $\nabla^{E}$ and $\nabla^{G}$ on $F=E \oplus G$. Set

$$
A=\nabla^{F}-\nabla^{\prime} \text {. }
$$

$A$ is a one-form on $B$ with values in skew-adjoint elements of End $F$, which exchange $E$ and $G$. On $F_{R}^{\perp}$, the operator $-J^{2}+u P^{G}$ is self-adjoint and positive definite. If $C \in \Lambda^{\text {even }}\left(T_{R}^{*} B\right) \otimes$ End $F_{R}^{\perp}$, we can then unambiguously define $\operatorname{det}_{F_{R}^{\perp}}^{1 / 2}\left(-J^{2}+u P^{G}+C\right)$. The same considerations can also be used on $G_{R}^{\perp}$.

Theorem 9.11. For any $u>0$ and $b \in R$, the following identity holds:

$$
\eta(u, b)=\frac{\operatorname{Pf}_{E_{R}}(J) \operatorname{det}_{G}^{1 / 2}\left(-J^{2}-\left(R^{G}+b I_{G}^{\prime}\right) J+u I_{G}\right)}{\operatorname{det}_{F_{R}^{\perp}}^{1 / 2}\left(-J^{2}-R^{F} J+u P^{G}-u P^{E} A\left(J^{2}+\left(R^{G}+b I_{G}^{\prime}\right) J-u I_{G}\right)^{-1} A P^{E} J\right)} .
$$

Proof. We first assume that $b=0$. Clearly,

$$
\begin{aligned}
(9.40) \eta(u, 0)= & (2 \pi)^{-\operatorname{dim} F} \int_{F} \exp \left\{-\frac{\left|J^{F} Y\right|^{2}}{2}+\frac{1}{2}\left\langle R^{F} J^{F} Y, Y\right\rangle+J^{F}\right\} \\
& \times \operatorname{Pf}\left(J^{G}+R^{G}\right) \exp \left\{-u\left(\frac{\left|P^{G} Y\right|^{2}}{2}+j^{*}\left(J^{G}+R^{G}\right)^{-1}\right)\right\} .
\end{aligned}
$$

We will often use the splitting $F=F_{\{0\}} \oplus F^{\perp}, G=G_{\{0\}} \oplus G^{\perp}$ to express the integral in (9.40) as a product of integrals. In particular,

$$
\begin{aligned}
\eta(u, 0)= & (2 \pi)^{-\operatorname{dim} G_{0}} \int_{G_{0}} \operatorname{Pf}_{G_{R\{0\}}}\left(R^{G}\right) \exp \left\{-u\left(\frac{|Y|^{2}}{2}+\left(R^{G}\right)^{-1}\right)\right\} \\
& \times(2 \pi)^{-\operatorname{dim} F^{\perp}} \int_{F^{\perp}} \exp \left\{-\frac{\left|J^{F} Y\right|^{2}}{2}+\frac{1}{2}\left\langle R^{F} J^{F} Y, Y\right\rangle+J^{F}\right\} \\
& \times \operatorname{Pf}_{G_{R}^{\perp}}\left(J^{G}+R^{G}\right) \exp \left\{-u\left(\frac{\left|P^{G} Y\right|^{2}}{2}+j^{*}\left(J^{G}+R^{G}\right)^{-1}\right)\right\} .
\end{aligned}
$$

By $[\mathrm{MQ}, \S 4]$

$$
(2 \pi)^{-\operatorname{dim} G_{0}} \int_{G_{0}} \operatorname{Pf}_{G_{R\{0\}}}\left(R^{G}\right) \exp \left(-u\left(\frac{|Y|^{2}}{2}+\left(R^{G}\right)^{-1}\right)\right)=1 .
$$

Now the key point is that the vertical two-form $J^{F}$ is calculated using the connection $\nabla^{F}$, while the vertical two-form $j^{*}\left(J^{G}+R^{G}\right)^{-1}$ is calculated with the connection $\nabla^{G}$. To eliminate this discrepancy, we will proceed as in [B5, $\S 5]$.

If $X \in T_{R} F$, let $D_{X} Y$ denote its projection on $F_{R}$ associated with the splitting of $T_{R} F=F_{R} \oplus T_{R}^{H} F$. Similarly, the connection $\nabla^{\prime}$ also defines a 
splitting of $T_{R} F$. If $X \in T_{R} F$, let $D_{X}^{\prime} Y$ denote the corresponding projection on $F_{R}$. Clearly,

$$
D_{X} Y=D_{X}^{\prime} Y+A\left(\pi_{*} X\right) Y \text {. }
$$

In the sequel, we consider $D Y, D^{\prime} Y$ as one-forms on $F$ taking values in $F_{R}$. Then

$$
\begin{aligned}
J_{F} & =\frac{1}{2}\left\langle D Y, J^{F} D Y\right\rangle \text { on } F^{\perp}, \\
j^{*}\left(J^{G}+R^{G}\right)^{-1} & =\frac{1}{2}\left\langle P^{G} D^{\prime} Y,\left(J^{G}+R^{G}\right)^{-1} P^{G} D^{\prime} Y\right\rangle \text { on } F^{\perp} .
\end{aligned}
$$

Observe that the operator $J^{F}-u j^{*}\left(J^{G}+R^{G}\right)^{-1}$ is formally invertible on $F^{\perp}$. From (9.43), we have the identities of forms on $F^{\perp}$ :

(9.44)

$$
\begin{aligned}
J^{F}- & u j^{*}\left(J^{G}+R^{G}\right)^{-1} \\
= & \frac{1}{2}\left\langle D^{\prime} Y+A Y, J^{F}\left(D^{\prime} Y+A Y\right)\right\rangle-\frac{u}{2}\left\langle P^{G} D^{\prime} Y,\left(J^{G}+R^{G}\right)^{-1} P^{G} D^{\prime} Y\right\rangle \\
= & -\frac{1}{2}\left\langle A^{2} J^{F} Y, Y\right\rangle+\frac{1}{2}\left\langle D^{\prime} Y+\left(J^{F}-u P^{G}\left(J^{G}+R^{G}\right)^{-1} P^{G}\right)^{-1} J^{F} A Y,\right. \\
& \left.\left(J^{F}-u P^{G}\left(J^{G}+R^{G}\right)^{-1} P^{G}\right)\left(D^{\prime} Y+\left(J^{F}-u P^{G}\left(J^{G}+R^{G}\right)^{-1}\right) P^{G}\right)^{-1} J^{F} A Y\right\rangle \\
& -\frac{1}{2}\left\langle\left(J^{F}-u P^{G}\left(J^{G}+R^{G}\right)^{-1} P^{G}\right)^{-1} J^{F} A Y, J^{F} A Y\right\rangle .
\end{aligned}
$$

Since $A$ maps $G_{R}$ into $E_{R}$, we find that

$$
\begin{aligned}
& -\frac{1}{2}\left\langle\left(J^{F}-u P^{G}\left(J^{G}+R^{G}\right) P^{G}\right)^{-1} J^{F} A P^{G} Y, J^{F} A P^{G} Y\right\rangle \\
& \quad=\frac{1}{2}\left\langle A^{2} J^{F} P^{G} Y, P^{G} Y\right\rangle .
\end{aligned}
$$

From (9.44) and (9.45), we thus get the identity of forms on $F^{\perp}$ :

$$
\begin{aligned}
J^{F}-u j^{*}\left(J^{G}+R^{G}\right)^{-1} & \\
= & \frac{1}{2} u\left\langle P^{E} A\left(\left(J^{G}\right)^{2}+R^{G} J^{G}-u I_{G}\right)^{-1} A P^{E} J^{F} Y, P^{E} Y\right\rangle \\
& +\frac{1}{2}\left\langle D^{\prime} Y+\left(J^{F}-u P^{G}\left(J^{G}+R^{G}\right)^{-1} P^{G}\right)^{-1} J^{F} A Y,\right. \\
& \left(J^{F}-u P^{G}\left(J^{G}+R^{G}\right)^{-1} P^{G}\right) \\
& \times\left(D^{\prime} Y+\left(J^{F}-u P^{G}\left(J^{G}+R^{G}\right)^{-1} P^{G}\right)^{-1} J^{F} A Y\right\rangle .
\end{aligned}
$$

When integrating along the fiber $F^{\perp}$, all the vertical Grassmann variables are saturated. We deduce from (9.41), (9.42), and (9.46) that

$$
\begin{aligned}
& \eta(u, 0)=\int_{F^{\perp}} \exp \left\{\frac { 1 } { 2 } \left\langle\left(\left(J^{F}\right)^{2}+\right.\right.\right. R^{F} J^{F} \\
&\left.\left.+u P^{E} A\left(\left(J^{G}\right)^{2}+R^{G} J^{G}-u I_{G}\right)^{-1} A P^{E} J^{F}-u P^{G}\right) Y, Y\right\rangle \\
&\left.\quad+J^{F}-u P^{G}\left(J^{G}+R^{G}\right)^{-1} P^{G}\right\} \operatorname{Pf}_{G_{R}^{\perp}}\left[J^{G}+R^{G}\right] .
\end{aligned}
$$

To calculate (9.47), we proceed as in [B2, proof of Theorem 1.3]. Since on $F_{R}^{\perp},-\left(J^{F}\right)^{2}$ is a positive operator, we find that

$$
\eta(u, 0)=\frac{\operatorname{Pf}_{F_{R}^{\perp}}\left(J^{F}-u P^{G}\left(J^{G}+R^{G}\right)^{-1} P^{G}\right) \mathbf{P f}_{G_{R}^{\frac{1}{R}}}\left[J^{G}+R^{G}\right]}{\operatorname{det}_{F_{R}^{1}}^{1 / 2}\left(-\left(J^{F}\right)^{2}-R^{F} J^{F}+u P^{G}-u P^{E} A\left(\left(J^{G}\right)^{2}+R^{G} J^{G}-u I_{G}\right)^{-1} A P^{E} J^{F}\right)} .
$$


Now one easily verifies that

$$
\begin{gathered}
\operatorname{Pf}_{F_{R}^{\perp}}\left(J^{F}-u P^{G}\left(J^{G}+R^{G}\right)^{-1} P^{G}\right) \operatorname{Pf}_{G_{R}^{\perp}}\left(J^{G}+R^{G}\right) \\
=\operatorname{Pf}\left(J^{E}\right) \operatorname{det}_{G_{R}^{\perp}}^{1 / 2}\left(-\left(J^{G}\right)^{2}-R^{G} J^{G}+u I_{G}\right) .
\end{gathered}
$$

(9.39) now follows from (9.48) and (9.49). Replacing $R^{G}$ by $R^{G}+b I_{G}^{\prime}$, we obtain (9.39) in full generality.

Remark 9.12. The double transgression formulas (9.22) are not easily derived from formula (9.39).

Also note that (9.39) can be rewritten in the form

$$
\eta(u, b)=\frac{\operatorname{sgn}\left(\operatorname{Pf}_{E_{R}}(J)\right) \operatorname{det}_{G}^{1 / 2}\left(I_{G}+\left(R^{G}+b I_{G}^{\prime}\right) J^{-1}-u J^{-2}\right)}{\operatorname{det}_{F_{R}^{1}}^{1 / 2}\left(I_{F}+R^{F} J^{-1}-u P^{G} J^{-2}+u P^{E} A\left(J^{2}+\left(R^{G}+b I_{G}^{\prime}\right) J-u I_{G}\right)^{-1} A P^{E} J^{-1}\right)} .
$$

Using the fact that the determinant of a matrix coincides with the determinant of its transpose, we get

$$
\eta(u, b)=\frac{\operatorname{sgn}\left(\mathrm{Pf}_{E_{R}}(J)\right) \operatorname{det}_{G}^{1 / 2}\left(I_{G}-\left(R^{G}+b I_{G}^{\prime}\right) J^{-1}-u J^{-2}\right)}{\operatorname{det}_{F_{R}^{\perp}}^{1 / 2}\left(I_{F}-R^{F} J^{-1}-u P^{G} J^{-2}-u P^{E} A\left(J^{2}-\left(R^{G}+b I_{G}^{\prime}\right) J-u I_{G}\right)^{-1} A P^{E} J^{-1}\right)} .
$$

At this stage, the similarity of formula (9.51) with formula (7.24) for $\operatorname{Tr}_{s}\left[\exp \left(-\mathscr{B}_{u}^{2}+b N_{H}\right)\right]$ should be obvious.

(d) Fourier series and generalized analytic torsion forms. We now use again the notation of $\S \S 3-8$. Set

$$
E=H_{L}, \quad F=H_{M} \oplus N, \quad G=L_{N}^{2} .
$$

We then have an obvious exact sequence of infinite-dimensional holomorphic Hermitian vector bundles on $B$ :

$$
0 \rightarrow E \underset{i}{\rightarrow} F \underset{j}{\rightarrow} G \rightarrow 0 .
$$

Also the operator $J=d / d t$ acts as an unbounded operator on $E, F$, and $G$.

One easily verifies that the assumptions of $\S 9(a)$ are verified in an infinitedimensional context.

The comparison of Theorems 4.6, 4.8, 7.3, and 7.7 with Theorems 9.7, 9.9, and 9.11 should make clear that the results of $\S \S 3-8$ are infinite-dimensional analogues of well-defined finite-dimensional results. The only unsubstantial difference is that in the infinite-dimensional analogue, spinors are considered instead of forms, which accounts for the fact that factors like $\exp \left\{-\frac{1}{2} \operatorname{Tr}\left[R^{L}\right]\right\}$ do not appear.

In the context of spinors, the form $\operatorname{Tr}_{s}\left[\exp \left(-\mathscr{B}_{u}^{2}+b\left(N_{H}-(\operatorname{dim} N) / 2\right)\right)\right]$ is the analogue of $\eta(u, b)$. Using (9.42), we find that the form $A^{\prime}(0)$ which was 
defined in (8.1) and (8.2) can then be written formally as

$$
\begin{aligned}
A^{\prime}(0)= & \frac{\operatorname{Pf}_{E_{R}}(J)}{(2 \pi)^{\operatorname{dim} F^{\perp}}} \int_{F^{\perp}} \exp \left\{-\frac{\left|J^{F} Y\right|^{2}}{2}+\frac{1}{2}\left\langle R^{F} J^{F} Y, Y\right\rangle+J^{F}\right\} \\
& \times\left(-\frac{\partial}{\partial b}\left\{\operatorname{Pf}\left[J^{G}+R^{G}+b I_{G}^{\prime}\right]\right.\right. \\
& \left.\left.\times \log \left(\frac{\left|P^{G} Y\right|^{2}}{2}+\left(J^{G}+R^{G}+b I_{G}^{\prime}\right)^{-1}\right)\right\}_{b=0}\right) .
\end{aligned}
$$

Of course, in the context of $\S 8, \operatorname{dim} F=+\infty$.

In the terminology of Bismut-Gillet-Soulé [BGS5, §3f)]

$$
\tilde{e}=-\frac{\partial}{\partial b}\left\{\operatorname{Pf}\left(J^{G}+R^{G}+b I_{G}^{\prime}\right) \log \left(\frac{|Y|^{2}}{2}+\left(J^{G}+R^{G}+b I_{G}^{\prime}\right)^{-1}\right)\right\}_{b=0}
$$

is (up to irrelevant normalizing constants) an equivariant Euler-Green current associated with the immersion of $E$ in $F$. So in fact, it is a well-understood object in a finite-dimensional context.

As pointed out in [BGS5, Theorem 3.15], if $G$ was instead a one-dimensional line bundle, then $\tilde{e}=\log |y|^{2}$. Now $G$ breaks down into a direct sum of vector bundles, which are the eigenspaces of $J$.

In [BGS5, Theorem 3.20], the behavior of Euler-Green currents under direct sums was studied. In particular, Euler-Green currents were shown to exhibit a transitivity property.

The transitivity property is expressed in a rather hidden form in our formula (6) in the Appendix for $C(s, x)$. In fact, note that for $k \in \mathbb{Z}^{*}$ and $x \in \mathbb{C}$

$$
\begin{gathered}
\int_{\mathbb{C}} 2 i k \pi \exp \left\{\left(-2 \pi^{2} k^{2}+i k \pi x\right)|Y|^{2}\right\} \exp \left(\frac{-u|Y|^{2}}{2}\right) \frac{d Y}{2 \pi} \\
=\frac{2 i k \pi}{4 \pi^{2} k^{2}-2 i k \pi x+u} .
\end{gathered}
$$

From formula (6) in the Appendix, we conclude that

$$
D(x)=-\int_{\mathbb{C}}\left(\sum_{k \in \mathbb{Z}}^{+\infty} 2 i k \pi \exp \left\{\left(-2 \pi^{2} k^{2}+i k \pi x\right)|Y|^{2}\right\}\right) \log |y|^{2} \frac{d Y}{2 \pi} .
$$

The term $\log |y|^{2}$ should be considered in (9.56) as the canonical one-dimensional Euler-Green current.

We also deduce from (9.55) that for $0<\operatorname{Re}(s)<\frac{1}{2}$

$$
C(s, x)=\int_{0}^{+\infty} r^{-s} \sum_{k \in \mathbb{Z}} 2 i k \pi \exp \left(\left(-4 \pi^{2} k^{2}+2 i k \pi x\right) r\right) d r
$$

From these considerations, we will obtain interesting formulas for $C(s, x)$ and $D(x)$.

Let $p_{t}(x)$ be the heat kernel on $S_{1}=\mathbb{R} / \mathbb{Z}$ associated with the operator $e^{t \Delta}$. Here $p_{t}(x)$ is calculated with respect to the Lebesgue measure on $S_{1}$. 
Theorem 9.13. The following identities holds:

$$
\begin{gathered}
C(s, x)=\int_{0}^{+\infty} r^{-s} \frac{\partial p_{r}}{\partial x}(r x) d r, \quad 0<\operatorname{Re}(s)<\frac{1}{2}, \\
D(x)=-\int_{0}^{+\infty}(\log r) \frac{\partial p_{r}}{\partial x}(r x) d r .
\end{gathered}
$$

Proof. Clearly,

$$
p_{t}(x)=\sum_{k \in \mathbb{Z}} e^{-4 \pi^{2} k^{2} t+2 i k \pi x} .
$$

The first line in (9.58) follows from (9.57). Since $D(x)=\frac{\partial C}{\partial s}(0, x)$, we get the second line in (9.58).

\section{APPENDIX \\ J.-M. Bismut AND C. Soulé}

The purpose of this appendix is to prove the following evaluation of the function $D(x)$ which was defined in $\S 8(\mathrm{~b})$. Let $\zeta(s)=\sum_{1}^{+\infty} 1 / n^{s}$ be the Riemann zeta function.

Theorem 1. For $x \in \mathbb{C},|x|<2 \pi$, the holomorphic function $D(x)$ is given by

$$
D(x)=\sum_{\substack{n \geq 1 \\ n \text { odd }}}\left\{\Gamma^{\prime}(1)+\sum_{j=1}^{n} \frac{1}{j}+\frac{2 \zeta^{\prime}(-n)}{\zeta(-n)}\right\} \zeta(-n) \frac{x^{n}}{n !} .
$$

Proof. The infinite product which defines $\varphi(u, x)$ in (6.4) is uniformly convergent on compact sets of $R^{+} \times \mathbb{C}$. We then deduce from (6.4) that, for $u \geq 0$ and $|x|<2 \pi$,

(2)

$$
\begin{aligned}
& \frac{-(\partial \varphi / \partial x)}{\varphi}(u, x) \\
& \quad=\sum_{k=1}^{+\infty} \frac{i}{2 k \pi}\left(\frac{1}{1-i x / 2 k \pi+u / 4 k^{2} \pi^{2}}-\frac{1}{1+i x / 2 k \pi+u / 4 k^{2} \pi^{2}}\right) .
\end{aligned}
$$

Equivalently, for $u \geq 0$ and $|x|<2 \pi$,

$$
\frac{-(\partial \varphi / \partial x)}{\varphi}(u, x)=-x \sum_{k=1}^{+\infty} \frac{1}{2 k^{2} \pi^{2}\left(\left(1+u / 4 k^{2} \pi^{2}\right)^{2}+x^{2} / 4 k^{2} \pi^{2}\right)} .
$$

By (7.67), we know that for $u \geq 1$

$$
\sum_{k=1}^{+\infty} \frac{k^{2}}{\left(k^{2}+u\right)^{2}} \leq C \sum_{k=1}^{+\infty} \frac{k^{2}}{k^{4}+u^{2}} \leq \frac{C^{\prime}}{\sqrt{u}} .
$$

Similarly, for $0 \leq u \leq 1$

$$
\sum_{1}^{+\infty} \frac{k^{2}}{\left(k^{2}+u\right)^{2}} \leq C^{\prime \prime}
$$


From (8.9), (4), (5), and the dominated convergence theorem, we deduce that for $s \in \mathbb{C}, 0<\operatorname{Re}(s)<\frac{1}{2}$, and $x \in \mathbb{C},|x|<2 \pi$,

$$
\begin{aligned}
C(s, x)=\sum_{k=1}^{+\infty} \frac{i}{2 k \pi} \frac{1}{\Gamma(s)} \int_{0}^{+\infty} u^{s-1}\left(\frac{1}{1-i x / 2 k \pi+u / 4 k^{2} \pi^{2}}\right. \\
\left.-\frac{1}{1+i x / 2 k \pi+u / 4 k^{2} \pi^{2}}\right) d u
\end{aligned}
$$

and that the convergence in the right-hand side of (6) is uniform on the compact subsets of the set of variation of $s$ and $x$.

Now if $a \in \mathbb{C}, \operatorname{Re}(a)>0$, and $s \in \mathbb{C}, 0<\operatorname{Re}(s)<1$, then $a^{s-1}$ is unambigously defined as a holomorphic function of $a$ and $s$ such that $1^{s-1}=$ 1. Then

$$
\begin{aligned}
& \frac{1}{\Gamma(s)} \int_{0}^{+\infty} \frac{u^{s-1} d u}{u+a}=\frac{a^{s-1}}{\Gamma(s)} \int_{0}^{+\infty} \frac{u^{s-1} d u}{u+1}, \\
& \frac{1}{\Gamma(s)} \int_{0}^{+\infty} \frac{u^{s-1} d u}{u+1}=\frac{1}{\Gamma(s)} \int_{R+\times R+} u^{s-1} e^{-t(u+1)} d u d t \\
& =\int_{0}^{+\infty} t^{-s} e^{-t} d t=\Gamma(1-s) \text {. }
\end{aligned}
$$

So from (6) and (7) we find that for $s \in \mathbb{C}, 0<\operatorname{Re}(s)<\frac{1}{2}$, and $x \in \mathbb{C}$, $|x|<2 \pi$,

$$
C(s, x)=i \Gamma(1-s) \sum_{k=1}^{+\infty}(2 k \pi)^{2 s-1}\left\{\left(1-\frac{i x}{2 k \pi}\right)^{s-1}-\left(1+\frac{i x}{2 k \pi}\right)^{s-1}\right\} .
$$

As $k \rightarrow+\infty$, for $|x|<2 \pi$

$$
\begin{aligned}
& (2 k \pi)^{2 s-1}\left(\left(1-\frac{i x}{2 k \pi}\right)^{s-1}-\left(1+\frac{i x}{2 k \pi}\right)^{s-1}\right) \\
& \simeq-2(s-1) i x(2 k \pi)^{2 s-2} .
\end{aligned}
$$

From (9), we deduce that the series in the right-hand side of (8) converges uniformly on the compact subsets of $\left\{s \in \mathbb{C}, \operatorname{Re}(s)<\frac{1}{2}\right\} \times\{x \in \mathbb{C},|x|<2 \pi\}$. The key fact is that $s=0$ is now included in the domain of convergence.

Since the series in (8) converges uniformly, and since the considered functions of $(s, x)$ are holomorphic on their domain of definition, we can differentiate term by term the series in the right-hand side of (8).

For $k \in \mathbb{N}^{*}$, set

$$
h_{k}(x)=i\left(\Gamma^{\prime}(1)-2 \log (2 k \pi)-\log \left(1+\frac{i x}{2 k \pi}\right)\right)\left(2 k \pi\left(1+\frac{i x}{2 k \pi}\right)\right)^{-1} .
$$

We then deduce from (8) that for $|x|<2 \pi$

$$
D(x)=\sum_{k=1}^{+\infty}\left(h_{k}(x)-h_{k}(-x)\right) \text {. }
$$


The series in (11) converges uniformly on the compact subsets of $\{x \in \mathbb{C},|x|<$ $2 \pi\}$. More precisely, one easily verifies that the series in the right-hand side of (11) converges normally on compact subsets of $\{x \in \mathbb{C},|x|<2 \pi\}$.

For any $k \in \mathbb{N}$, we can expand $h_{k}(x)$ in the form

$$
h_{k}(x)=\sum_{n=0}^{+\infty} a_{k}^{n} x^{n}, \quad|x|<2 \pi .
$$

By using Cauchy's integral formula, one verifies easily that for any $n \in \mathbb{N}, n$ odd

$$
\sum_{k=1}^{+\infty}\left|a_{k}^{n}\right|<+\infty
$$

and that

$$
D(x)=2 \sum_{\substack{n \geq 1 \\ n \text { odd }}}\left(\sum_{k=1}^{+\infty} a_{k}^{n}\right) x^{n} .
$$

Clearly, for $|x|<2 \pi$

$$
i\left(2 k \pi\left(1+\frac{i x}{2 k \pi}\right)\right)^{-1}=\frac{i}{2 k \pi} \sum_{n=0}^{+\infty}\left(\frac{-i x}{2 k \pi}\right)^{n} .
$$

By using the previous considerations and (15), we find that for $|x|<2 \pi$

$$
\begin{aligned}
\sum_{k=1}^{+\infty} i & \left\{\left(2 k \pi\left(1+\frac{i x}{2 k \pi}\right)\right)^{-1}-\left(2 k \pi\left(1-\frac{i x}{2 k \pi}\right)\right)^{-1}\right\} \\
& =\frac{1}{\pi} \sum_{n=1}^{+\infty}(-1)^{n-1} \zeta(2 n)\left(\frac{x}{2 \pi}\right)^{2 n-1} .
\end{aligned}
$$

Also for $\operatorname{Re}(s)>1$

$$
\zeta^{\prime}(s)=-\sum_{1}^{+\infty} k^{-s} \log (k) .
$$

From (15) and (17), we get for $|x|<2 \pi$

$$
\begin{gathered}
\sum_{k=1}^{+\infty} i \log k\left\{\left(2 k \pi\left(1+\frac{i x}{2 k \pi}\right)\right)^{-1}-\left(2 k \pi\left(1-\frac{i x}{2 k \pi}\right)\right)^{-1}\right\} \\
=-\frac{1}{\pi} \sum_{n=1}^{+\infty}(-1)^{n-1} \zeta^{\prime}(2 n)\left(\frac{x}{2 \pi}\right)^{2 n-1} .
\end{gathered}
$$

Also

$$
\begin{aligned}
& i \log \left(1+\frac{i x}{2 k \pi}\right)\left(2 k \pi\left(1+\frac{i x}{2 k \pi}\right)\right)^{-1} \\
& =-\frac{i}{2 k \pi} \sum_{n=1}^{+\infty} \frac{1}{n}\left(\frac{-i x}{2 k \pi}\right)^{n} \sum_{n^{\prime}=0}^{+\infty}\left(\frac{-i x}{2 k \pi}\right)^{n^{\prime}} .
\end{aligned}
$$


From (19) and the previous considerations, we find that for $|x|<2 \pi$

$$
\begin{aligned}
\sum_{k=1}^{+\infty}\{ & i \log \left(1+\frac{i x}{2 k \pi}\right)\left(2 k \pi\left(1+\frac{i x}{2 k \pi}\right)\right)^{-1} \\
& \left.-i \log \left(1-\frac{i x}{2 k \pi}\right)\left(2 k \pi\left(1-\frac{i x}{2 k \pi}\right)\right)^{-1}\right\} \\
= & -\frac{1}{\pi} \sum_{n=1}^{+\infty}(-1)^{n-1}\left(\sum_{j=1}^{2 n-1} \frac{1}{j}\right) \zeta(2 n)\left(\frac{x}{2 \pi}\right)^{2 n-1} .
\end{aligned}
$$

From (10), (11), (14), (16), and (20), we get for $|x|<2 \pi$

$$
\begin{aligned}
D(x)=\frac{1}{\pi} \sum_{n=1}^{+\infty} & \left(\Gamma^{\prime}(1)-2 \log (2 \pi)+\sum_{j=1}^{2 n-1} \frac{1}{j}+\frac{2 \zeta^{\prime}(2 n)}{\zeta(2 n)}\right) \\
& \times(-1)^{n-1} \zeta(2 n)\left(\frac{x}{2 \pi}\right)^{2 n-1} .
\end{aligned}
$$

Now $\zeta(s)$ verifies the functional equation

$$
\pi^{-s / 2} \Gamma(s / 2) \zeta(s)=\pi^{-(1-s) / 2} \Gamma((1-s) / 2) \zeta(1-s) .
$$

By taking logarithmic derivatives in (22), we get

$$
\begin{aligned}
& -\frac{1}{2} \log \pi+\frac{1}{2} \frac{\Gamma^{\prime}(s / 2)}{\Gamma(s / 2)}+\frac{\zeta^{\prime}(s)}{\zeta(s)} \\
& \quad=\frac{1}{2} \log \pi-\frac{1}{2} \frac{\Gamma^{\prime}((1-s) / 2)}{\Gamma((1-s) / 2)}-\frac{\zeta^{\prime}(1-s)}{\zeta(1-s)} .
\end{aligned}
$$

Also, by [NO, p. 21], we know that for $n \in \mathbb{N}$

$$
\begin{gathered}
\frac{\Gamma^{\prime}(n)}{\Gamma(n)}=\Gamma^{\prime}(1)+\sum_{j=1}^{n-1} \frac{1}{j}, \\
\frac{\Gamma^{\prime}((1-2 n) / 2)}{\Gamma((1-2 n) / 2)}=\Gamma^{\prime}(1)-2 \log 2+2 \sum_{j=1}^{n} \frac{1}{2 j-1} .
\end{gathered}
$$

From (23) and (24), we easily deduce that

$$
\begin{aligned}
\Gamma^{\prime}(1) & -2 \log (2 \pi)+\sum_{j=1}^{2 n-1} \frac{1}{j}+\frac{2 \zeta^{\prime}(2 n)}{\zeta(2 n)} \\
= & -\left(\Gamma^{\prime}(1)+\sum_{j=1}^{2 n-1} \frac{1}{j}+\frac{2 \zeta^{\prime}(1-2 n)}{\zeta(1-2 n)}\right) .
\end{aligned}
$$

Also by [S, pp. 117, 148], we know that

$$
\zeta(2 n)=\frac{2^{2 n-1}}{(2 n-1) !}(-1)^{n} \pi^{2 n} \zeta(1-2 n) .
$$

From (21), (25), and (26), we get (1). $\square$ 


\section{REFERENCES}

[A] M. F. Atiyah, Circular symmetry and stationary phase approximation, Proc. Conf. in honor of L. Schwartz, Astérisque 131 (1985), 43-59.

[AB] M. F. Atiyah and R. Bott, A Lefschetz fixed point formula for elliptic complexes. I, Ann. of Math. (2) 86 (1967), 374-407; II, Ann. of Math. (2) 88 (1968), 451-491.

[ABP] M. F. Atiyah, R. Bott, and V. K. Patodi, On the heat equation and the Index Theorem, Invent. Math. 19 (1973), 279-330.

[ABS] M. F. Atiyah, R. Bott, and A. Shapiro, Clifford modules, Topology 3 (1964), 3-38.

[AHS] M. F. Atiyah, N. J. Hitchin, and I. M. Singer, Self-duality in four dimensional Riemannian geometry, Proc. Roy. Soc. London Ser. A 362 (1978), 425-461.

[BaFM] P. Baum, W. Fulton, and R. Macpherson, Riemannian-Roch for singular varieties, Inst. Hautes Études Sci. Publ. Math. 45 (1975), 101-146.

[BeV] N. Berline and M. Vergne, A computation of the equivariant index of the Dirac operator, Bull. Soc. Math. France 113 (1985), 305-345.

[B1] J.-M. Bismut, The Index Theorem for families of Dirac operators: two heat equation proofs, Invent. Math. 83 (1986), 91-151.

[B2] _ Localization formulas, superconnections, and the Index Theorem for families, Comm. Math. Phys. 103 (1986), 127-166.

[B3] __ Equivariant Bott-Chern currents and the Ray-Singer analytic torsion, Math. Ann. (to appear).

[B4] _ The Witten complex and the degenerate Morse inequalities, J. Differential Geom. 23 (1986), 207-240.

[B5] __ Superconnection currents and complex immersions, Invent. Math. (to appear).

[B6] __, Transformations différentiables du mouvement Brownien, Proc. Conf. in honor of L. Schwartz, Astérisque 131 (1985), 61-87.

[B7] _ Martingales, the Malliavin calculus and hypoellipticity under general Hörmander's conditions, Z. Wahrsch. Verw. Gebiete 56 (1981), 469-505.

[B8] _- The Atiyah-Singer Theorems: A probabilistic approach. I, J. Funct. Anal. 57 (1984), 56-99.

[B9] _ Index Theorem and equivariant cohomology on the loop space, Comm. Math. Phys. 98 (1985), 213-237.

[B10] __ Large deviations and the Malliavin calculus, Progress in Math., no. 45, Birkhäuser, Basel, Boston, and Stuttgart, 1984.

[B11] __, The infinitesimal Lefschetz formulas: A heat equation proof, J. Funct. Anal. 62 (1985), 435-457.

[B12] __ Complexe de Koszul, oscillateur harmonique et classe de Todd, C. R. Acad. Sci. Paris Sér. I Math. 309 (1989), 111-114.

[BGS1] J-M. Bismut, H. Gillet, and C. Soulé, Analytic torsion and holomorphic determinant bundles. I, Comm. Math. Phys. 115 (1988), 49-78.

[BGS2] _ Analytic torsion and holomorphic determinant bundles. II, Comm. Math. Phys. 115 (1988), 79-126.

[BGS3] __, Analytic torsion and holomorphic determinant bundles. III, Comm. Math. Phys. 115 (1988), 301-351.

[BGS4] __ Bott-Chern currents and complex immersions, Duke Math. J. (to appear).

[BGS5] _ Complex immersions and Arakelov geometry, Inst. Hautes Études Sci., preprint $\mathrm{M} / 88 / 64$.

[BL1] J.-M. Bismut and G. Lebeau, Immersions complexes et métriques de Quillen, C. R. Acad. Sci. Paris Sér. I Math. 309 (1989), 111-114.

[BL2] _ Complex immersions and Quillen metrics (to appear). 
[BoC] R. Bott and S. S. Chern, Hermitian vector bundles and the equidistribution of the zeros of their holomorphic sections, Acta Math. 114 (1968), 71-112.

[D] S. Donaldson, Anti-self-dual Yang-Mills connections over complex algebraic surfaces and stable vector bundles, Proc. London Math. Soc. 50 (1985), 1-26.

[DeM] C. Dellacherie and P.-A. Meyer, Probabilités et potentiels, Chapitre V à VIII. Théorie des martingales, Hermann, Paris, 1980.

[Ge] E. Getzler, A short proof of the Atiyah-Singer Index Theorem, Topology 25 (1986), 111-117.

[GH] P. Griffiths and J. Harris, Principles of algebraic geometry, Wiley, New York, 1978.

[GS] H. Gillet and C. Soulé, Analytic torsion and the arithmetic Todd genus, Topology (to appear).

[GIJ] J. Glimm and A. Jaffe, Quantum physics, Springer, Berlin, Heidelberg, and New York, 1987.

[H] N. Hitchin, Harmonic spinors, Adv. in Math. 14 (1974), 1-55.

[IkW] N. Ikeda and S. Watanabe, Stochastic differential equations and diffusion processes, NorthHolland, Amsterdam, 1981.

[IMK] K. Ito and H. McKean, Diffusion processes and their sample paths, Grundl. Math. Wiss., bd. 125, Springer, Berlin, Heidelberg, and New York, 1974.

[IN] K. Ito and M. Nisio, On the convergence of sums of independent Banach space valued random variables, Osaka J. Math. 5 (1968), 35-48.

[KM] F. F. Knudsen and D. Mumford, The projectivity of the moduli space of stable curves, I: Preliminaries on "det" and "div", Math. Scand. 39 (1976), 19-55.

[Le] P. Levy, Wiener's random functions, and other Laplacian random functions, Proc. Second Berkeley Symposium on Mathematical Statistics and Probability (J. Neyman, ed.), Univ. of California Press, Berkeley, CA, 1951, pp. 171-187.

[Ma] P. Malliavin, Stochastic calculus of variations and hypoelliptic operators, Proc. Conf. on Stochastic Differential Equations, Kyoto, 1976, Wiley, New York, 1978, pp. 195-263.

[MQ] V. Mathai and D. Quillen, Superconnections, Thom classes and equivariant differential forms, Topology 25 (1986), 85-110.

[NO] A. Nikiforov and V. Ouvarov, Eléments de la théorie des fonctions spéciales, Mir, Moscow, 1976.

[Q1] D. Quillen, Superconnections and the Chern character, Topology 24 (1985), 89-95.

[Q2] __ Determinants of Cauchy-Riemann operators over a Riemann surface, Funct. Anal. Appl. 14 (1985), 31-34.

[RS] D. B. Ray and I. M. Singer, Analytic torsion for complex manifolds, Ann. of Math. (2) 98 (1973), 154-177.

[S] J.-P. Serre, Cours d'arithmétique, Presses Universitaires de France, Paris, 1970.

[Si1] B. Simon, Functional integration and quantum physics, Academic Press, New York, 1979.

[Si2] _ Notes on infinite determinants of Hilbert space operators, Adv. in Math. 24 (1977), 244-273.

[W] E. Witten, Supersymmetry and Morse theory, J. Differential Geom. 17 (1982), 661-692.

[Y] M. Yor, Remarques sur une formule de P. Lévy, Séminaire de Probabilités, no. XIV, Lecture Notes in Math., vol. 784, Springer, Berlin, Heidelberg, and New York, 1980, pp. 343-346.

ABSTRACT. In this paper, we construct secondary characteristic classes associated with a short exact sequence of holomorphic Hermitian vector bundles. These secondary invariants are generalized analytic torsion forms associated with a family of elliptic operators. They are calculated in terms of Bott-Chern forms and of a certain complicated characteristic class. In a joint calculation with C. Soulé, we relate this characteristic class to the arithmetic Todd genus of Gillet and Soulé.

Defartement de Mathematique, Université de Paris-Sud, BÂtiment 425, 91405 Orsay, FRANCE 